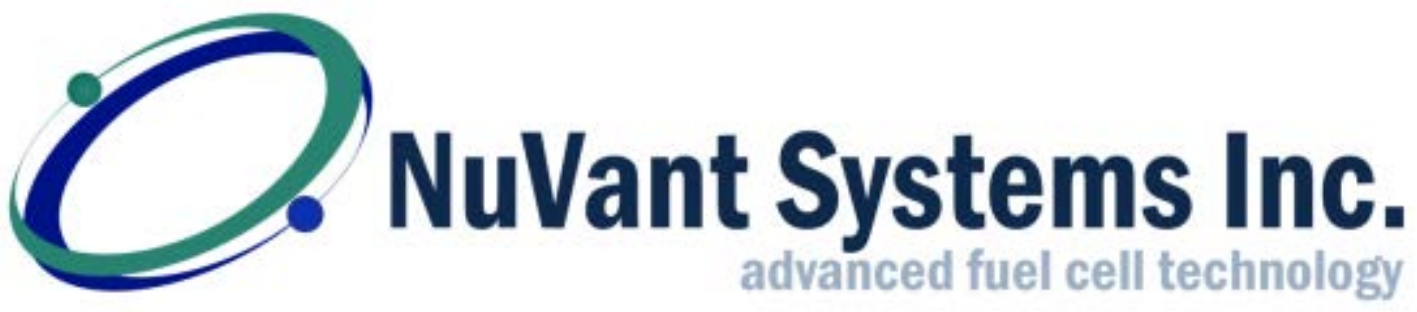

AWARD NUMBER

DEFC2609FE0000982

SUBMITTED BY

NuVant Systems, Inc.

130 N West Street

Crown Point, IN 46307

PROJECT TITLE

Improved Flow-Field Structures for Direct Methanol Fuel Cells

\title{
PRINCIPAL INVESTIGATOR
}

Bogdan Gurau

Phone: 219-644-3232

b.gurau@nuvant.com

SUBMITTED TO

U. S. Department of Energy

National Energy Technology Laboratory

DATE SUBMITTED

10/10/2013

(Final Technical Report) 


\section{DISCLAIMER}

This report was prepared as an account of work sponsored by an agency of the United States Government. Neither the United States Government nor any agency thereof, nor any of their employees, makes any warranty, express or implied, or assumes any legal liability or responsibility for the accuracy, completeness, or usefulness of any information, apparatus, product, or process disclosed, or represents that its use would not infringe privately owned rights. Reference herein to any specific commercial product, process, or service by trade name, trademark, manufacturer, or otherwise does not necessarily constitute or imply its endorsement, recommendation, or favoring by the United States Government or any agency thereof. The views and opinions of authors expressed herein do not necessarily state or reflect those of the United States Government or any agency thereof. 


\section{Table of Contents}

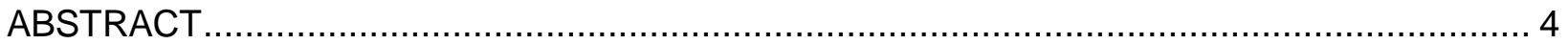

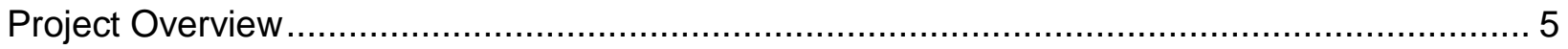

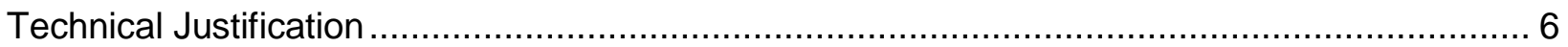

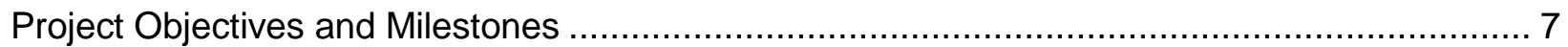

Milestone\#1. Produce Fluent models for various flow-field geometries with various porosities... 9

Milestone\#2. Fabricate porous plates according to model findings ..................................25

Milestone\#3. Maximize fuel energy density by optimization of MEA structure and operating scheme \& perform extended life tests of optimized MEAs on single cells ............................33

Milestone\#4. Build a research-grade short stack ........................................................45

Milestone\#5. Perform additional modeling to account for transport of species within the cell and

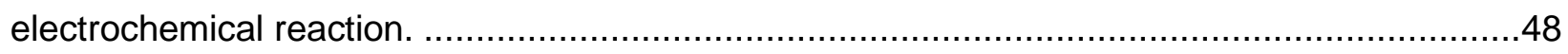

Milestone\#6: Manufacture gas diffusion layers with user defined levels of hydrophobicity. .......66

Milestone\#7: Optimize the cathode flow field design in order to accomplish uniform flow throughout the entire electrode area. ........................................................................ 74

Milestone\#8: Step Impedance Spectroscopy. Design and operation. ................................99

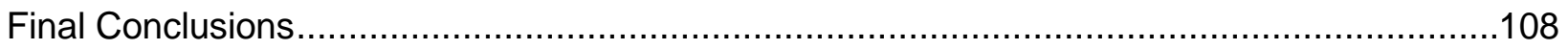




\section{ABSTRACT}

The direct methanol fuel cell (DMFC) is ideal if high energy-density liquid fuels are required. Liquid fuels have advantages over compressed hydrogen including higher energy density and ease of handling. Although state-of-the-art DMFCs exhibit manageable degradation rates, excessive fuel crossover diminishes system energy and power density. Although use of dilute methanol mitigates crossover, the concomitant lowering of the gross fuel energy density (GFED) demands a complex balance-of-plant (BOP) that includes higher flow rates, external exhaust recirculation, etc. An alternative approach is redesign of the fuel delivery system to accommodate concentrated methanol.

NuVant Systems Inc. (NuVant) will maximize the GFED by design and assembly of a DMFC that uses near neat methanol. The approach is to tune the diffusion of highly concentrated methanol (to the anode catalytic layer) to the back-diffusion of water formed at the cathode (i.e. in situ generation of dilute methanol at the anode layer). Crossover will be minimized without compromising the GFED by innovative integration of the anode flow-field and the diffusion layer. The integrated flow-field-diffusion-layers (IFDLs) will widen the current and potential DMFC operating ranges and enable the use of cathodes optimized for hydrogen-air fuel cells. 


\section{Project Overview}

Direct liquid fuel oxidation fuel cells hold great promise for portable power sources and even intermediate power sources (low hundreds of watts) because the fuels have high energy density. One of the fuels with high energy density is methanol, so the direct methanol fuel cell (DMFC) is perfectly suitable for the above mentioned applications.

Currently, state-of-the-art DMFCs have acceptable degradation rates but the excessive crossover of the fuel greatly diminishes the ability to harvest the energy contained in the fuel. The crossover impediment can be mitigated by the use of diluted fuel but this heavily decreases the gross fuel energy density (GFED) and brings about a complex and bulky balance of plant, which impacts the final energy and power density of the system. If DMFCs are going to be successful in coming years an alternative approach, which enables the use of concentrated fuel, will have to be devised.

NuVant Systems, Inc. has tackled the problem of methanol crossover by employing highly porous structures at the DMFC anode which allowed for controlled delivery of highly concentrated methanol to the anode side. The methanol delivery to the anode side was adjusted in concert with the back-diffusion of water from the cathode side such that diluted methanol was created in-situ at the anode. In this way crossover was minimized and high GFED was achieved.

The success resides in the tightly controlled structure of the porous material and the fuel delivery mode. The pore diameter and total porosity play a very important role on how and how much of the highly concentrated methanol arrives at the anode.

In order to best design these porous structures, a thorough understanding of flow and diffusion through porous media is required. By employing computational fluid dynamics (CFD) modeling NuVant studied various porosities, various geometries and various flow fields which enabled optimal delivery of methanol to the reaction site.

Based on the optimal design of these structures NuVant built a short, research-grade DMFC stack that was operated to demonstrate that the concept can be extended to more than one cell. 


\section{Technical Justification}

The best way to mitigate (or alleviate) the methanol crossover problem is to judiciously design the manner in which methanol is brought to the reaction site so that little or no methanol makes its way to the cathode.

The redesign of the anode fuel delivery system, such that highly concentrated methanol entering the anode compartment is almost entirely consumed within the anode catalyst layer with minimal flux through the Nafion membrane is a relatively new concept. It has the main following advantages:

- Increase in the fuel utilization

- Increase in electrical performance

- Simple and small balance of plant

The best way to point out how this new concept alleviates some of the problems associated with DMFCs is to look at how GFED is calculated.

$$
\mathrm{W}_{\mathrm{L}}=\mathrm{c}_{\mathrm{M}} \times \mathrm{E}_{\text {cell }} \times \% \mathrm{U} \times \mathrm{n} \times \mathrm{F} \times \frac{1}{3600}
$$

where:

$\mathrm{W}_{\mathrm{L}}=$ volumetric GFED $\left(\mathrm{W} * \mathrm{hr} / \mathrm{L}_{\text {fuel }}\right)$

$\mathrm{C}_{\mathrm{M}}=$ fuel concentration (moles/L)

$\mathrm{E}_{\text {cell }}=$ operating cell voltage $(\mathrm{V})$

$\% U$ = fuel utilization (between 0 -1)

$\mathrm{n}=$ number of electrons generated in the reaction (6 equiv/mole)

$\mathrm{F}=$ Faraday's constant (96485 C/equiv)

The process of methanol oxidation always involves 6 electrons so " $n$ " remains fixed. Faraday's constant is a fixed universal constant (96485 C/equiv). However the first 3 terms in equation (1) play an overwhelming role on what the GFED ends up being.

If water can be returned by back-diffusion from the cathode it means that highly concentrated methanol can be delivered to the anode because the dilution will be created insitu. This increases the value of " $\mathrm{C}_{\mathrm{M}}$ " (neat methanol is ca. $24.7 \mathrm{moles} / \mathrm{L}$ ).

If the methanol is consumed almost entirely within the anode (i.e. no more methanol to

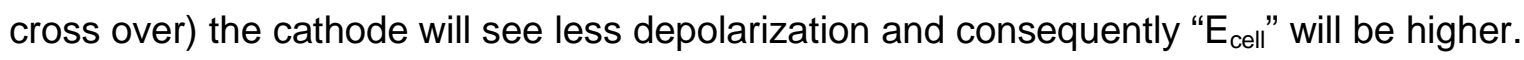

By the same token, if methanol is consumed at the anode (i.e. faradaicaly efficient) the fuel utilization "\%U" will go up. 
If " $\mathrm{C}_{\mathrm{M}}$ ", " $\mathrm{E}_{\text {cell }}$ " and "\%U" increase the GFED ("W $\mathrm{W}_{\mathrm{L}}$ ") increases. For example, operating with $24 \mathrm{M}$ methanol (almost neat) at 0.4V with $90 \%$ utilization the GFED would be:

$$
\mathrm{W}_{\mathrm{L}}=24 \cdot \frac{\text { mole }}{\mathrm{L}_{\text {fuel }}} \times 0.4 \cdot \mathrm{V} \times 0.9 \times 6 \cdot \frac{\text { equiv }}{\text { mole }} \times 96485 \cdot \frac{\mathrm{C}}{\text { equiv }} \times \frac{1}{3600}=1389 \frac{\mathrm{W} \cdot \mathrm{hr}}{\mathrm{L}_{\text {fuel }}}
$$

This will require optimized design of the cathode structure and use of thin membranes to facilitate transport of water from the cathode back to the anode. The local dilution of methanol at the anode catalytic layer would provide the water required for the 6-electron methanol electrooxidation process. The back-diffusion of water will allow use of highly concentrated methanol, thus increasing the GFED.

This strategy will simplify the balance of plant and increase the system energy density $\left(\mathrm{W} * \mathrm{hr} / \mathrm{L}_{\text {system }}\right)$.

\section{Project Objectives and Milestones}

The project objectives are as follows:

1) Model the transport of methanol through various porous structures (different pore size distribution and different total porosity) and obtain optimal porous structures. This task is designated to provide a series of models that simulate the lateral and through plate flow of the incoming fuel. Uniform lateral distribution and controlled flow through the porous plate is of utmost importance for controlling the rate at which the fuel arrives at the fuel cell anode. This will ultimately decide the fuel utilization and GFED.

2) Fabricate porous plates based on the modeling results and prove that concentrated methanol can be used to run a single cell DMFC at high GFED and high power density. For the accomplishment of this task the modeling results are put into practice. Porous plates are fabricated according to the modeling results i.e. with the desired porosity and permeability and the optimum flow-fields are engraved into them according to the models that revealed the best lateral fuel distribution. These plates are integrated into research-grade fuel cells and the operation of the resulting DMFCs is optimized for various conditions (operating current, temperature, fuel flow, etc.) 
3) Build a short research-grade DMFC stack prototype to prove that the concept of controlling the methanol concentration using porous structures and water back-diffusion from the cathode is feasible.

While the above three tasks were the only ones that were originally proposed, a number of additional tasks were deemed to be necessary and were introduced via a no cost extension. They were as follows:

4) Model the transport and electrochemical reaction through the entire fuel cell and verify whether the model accurately matches the fuel cell output. The successful operation of the IFDL-based cell is fully dependent on in-situ water return from the cathode to the anode. One mole of water is needed for each mole of $\mathrm{MeOH}$ in the anodic reaction. Additional modeling is needed to account for the water production at the cathode and the anodic water loss by electro-osmotic drag.

5) Fabricate super-hydrophobic cathodic gas diffusion layers in order to block water loss from the cathode and promote water back-diffusion to the anode. Without electrically conductive super-hydrophobic materials at the cathode of the IFDL cell, most of the water produced cathodically would be transported out and away from the cell. The successful in-situ dilution of the methanol entering the anode is dependent on the ability of the cathodic super-hydrophobic material to prevent the water loss.

6) Cathode flow field modeling and design to improve the uniformity of distribution of air flow to the cathode. Poor fuel flow (i.e. non-uniform) characteristics within flow field leads to incomplete chemical reaction. The proposed solution was to build a device that enabled NuVant to manipulate the flow at inlet/outlet as to optimize the flowfield.

7) Step impedance spectroscopy to determine the value of electrolyte and MEA impedance.

In order to assign metrics to assess the level of accomplishment of these tasks, a number of milestones were devised as follows:

Milestone\#1: Produce Fluent models for various flow-field geometries with various porosities

- Milestone\#2: Fabricate porous plates according to model findings

- Milestone\#3: Maximize fuel energy density by optimization of MEA structure and operating scheme \& perform extended life tests of optimized MEAs

u Milestone\#4: Build a research-grade short stack 
Milestone\#5: Perform additional modeling to account for transport of species within the cell and electrochemical reaction.

Milestone\#6: Manufacture gas diffusion layers with user defined levels of hydrophobicity

- Milestone\#7: Optimize the cathode flow field design in order to accomplish uniform flow throughout the entire electrode area

口 Milestone\#8: Step Impedance Spectroscopy. Design and operation

\section{Milestone\#1. Produce Fluent models for various flow-field geometries with various porosities}

Modeling flow through porous media is usually done by employing various forms of Darcy's law. Darcy's law correlates the discharge of fluid through porous media with the permeability of the porous substrate, the pressure gradient applied and the fluid physical properties (e.g. viscosity). In its simplest form, Darcy's law is expressed by the following equation:

$$
\mathrm{Q}=\frac{-\mathrm{k} \cdot \mathrm{A}}{\mu} \cdot \frac{\Delta \mathrm{p}}{\delta}
$$

where:

$\mathrm{Q}=$ volumetric flowrate $\left(\mathrm{m}^{3} / \mathrm{sec}\right)$

$\mathrm{k}=$ permeability $\left(\mathrm{m}^{2}\right)$

$A=$ cross-sectional area $\left(\mathrm{m}^{2}\right)$

$\Delta \mathrm{p}=$ pressure difference on opposite sides of diffusion path $(\mathrm{Pa})$

$\mu=$ dynamic viscosity (Pa*sec)

$\delta=$ length over which the pressure drop occurs $(\mathrm{m})$

A diagram illustrating the porous plate with all the definitions involved in Darcy's law is shown in the following figure: 


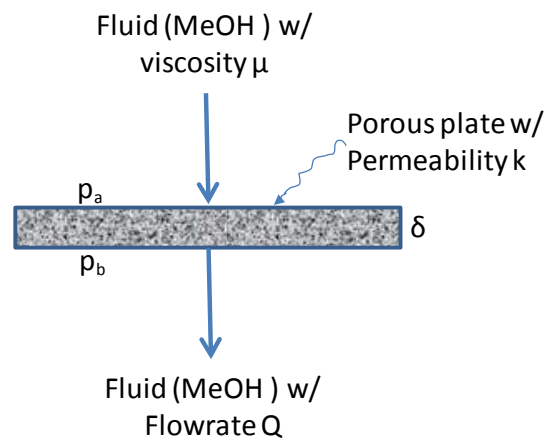

Figure 1. Illustration of parameters contained in Darcy's law

The permeability "k" in Darcy's law can be expressed by the following equation:

$$
k=C \cdot d^{2}
$$

where:

$\mathrm{k}=$ permeability $\left(\mathrm{m}^{2}\right)$

$\mathrm{C}=$ dimensionless constant that is related to the configuration of the flow-paths (tortuosity)

$\mathrm{d}=$ average or effective pore diameter $(\mathrm{m})$

The important part was to be able to tweak the porous material permeability (i.e. pore characteristics - pore diameter and total porosity) and the pressure gradient (i.e. $\Delta p / \delta$ ) such that a uniform stream of methanol emerged from the porous plate at a flowrate $\mathrm{Q}$ to be delivered to the reaction site.

For a DMFC operating at a constant current "I" the fuel required to sustain the current "I" will have to arrive at the reaction site (the anode) at a rate of:

$$
\mathrm{Q}=\frac{\mathrm{I}}{\mathrm{n} \cdot \mathrm{F}} \cdot \frac{\mathrm{M}}{\rho} \cdot 60
$$

where:

$\mathrm{Q}=$ volumetric flowrate of methanol ( $\mathrm{mL} / \mathrm{min})$

$\mathrm{n}=$ number of electrons generated in the reaction (6 equiv/mole)

$\mathrm{F}=$ Faraday's constant (96485 Clequiv)

$\mathrm{M}=$ molecular weight of methanol (32 $\mathrm{gm} / \mathrm{mole})$

$\rho=$ methanol density $(0.7918 \mathrm{gm} / \mathrm{mL})$ 
In principle, we attempted to correlate (match) the rate at which methanol emerged from the porous plate with the rate at which methanol was consumed in the electrochemical reaction as shown in equation 5 . In reality a small quantity of methanol will cross over and also a small amount will be engaged by the $\mathrm{CO}_{2}$ gas resulting from the methanol electro-oxidation and carried outside as anode exhaust. This means that the flowrate $\mathrm{Q}$ emerging from the porous plate had to be slightly larger than the amount of methanol required by the current "I".

The first geometry that we proposed is shown in figure 2 and figure 3.

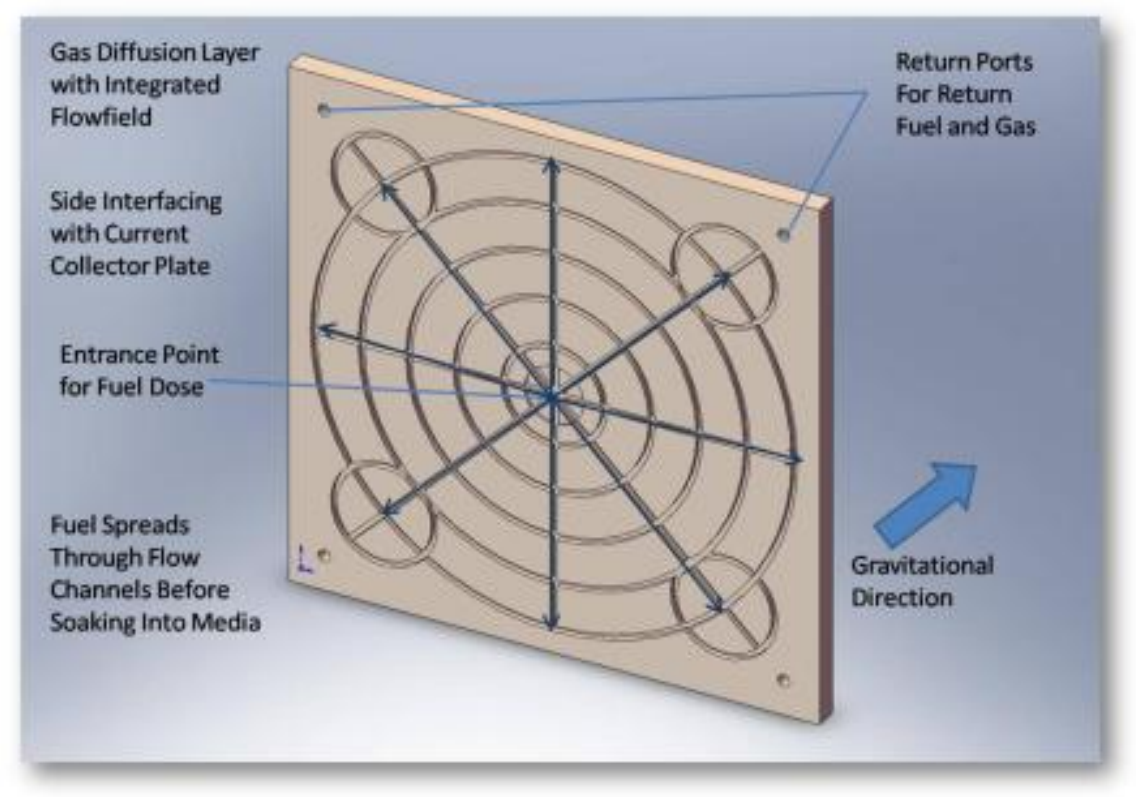

Figure 2. The fuel side of the Integrated Flow field Difussion Layer (IFDL)

The idea is that fuel is pumped through and entry point in the middle of the IFDL from where it will spread out laterally, as shown in figure 2, occupying as much as possible of the area of the electrode before it soaks into the material. Once it soaks into the material, it crosses it at some rate which is dependent on the porous structure of the plate.

The opposite of the side shown in figure 2 is not flat and has its own flow field that enables gas evacuation. It is shown in figure 3 . 


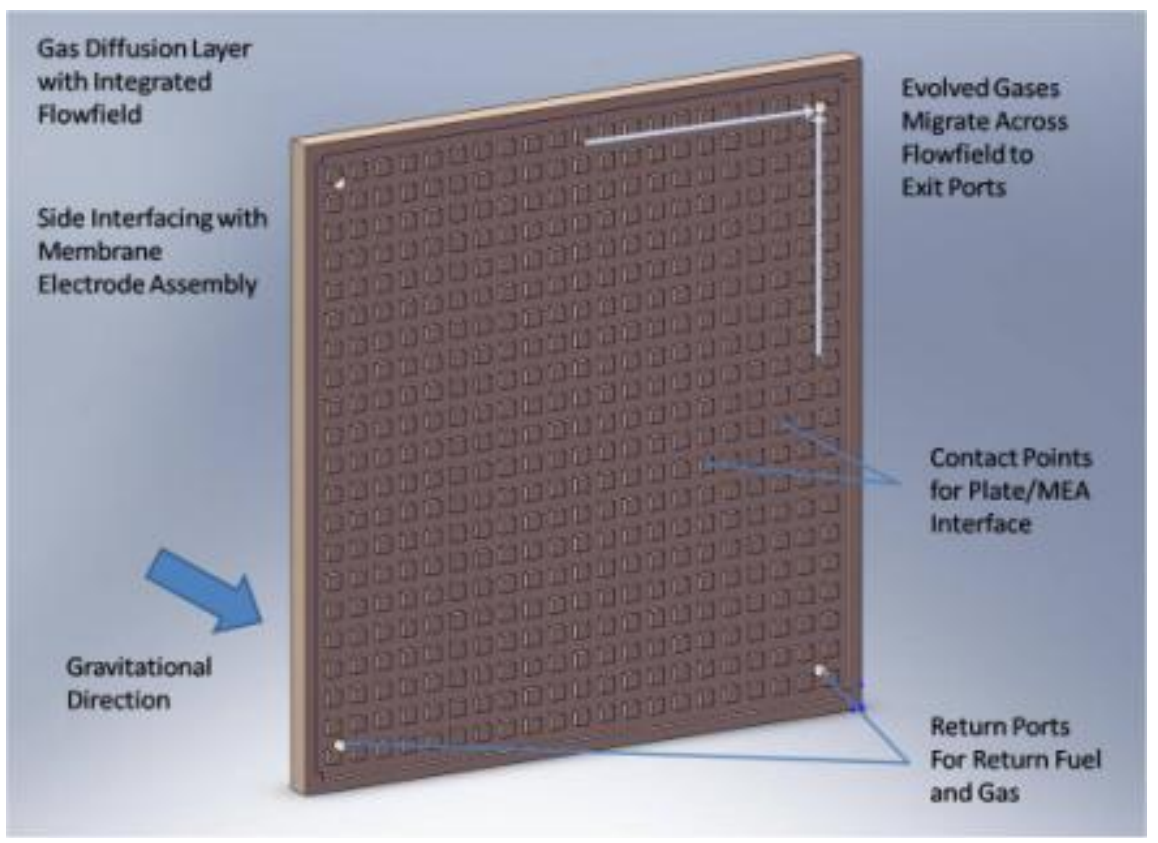

Figure 3. The gas evacuation side of the IFDL

CO2 is evolved in the anodic reaction and needs to be evacuated so that is why the back of the IFDL had its own flowfield, shown in figure 3 . The gas will find its way towards the corners of the plate where it will be vented through the 4 holes, also shown in figure 3 . This side will be interfaced with the fuel cell membrane electrode assembly. The lands on this face will be the contact points that will provide the electrical contact between the membrane electrode assembly and the current collectors on the outside.

\section{Boundary Conditions}

In order to commence any modeling some boundary conditions need to be set. The data that is required is the following:

- Inlet flowrate: $0.016 \mathrm{gm} / \mathrm{min}$ of $\mathrm{MeOH}$ solution at whatever concentration

- Inlet pressure: 1 psi above atmospheric pressure (1 psig)

- Inlet temperature: $60^{\circ} \mathrm{C}$

- $\quad$ Average pore diameter: 10 microns

- $\quad$ Total porosity: $40 \%$

In order to characterize the flow through the porous media one needs to know the value of the viscous resistance. The viscous resistance is the inverse of the permeability. For a low flowrate like the one used in our modeling, the regime is very laminar and the permeability can be calculated using the well known Kozeny-Carman relationship: 


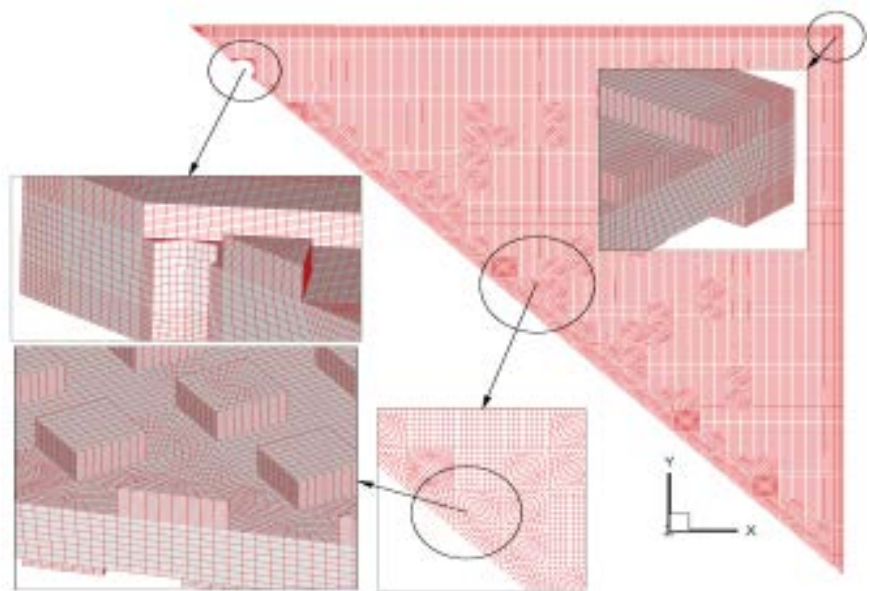

where:

$\mathrm{Dp}=$ average pore diameter $(\mathrm{m})$

$\varepsilon=$ total porosity

The modeling was performed using "Fluent" but before the flow model can be run the proper meshing of the geometry is accomplished using "iSim".

The meshing of the system is shown in figure 4:

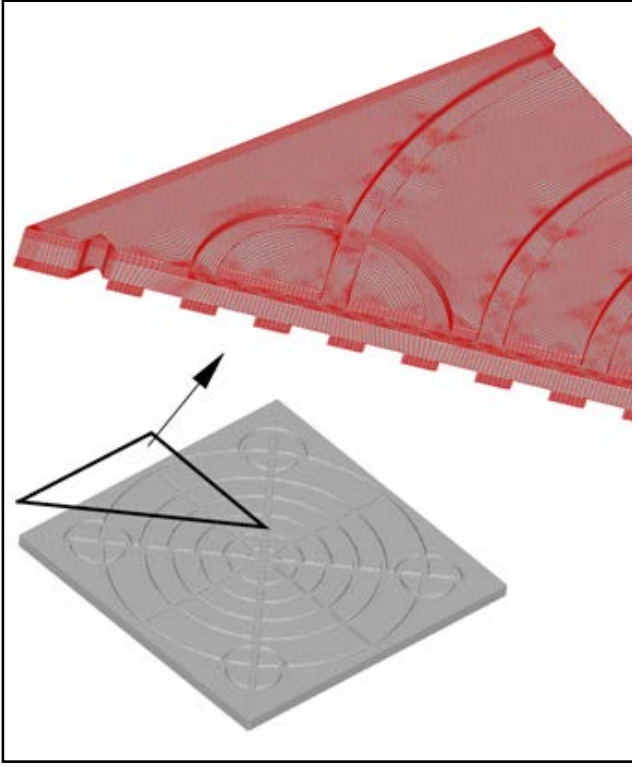

Figure 4: The meshing of IFDL plate $\quad \alpha=\frac{D_{p}^{2}}{150} \frac{\varepsilon^{3}}{(1-\varepsilon)^{2}}$

It can be noticed from figure 4 that, for simplicity, only $1 / 8$ th of the plate needs to be subjected to the modeling as there is perfect symmetry around.

Detailed images of the meshing are shown in figure 5: 


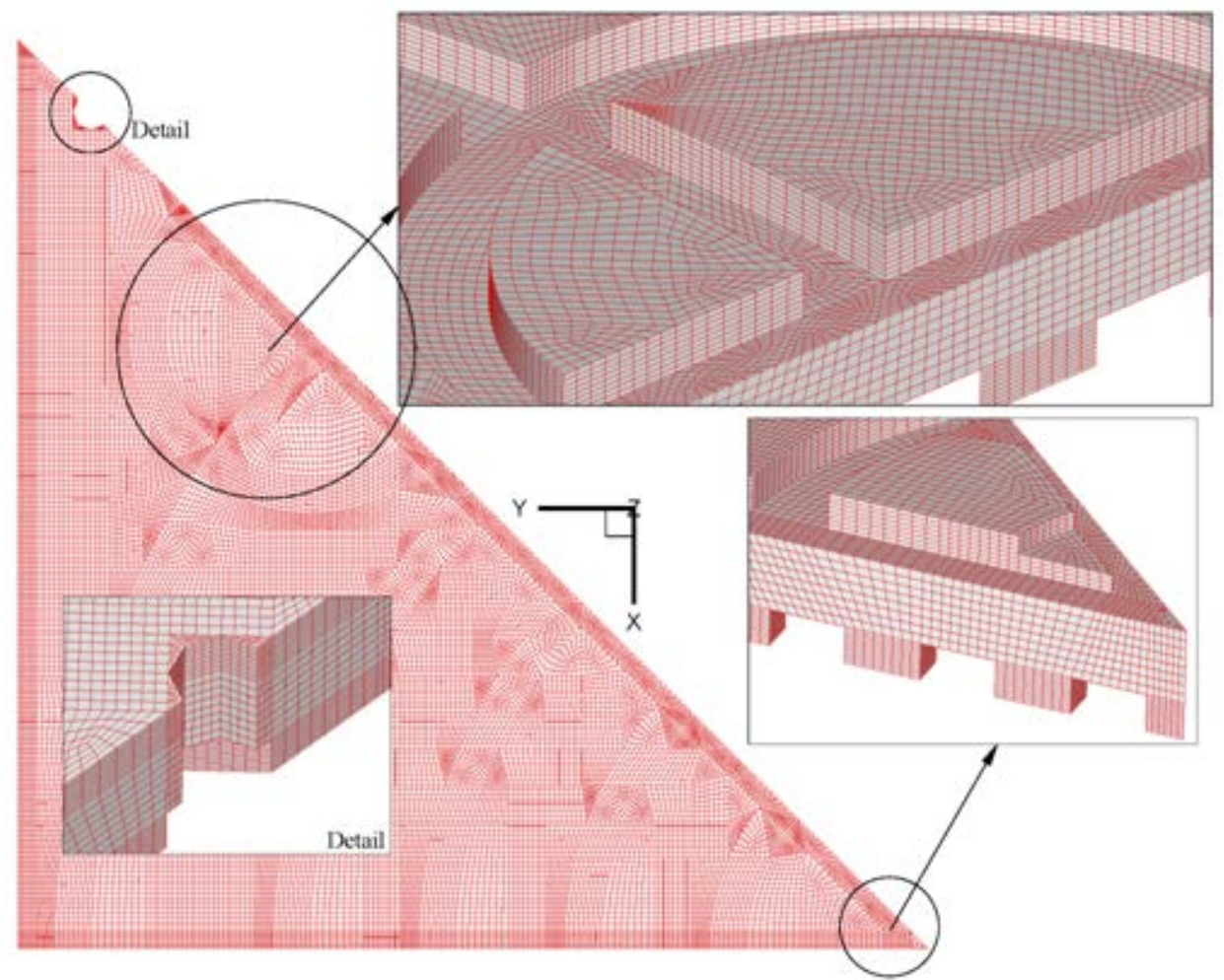

Figure 5: Detailed images of meshing various features of the IFDL plate

The calculations are carried out using the finite element method and their purpose is to determine how uniform is the spreading of the liquid on the liquid side of the IFDL plate as well as what is the liquid discharge rate at the other end of the plate as a function of porosity and average pore diameter.

The pressure field is shown in figure 6. 


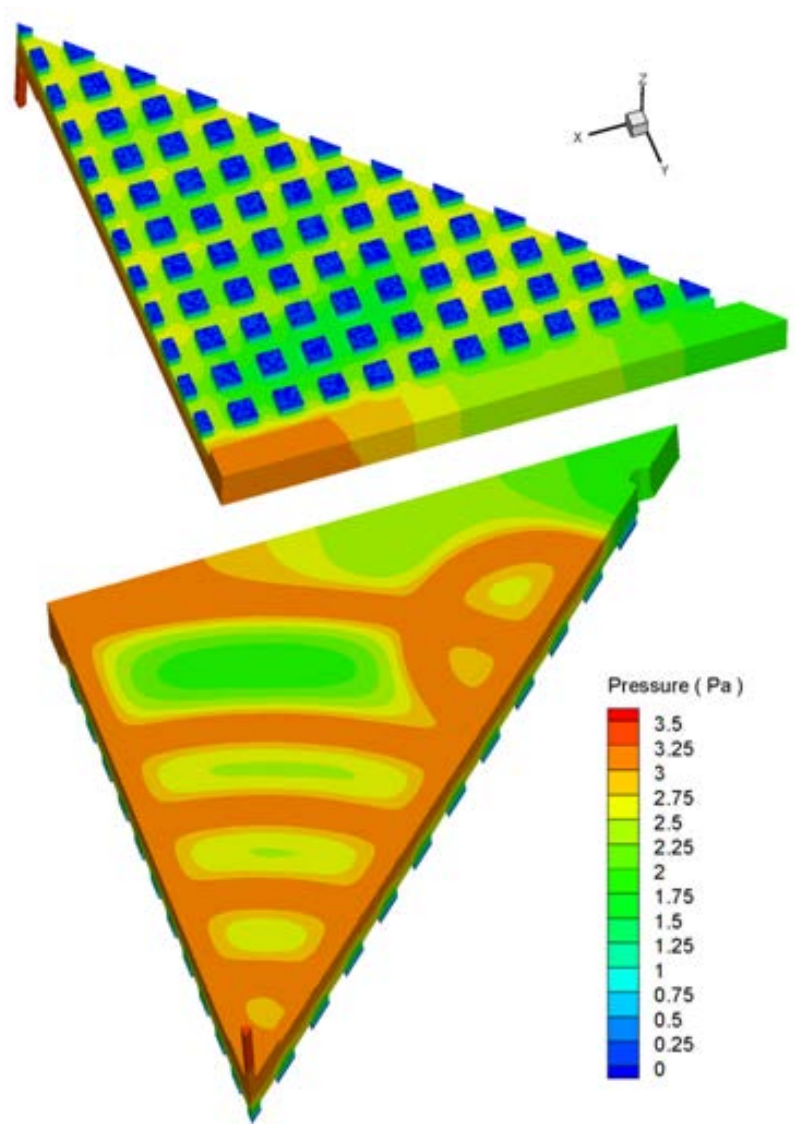

Figure 6. Calculated IFDL pressure field

In the lower part of figure 6 one can see the pressure field on the liquid (feeding) side of the IFDL. The brownish areas trace (as expected) the spider-web conduits and have the highest pressure. The pressure diminishes as the liquid hits the land area of the IFDL and starts soaking it. The upper part of figure 6 shows the pressure field on the other side of the IFDL, which is in contact with the anode side of the MEA. The blue areas indicate regions of zero pressure. We assumed that as the liquid emerges from the porous plate it is at atmospheric pressure and is consumed at the anode.

In figure 7 we show the velocity components (up) and the calculated mass flowrate emerging from the IFDL (down). 

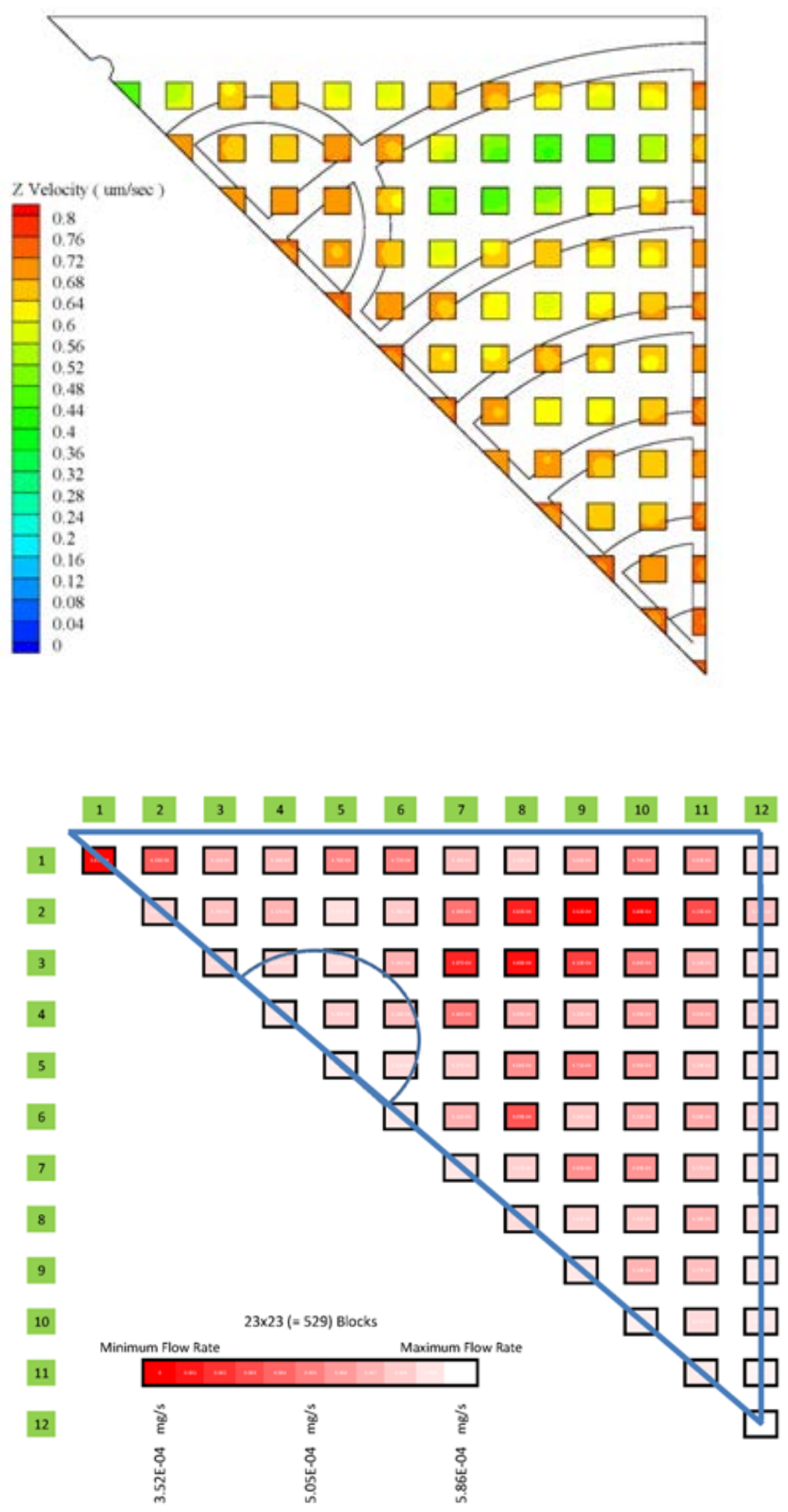

Figure 7. Velocity (up) and mass flowrate (down) at blocks outlet

It can be noticed in the upper figure that the velocity decreases (green vs. yellow and brownish) as we move away from the inlet port (which is the lower tip of the triangle) and as we move 
away from the channel carrying the liquid on the other side of the IFDL. For better illustration the channels on the opposite side of the IFDL are shown in the upper part of figure 7. This has an impact on the fuel discharge and that can be seen in the lower part of figure 7 . Where the color is deep red the discharge rate is lower and therefore the fuel distribution is not uniform. In the areas where the fuel discharge is lower the cell could experience high over-potential and therefore lower energy output.

The problem of non-uniform fuel distribution can also be seen easily in figure 8 which illustrates the flow path lines through the IFDL.

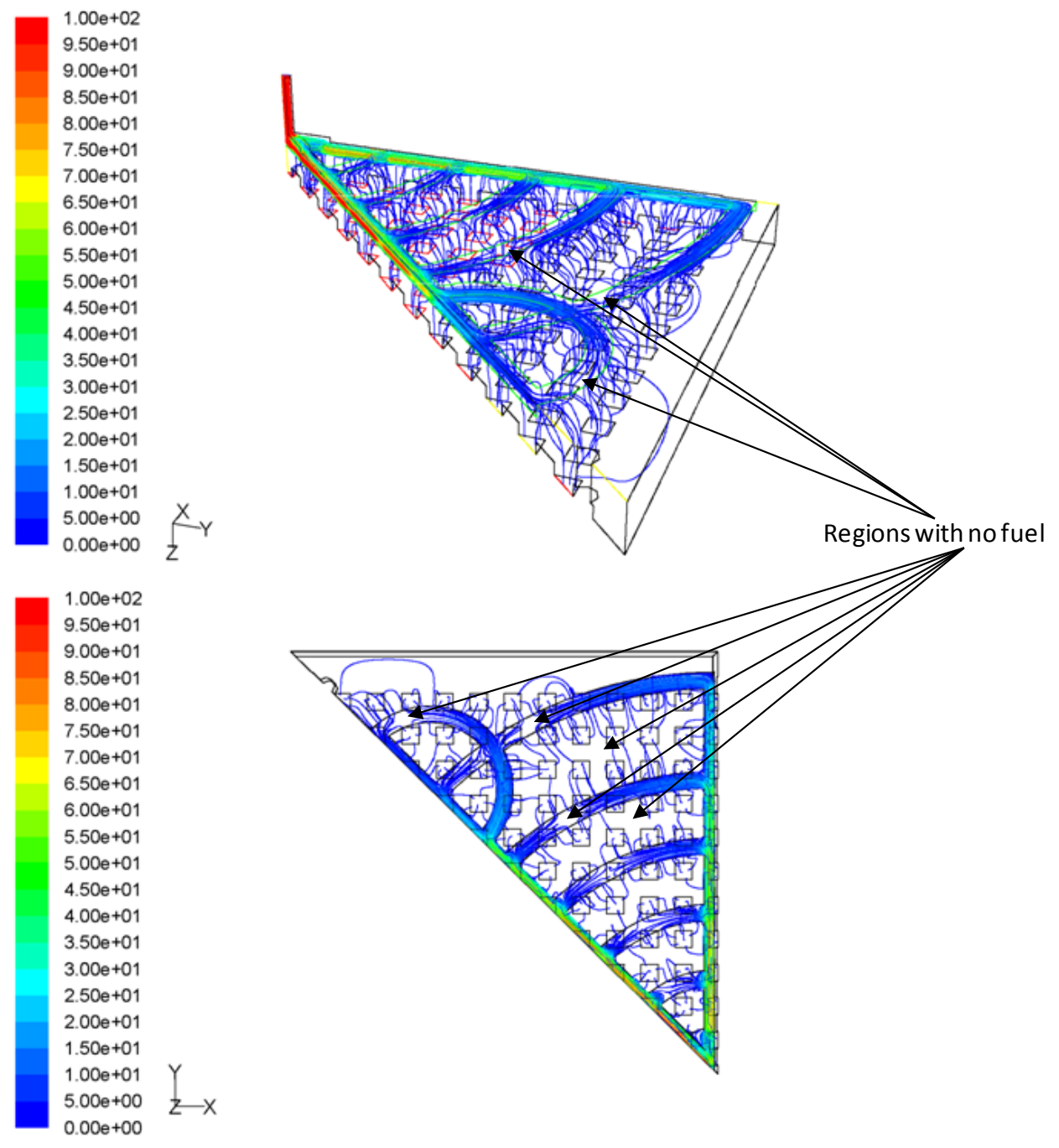

Figure 8. Pathlines of fuel distribution.

A new geometry for the flow field is required

The best structure that we modeled is shown in figure 9 . 


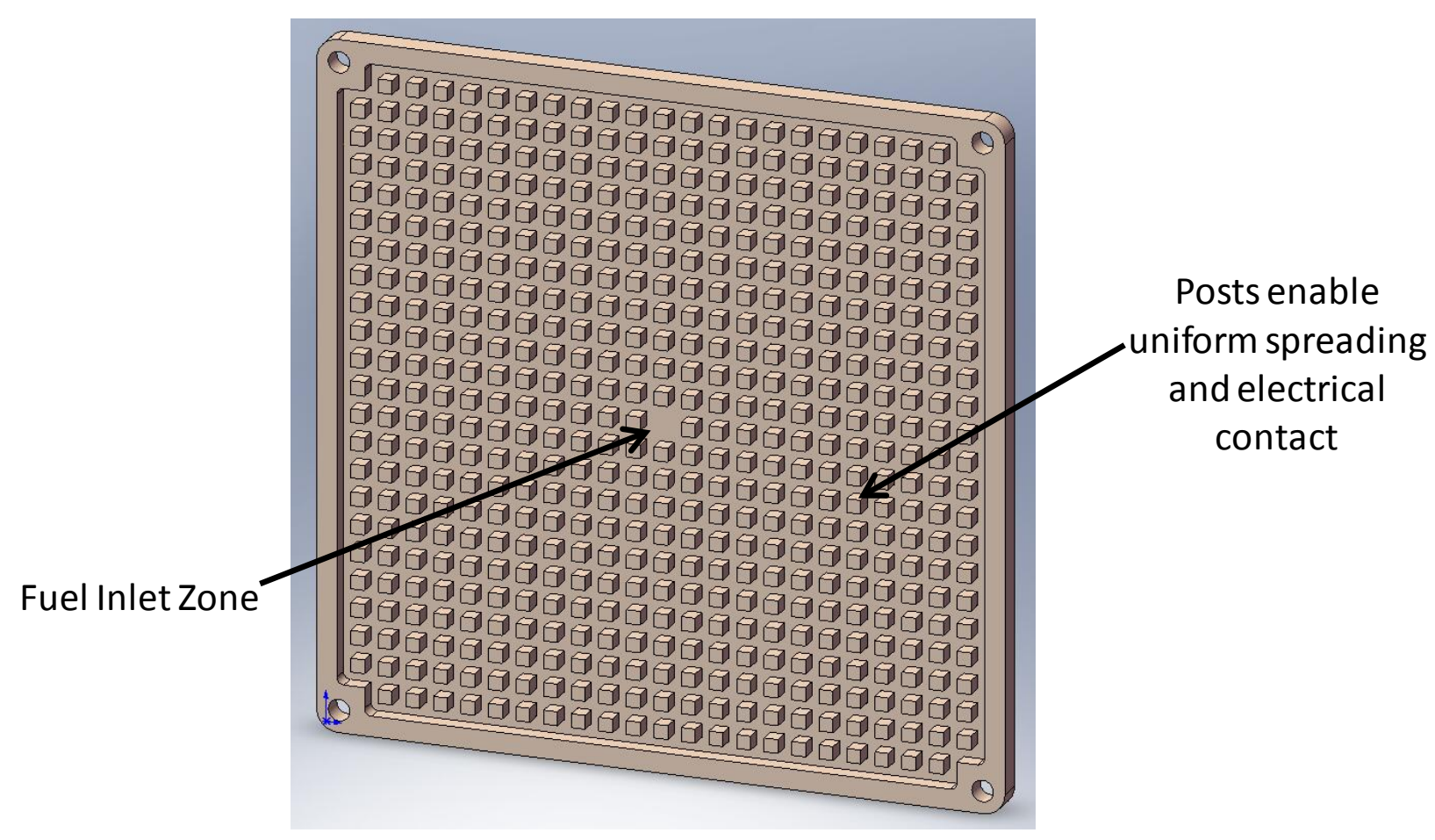

Figure 9. The new and improved fuel side of the IFDL

Fuel enters in the middle of the plate just like in the previous design and then spreads laterally and fills up the entire flow field. The posts ensure a better fuel distribution and also provide intimate electrical contact. The gas evacuation flow field situated opposite the fuel side shown in figure 3 is largely unchanged from the previous design and is shown in figure 10. 


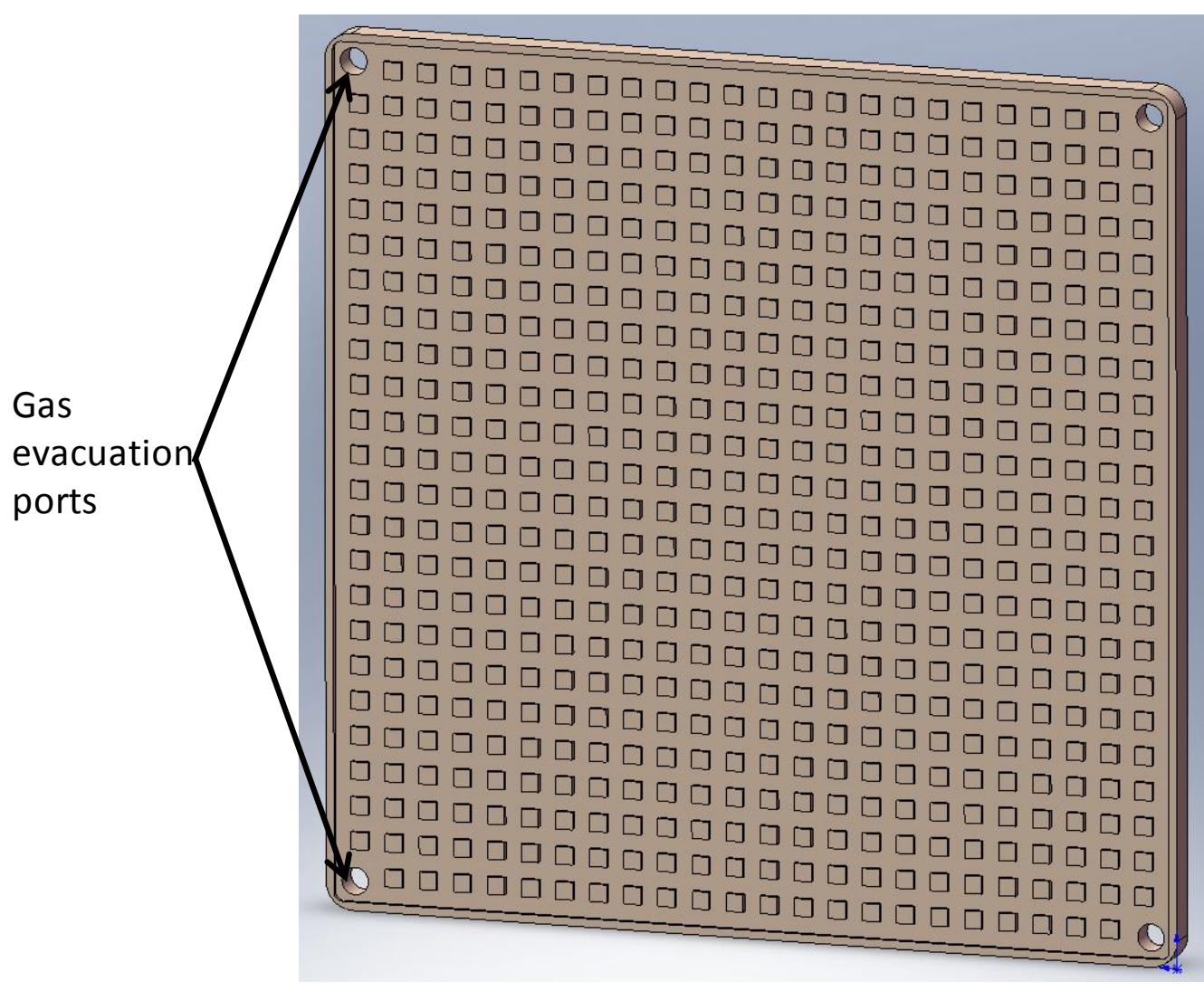

Figure 10. The gas evacuation side of the new IFDL plate

The posts on the gas evacuation side of the IFDL are either in direct contact with the anode catalyst layer or with an additional diffusion layer made of carbon paper or carbon cloth. The channels on the liquid side of the IFDL sit directly above the posts on the gas evacuation side so most of the liquid comes from the liquid side to the gas side through the posts on the gas side. The structure is staggered, i.e. where there is a channel on the liquid side there is a post on the gas evacuation side. This ensures proper distribution of the liquid to the anode catalysts.

\section{Boundary Conditions}

The same boundary conditions as in our previous modeling effort are used:

1) Inlet flowrate: $0.016 \mathrm{gm} / \mathrm{min}$ of $\mathrm{MeOH}$ solution at whatever concentration

2) Inlet pressure: 1 psi above atmospheric pressure (1 psig)

3) Inlet temperature: $60^{\circ} \mathrm{C}$

4) Average pore diameter: 10 microns

5) Total porosity: $40 \% \& 80 \%$ 
In addition to these, 2 geometries were investigated:

1) The channels on both sides have equal size and depth

2) The channels on the liquid side are deep and the channels on the gas side are shallow

The meshing of the new structure having the equal depth geometry is shown in figure 11:

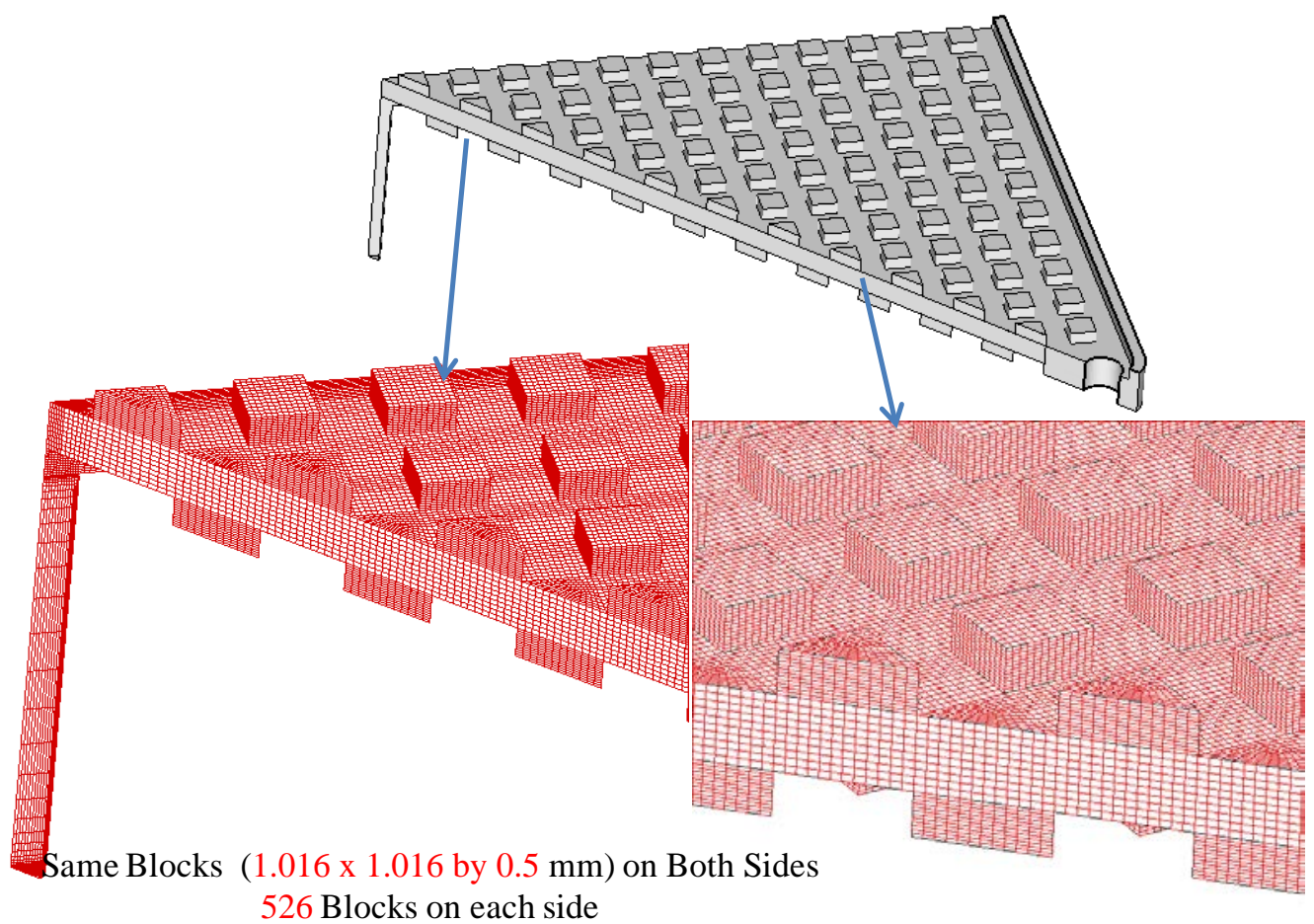

Figure 11: The meshing of IFDL plate with equal depth channels

We have also investigated how 2 different porosities impact the uniformity of flow distribution. The velocity fields are shown in figure 12 : 


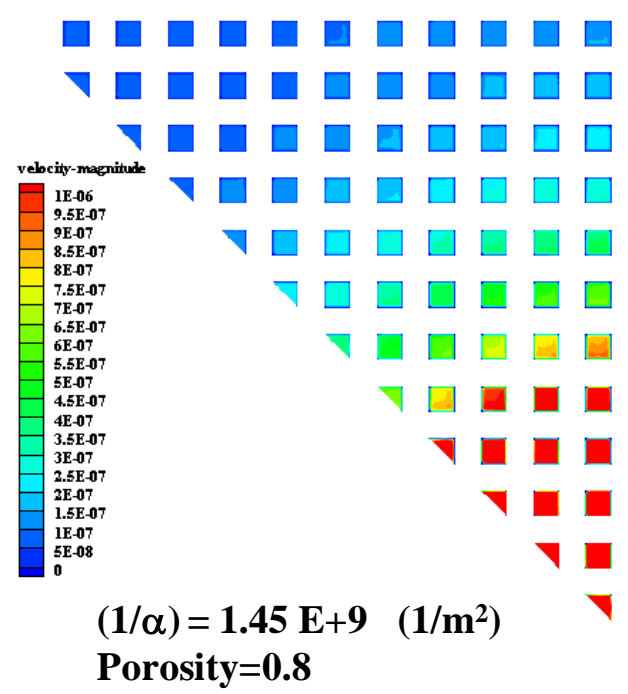

\section{Methanol Velocity on the Lands}

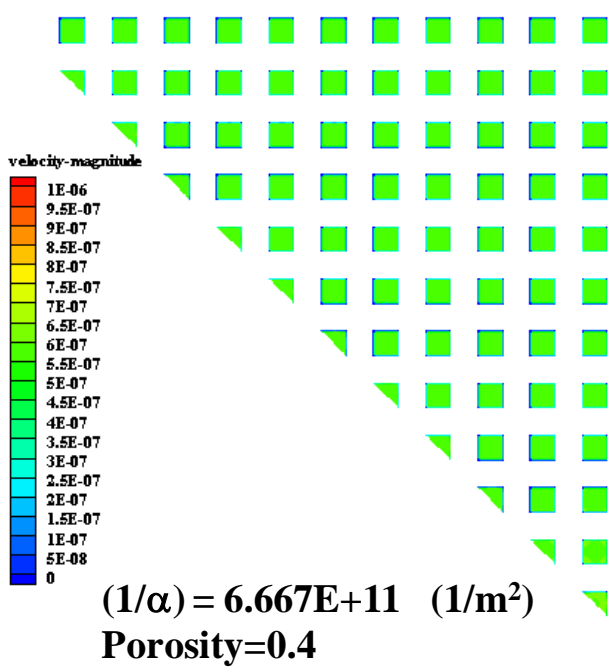

Figure 12: Velocity fields for the 2 various porosities on the equal depth IFDL

It is quite obvious that a fairly large degree of flow non uniformity will develop if a plate with large porosity is employed. Basically, most of the liquid will go through the porous structure on a fairly small radius around the entrance port (the red in the upper left hand side figure) while nothing will remain available at the extremity of the plate (the blue in the upper left hand side figure). By contrast, very uniform distribution is achieved if lower porosity is employed (green in the lower right hand side figure).

The same is easily noticeable in the flow path vector field which is shown in figure 13. 


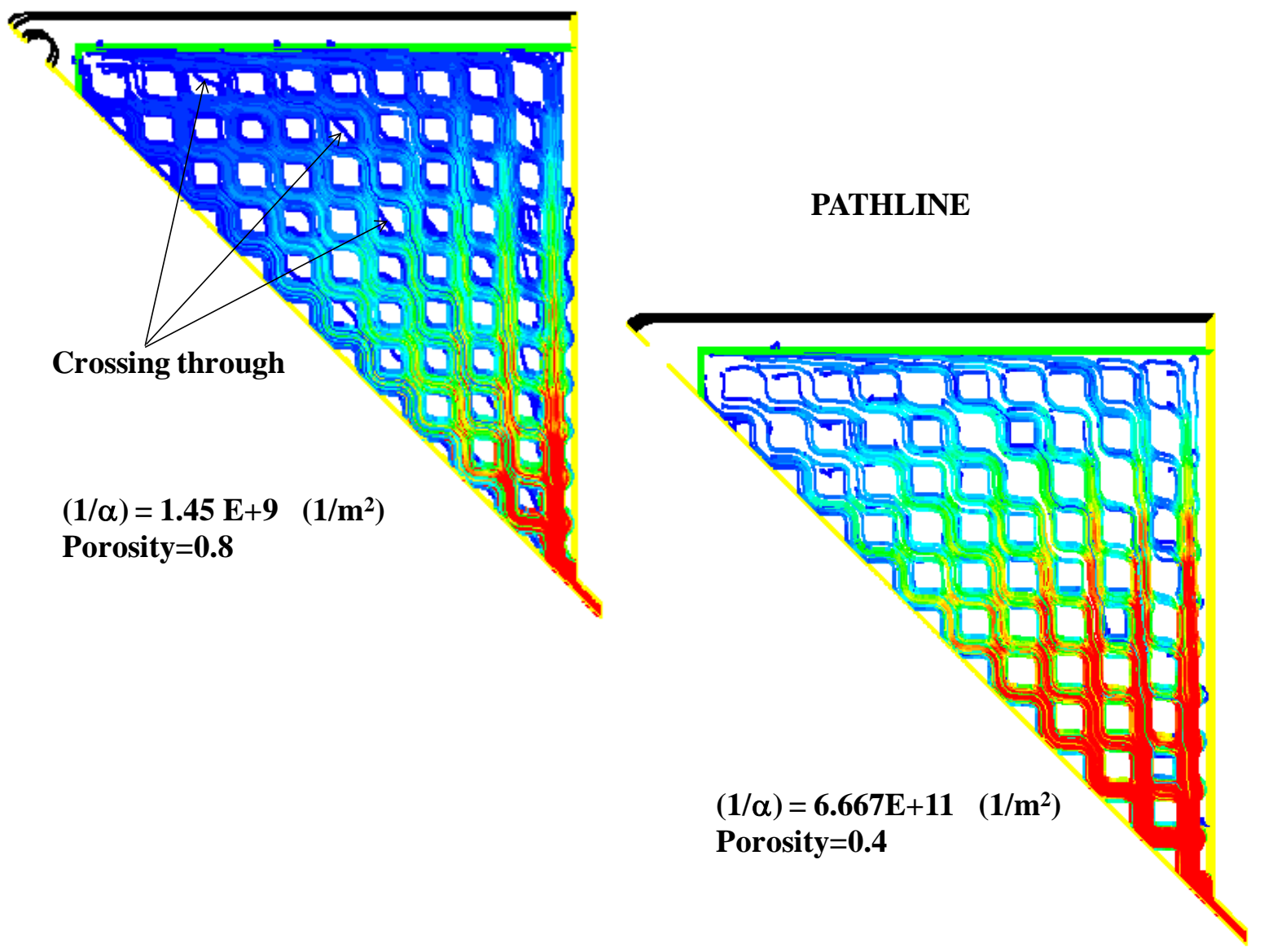

Figure 13. Pathlines for the 2 different porosities

One can very easily notice that in the case of large porosity a significant portion of the modeled wedge remains un/under-utilized (the blue in the figure 13).

Moving on to the structure that has deep channels on the liquid side and shallow channels on the gas side, the meshing is shown in figure 14. 


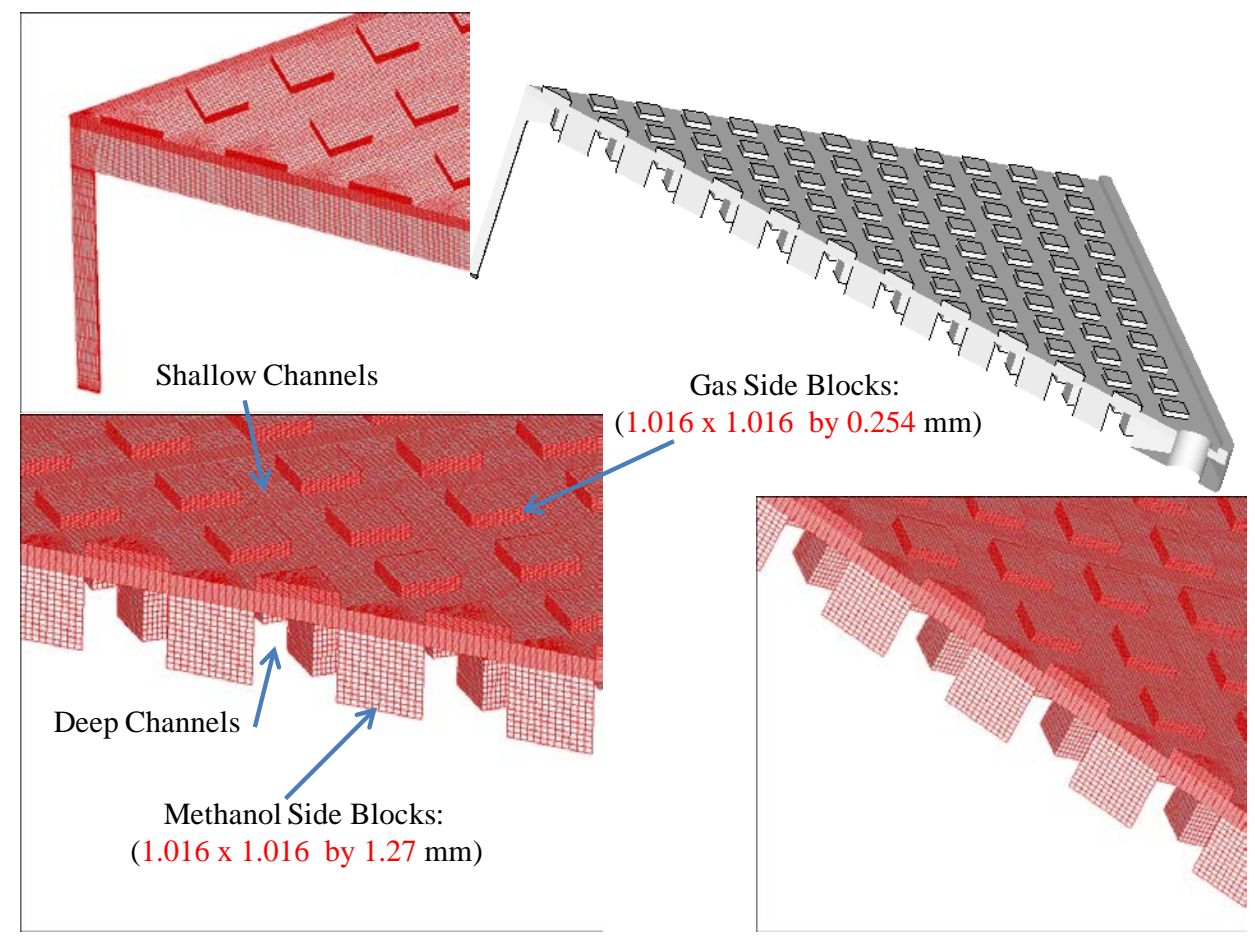

Figure 14. Meshing of the IFDL structure with un-equal depth channels

The velocity fields in this situation are presented in figure 15:

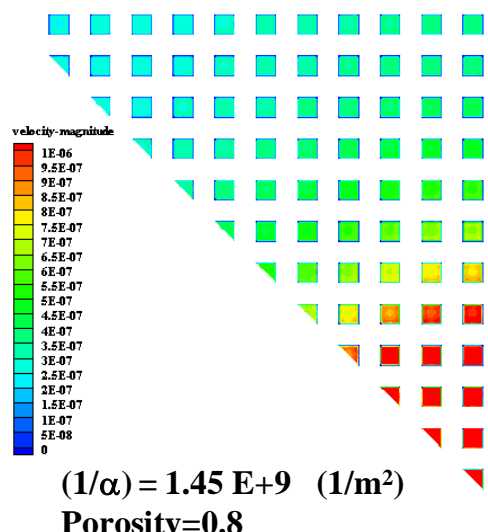

Methanol Velocity on the Lands

Porosity $=0.8$

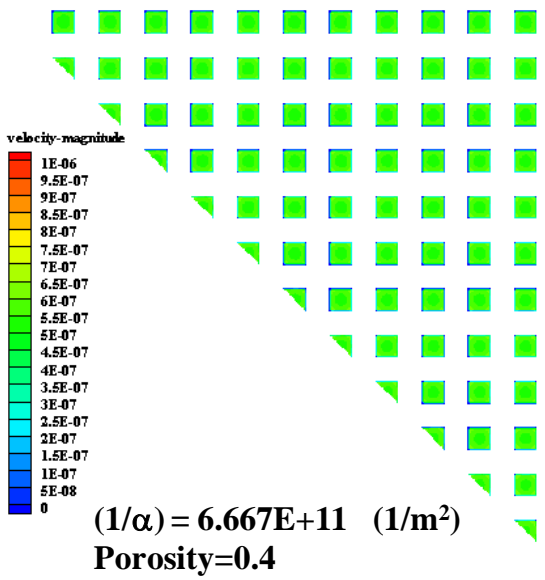

Figure 15. Velocity fields for the 2 various porosities on the un-equal depth IFDL 
One can easily notice that in this situation even the higher porosity presents a better scenario than in the previous case. Since more methanol will enter the, now, deeper liquid side of the flow field it will have time to reach the extremities of the flow field before it diffuses through albeit in a non-uniform fashion. The lower porosity again proves to be the better solution as it presents again very uniform distribution.

The best way to present the modeling data from these 4 different structures is in terms of percentage flow around the average flow.

The data is shown in figure 16.

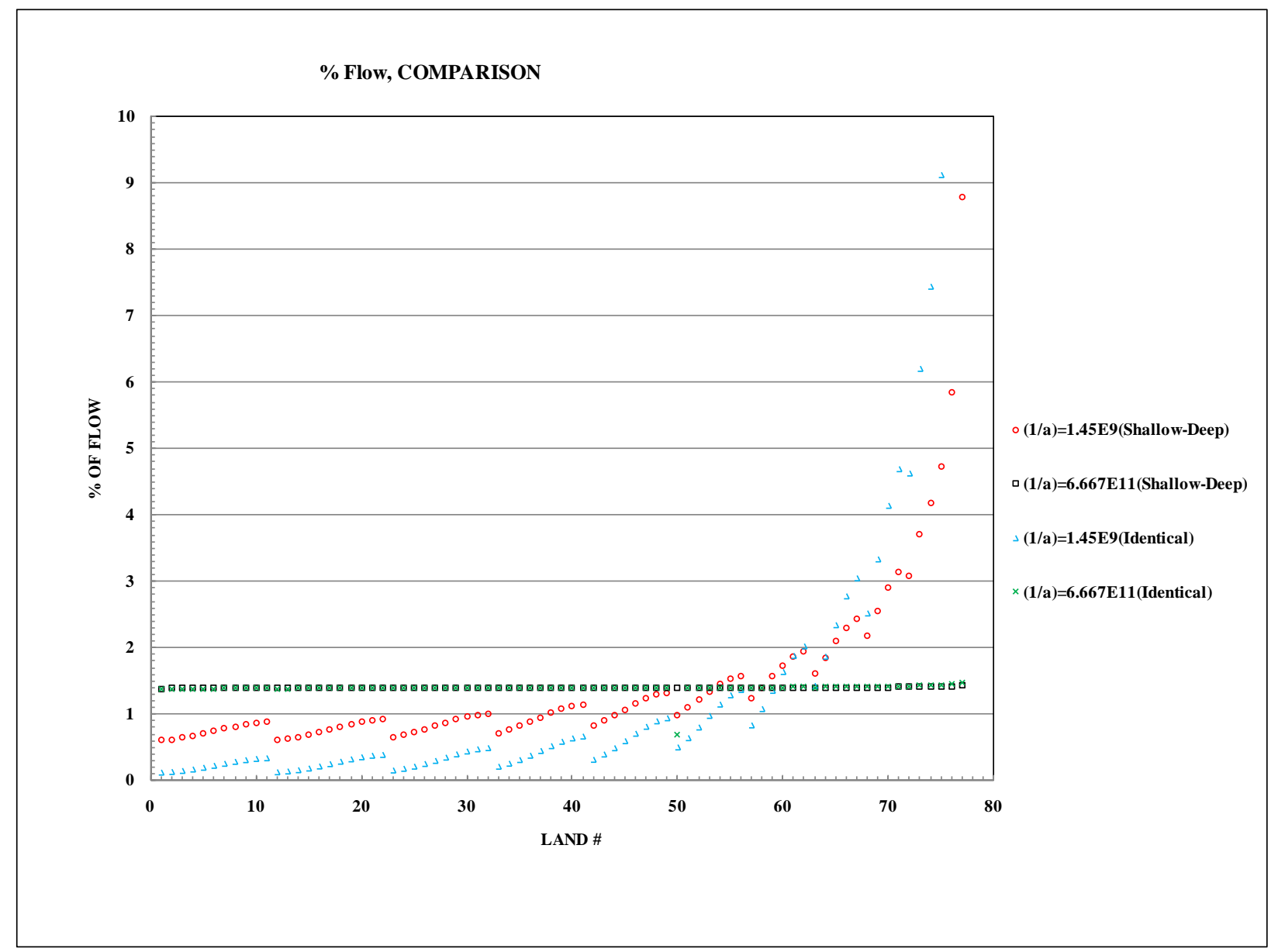

Figure 16. Percentage flow around the average comparison for the 4 structures investigated It can be very clearly seen here that the 2 cases of low porosity (nearly horizontal black square and green "x"s) show extremely uniform flow distribution about the average whereas the 2 cases with high porosity (blue triangles and red circles) show a fairly large deviation from the average. For example, the flowrate of fuel at the entrance (land \# 80) is almost 10\% higher than 
the average which could lead to local overconcentration and fuel and performance loss. Also toward the periphery of the flow field (Land \# 0 ) there is starvation which would lead to tremendous increase in polarization and consequently loss in performance.

The block-block staggered configuration with shallow grooves on the gas evacuation side and low porosity has been retained as the flow field of choice.

\section{Milestone\#2. Fabricate porous plates according to model findings}

Porous plates were produced by compressing graphite powders laced with a binding resin in a mold, followed by a thermal treatment in inert gas that removed the resin partially or in its entirety, leaving behind a porous structure. The porosity and pore diameter were tweaked by varying the pressure at which the powder was compressed as well as the heat treatment parameters. We have commenced the evaluation of the porous plates by acquiring a sample of a porous plate from Porvair to use it as a starting point.

Tables 1-3 show various properties of interest of the Porvair plate.

Table 1. Physical properties of the Porvair plate

\begin{tabular}{|l|l|l|l|l|l|}
\hline Plate & $\begin{array}{l}\text { Average pore } \\
\text { diameter } \\
(\mu \mathrm{m})\end{array}$ & $\begin{array}{l}\text { Median pore } \\
\text { diameter } \\
(\mu \mathrm{m})\end{array}$ & $\begin{array}{l}\text { Bulk } \\
\text { density } \\
(\mathrm{g} / \mathrm{mL})\end{array}$ & $\begin{array}{l}\text { Skeletal } \\
\text { density } \\
(\mathrm{g} / \mathrm{mL})\end{array}$ & $\begin{array}{l}\text { Porosity } \\
(\%)\end{array}$ \\
\hline Porvair & 0.0424 & 2.76 & 1.26 & 1.84 & 32.5 \\
\hline
\end{tabular}

Table 2. Electrical properties of the Porvair plate

\begin{tabular}{|l|l|l|l|}
\hline Plate & $\begin{array}{l}\text { Surface contact resistance } \\
(\text { Gold reference })(\mathrm{m} \Omega)\end{array}$ & $\begin{array}{l}\text { Surface contact } \\
\text { resistance } \\
(\mathrm{m} \Omega)\end{array}$ & $\begin{array}{l}\text { Bulk resistivity } \\
(\Omega \cdot \mathrm{cm})\end{array}$ \\
\hline Porvair & 110 & 243 & Not measured * \\
\hline
\end{tabular}

* Sample too small to measure

Table 3. Permeation properties of the Porvair plate

\begin{tabular}{|c|c|c|c|}
\hline \multirow[b]{2}{*}{ Plate } & \multicolumn{3}{|c|}{$\begin{array}{c}\text { Permeability }{ }^{*} \\
(\mathrm{~kg} / \mathrm{m} \cdot \mathrm{s} \cdot \mathrm{Pa})\end{array}$} \\
\hline & water & $\begin{array}{l}6 \mathrm{M} \\
\text { methanol/water }\end{array}$ & methanol \\
\hline Porvair disk 2 & $2.88 \times 10-13$ & $4.33 \times 10-10$ & $1.36 \times 10-9$ \\
\hline
\end{tabular}

* Testing conditions: 
Plate thickness: $1.93 \mathrm{~mm}$, Permeable area: $5.07 \mathrm{~cm} 2, \Delta \mathrm{P}=20 \mathrm{psig}$

Permeance $=$ mass permeated/permeable area/time/pressure drop, $\mathrm{kg} / \mathrm{m} 2 \cdot \mathrm{s} \cdot \mathrm{Pa}$

Permeability $=$ permeance $\times$ plate thickness, $\mathrm{kg} / \mathrm{m} \cdot \mathrm{s} \cdot \mathrm{Pa}$.

Figure 17 shows water permeability and Figure 18 shows methanol permeability as a function of pressure drop for the Porvair plate.

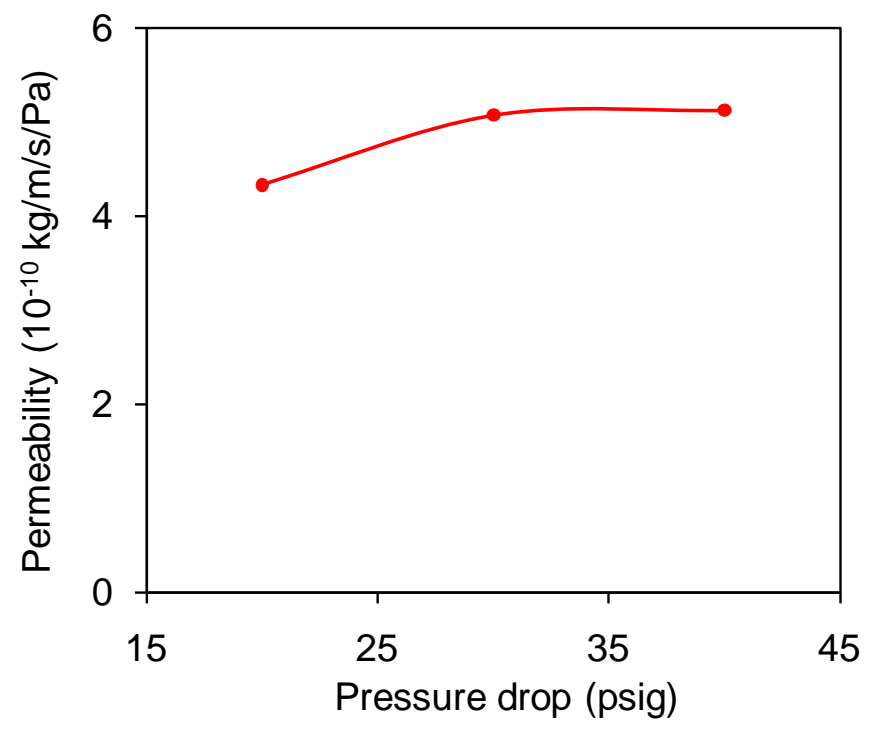

Figure 17. Water permeability

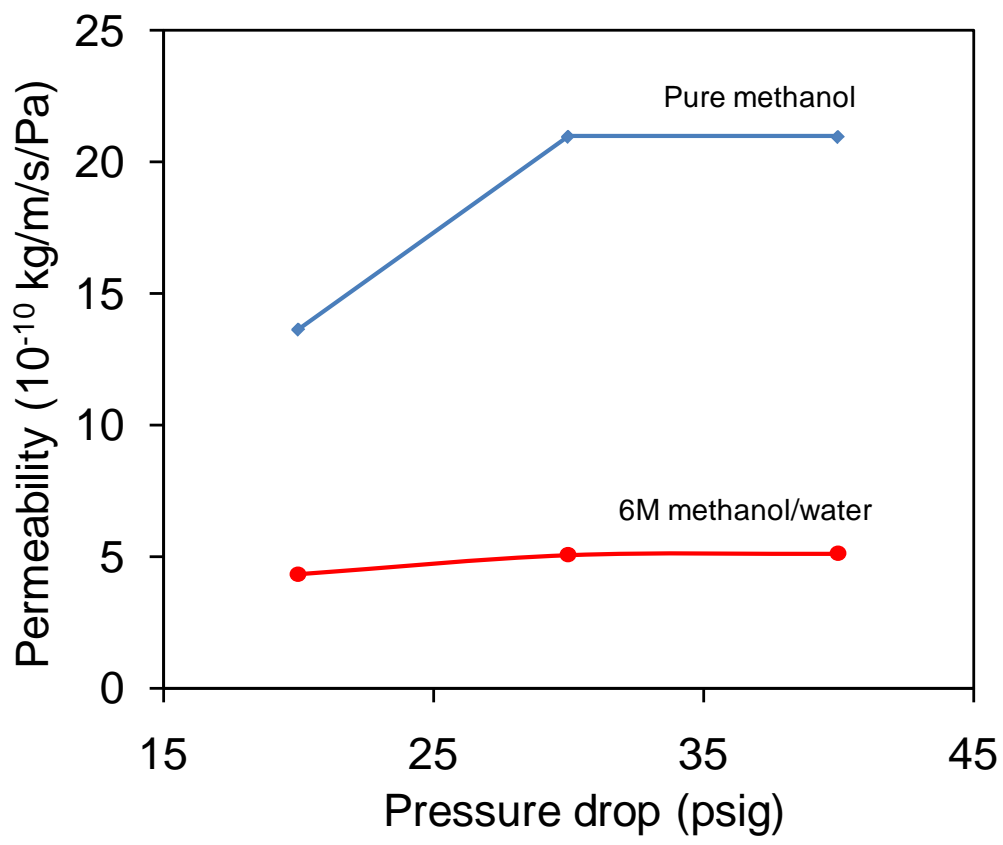

Figure 18. Methanol permeability 
Various porous plates were produced according to the powder compression recipe described previously in the form of slabs, coupons were cut off for analysis and flowfields were engraved in them using a CNC machine. The cycle of fabrication-characterization-flow field machining is shown in figure 19.

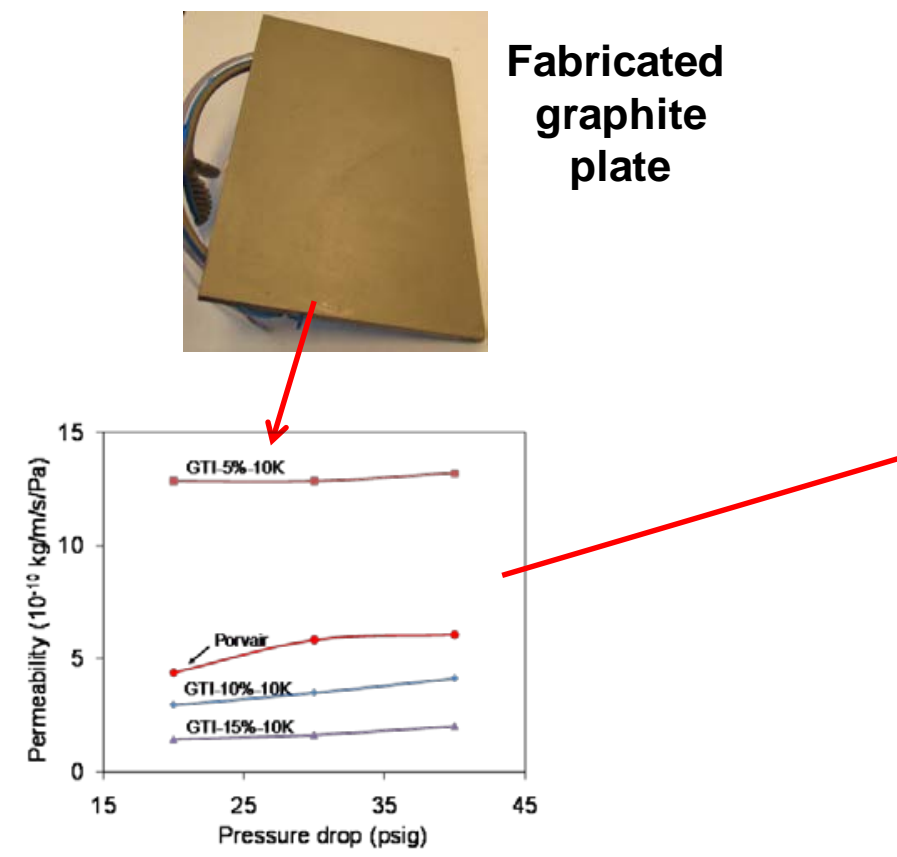

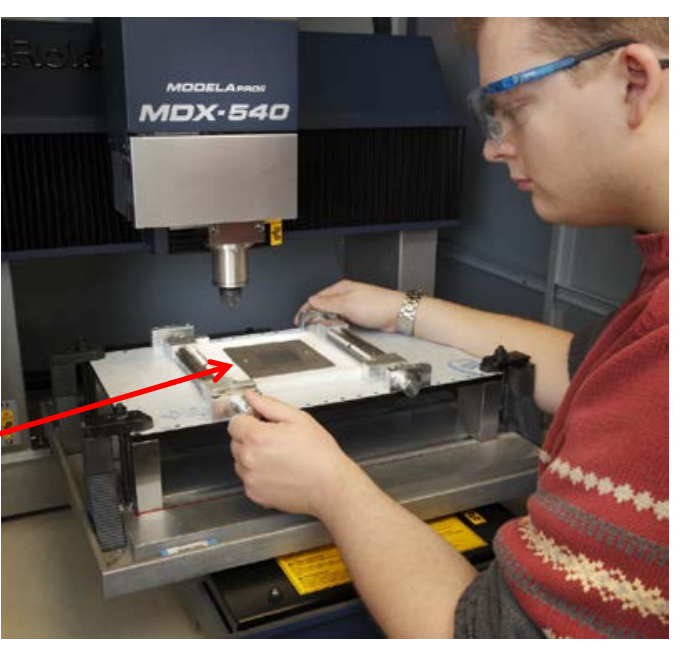

Machining using CNC

Figure 19. Cycle of fabrication of the porous plate (left up), characterization (left down) and machining the flowfield (right)

Physical properties of the plates were determined by GTI Analytic Lab. Results are summarized in Table 4. The physical properties for Porvair plate are also listed in Table 4 for comparison.

Table 4. Comparison of physical properties

\begin{tabular}{|c|c|c|c|c|c|}
\hline Plate & $\begin{array}{c}\text { Average pore } \\
\text { diameter } \\
(\mu \mathrm{m})\end{array}$ & $\begin{array}{c}\text { Median pore } \\
\text { diameter } \\
(\mu \mathrm{m})\end{array}$ & $\begin{array}{c}\text { Bulk } \\
\text { density } \\
(\mathrm{g} / \mathrm{mL})\end{array}$ & $\begin{array}{c}\text { Skeletal } \\
\text { density } \\
(\mathrm{g} / \mathrm{mL})\end{array}$ & $\begin{array}{c}\text { Porosity } \\
(\%)\end{array}$ \\
\hline Porvair & 0.0424 & 2.76 & 1.26 & 1.84 & 32.5 \\
\hline GTI-5\%-10K* & 0.646 & 2.73 & 1.36 & 2.11 & 36.3 \\
\hline GTI-10\%-10K & 0.505 & 2.50 & 1.57 & 2.10 & 26.2 \\
\hline GTI-15\%-10K & 0.279 & 2.82 & 1.55 & 2.03 & 24.6 \\
\hline
\end{tabular}

* GTI-5\%-10K means the plate was prepared with 5\% resin (binder) and pressed at 10K lbsforce 
We have produced porous plates of various properties with respect to pore diameter and porosity. These two parameters ultimately impact the permeability of the plate. More examples of plates are shown below.

Electrical properties of the plates are summarized in Table 5. Results for the Porvair plate are also listed in Table 5 for comparison.

Table 5. Comparison of electrical properties

\begin{tabular}{|l|l|l|}
\hline Plate & $\begin{array}{l}\text { Surface contact resistance* } \\
(\mathrm{m} \Omega)\end{array}$ & $\begin{array}{l}\text { Volume resistivity } \\
(\Omega \cdot \mathrm{cm})\end{array}$ \\
\hline Porvair & 243.3 & $-^{\star \star}$ \\
\hline GTI-5\%-10K & 213.7 & $3.96 \times 10-4$ \\
\hline GTI-10\%-10K & 207.9 & $2.86 \times 10-4$ \\
\hline GTI-15\%-10K & 195.6 & $2.22 \times 10-4$ \\
\hline GTI-5\%-33K & 206.7 & $1.98 \times 10-4$ \\
\hline GTI-5\%-66K & 198.7 & $1.13 \times 10-4$ \\
\hline GTI-5\%-99K & 197.8 & $1.12 \times 10-4$ \\
\hline GTI-5\%-132K & 178.0 & $1.27 \times 10-4$ \\
\hline GTI-5\%-165K & 172.6 & $1.03 \times 10-4$ \\
\hline
\end{tabular}

* During measurement for each sample, the surface contract resistance of gold was measured as reference. The values for gold were $98-110 \mathrm{~m} \Omega$

** Sample too small to measure.

Hydraulic permeabilities of pure water, pure methanol, and methanol/water mixtures for the Porvair and the fabricated plates were measured using the system shown in Figure 20. Permeabilities were generally measured at pressure drops of 20, 30 and 40 psig. Note that permeability $(\mathrm{kg} / \mathrm{m} \cdot \mathrm{sec} \cdot \mathrm{Pa})$ was calculated by permeance $\times$ plate thickness, where permeance $\left(\mathrm{kg} / \mathrm{m}^{2} \cdot \mathrm{sec} \cdot \mathrm{Pa}\right)=$ mass permeated $/$ permeable area/time/pressure drop. 


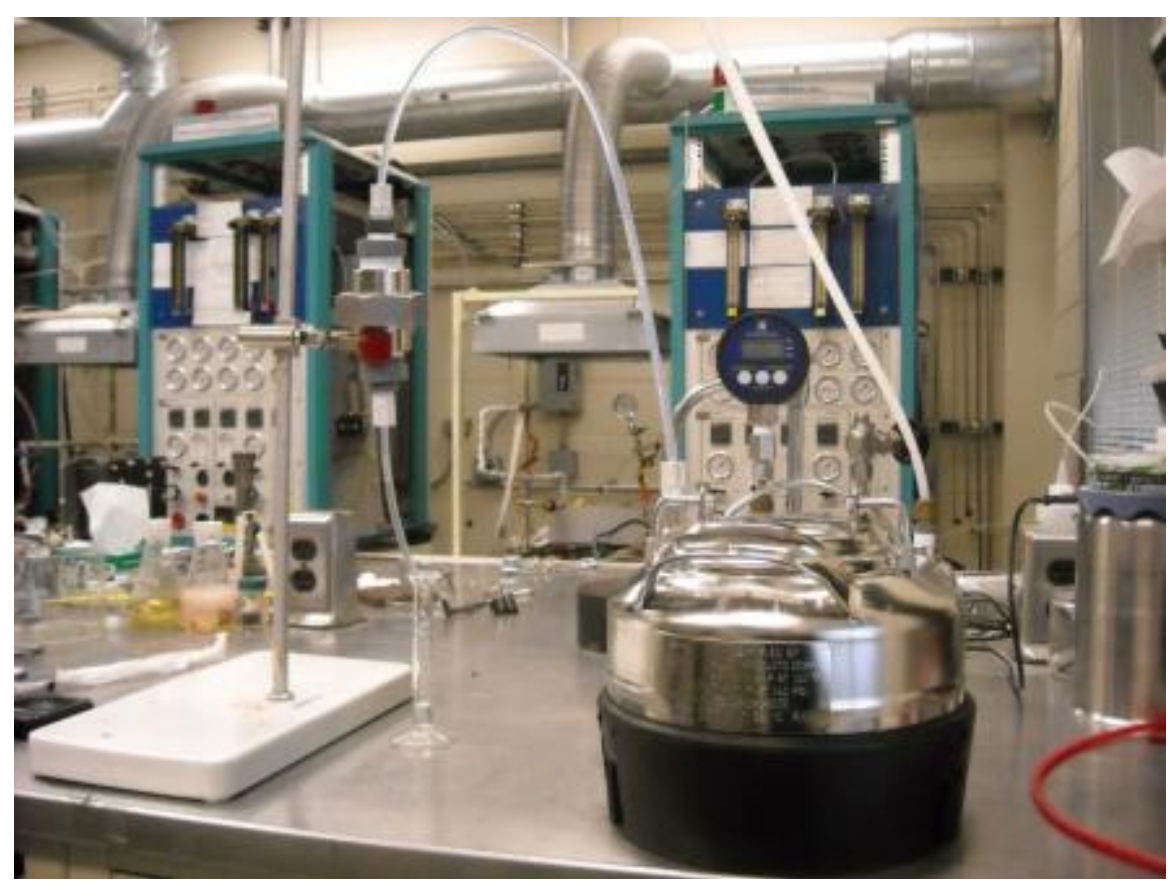

Figure 20. Hydraulic permeability testing system

Figure 21 compares permeabilities for a 50/50 (vol\%) methanol/water (12.5 M) of fabricated plates with Porvair plate. In the pressure drop range measured, the permeabilities of Porvair plate were in between those plates prepared with different fractions of binder. To insure that methanol does not interact with the resin and/or the graphite methanol permeate concentrations were measured by off-line gas chromatograph (CARLE Series 400) equipped with a thermal conductivity detector and HAYESEP-A column. For all plates, the measured methanol concentrations in the permeate side were identical to those in the feed.

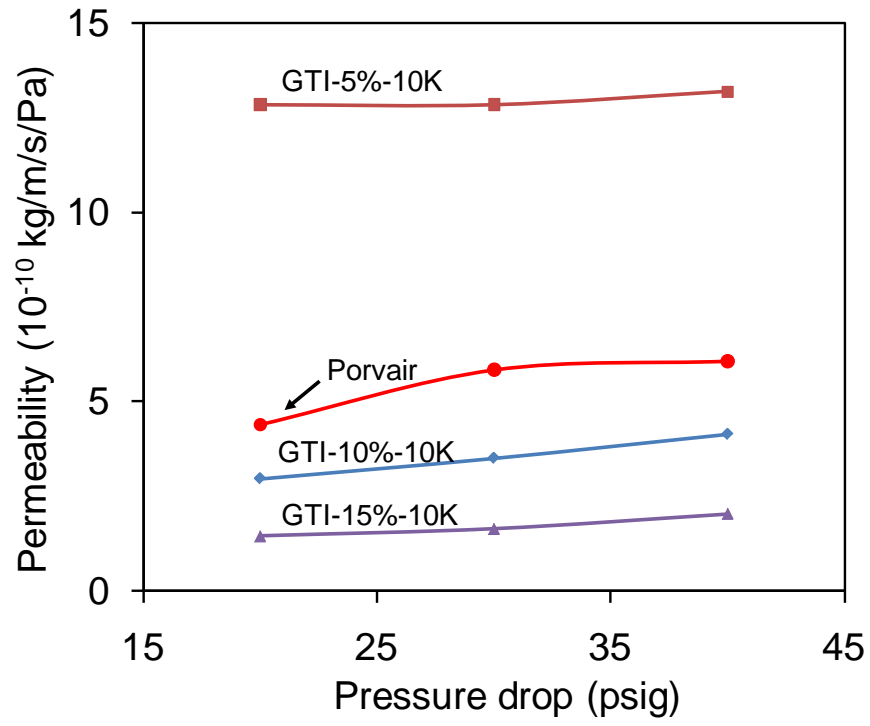

Figure 21. Permeability for 50/50 vol\% methanol/water mixture 
Figure 22 compares permeabilities of Porvair and GTI-5\%-10K plates at a pressure drop of 20 psig for different methanol feed concentrations. Pure water, $6 \mathrm{M}, 12.5 \mathrm{M}, 17.6 \mathrm{M}$ methanol/water mixtures, and pure methanol feeds have been investigated. Apparently, Porvair plate was highly hydrophobic as its water permeability was much lower than the GTI-5\%-10K plates.

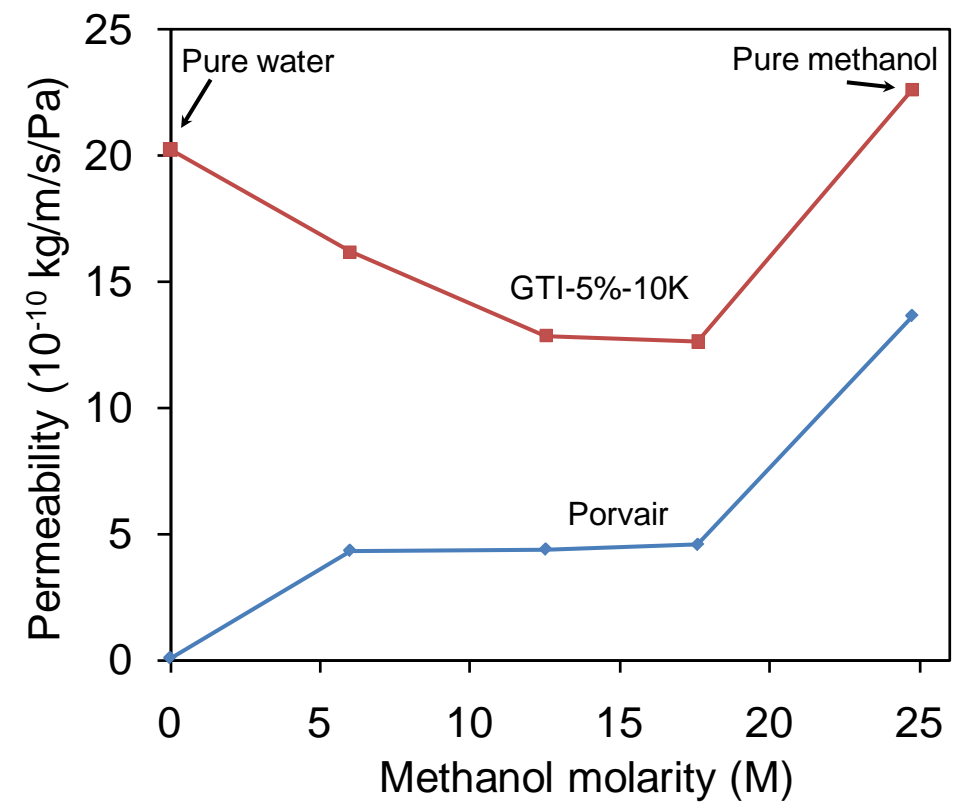

Figure 22. Permeabilities at a pressure drop of 20 psig for Porvair and GTI-5\%-10K plates.

Note that the permeabilities for GTI-5\%-10K plates were much higher than those for Porvair plates with all feeds measured:

- For 6M methanol/water, $\quad$ PGTI-5\%-10K = 3.7 times of Porvair

- For 12.5M methanol/water (50/50 vol\%), PGTI-5\%-10K = 2.9 times of Porvair

- For 17.6M methanol/water (50/50 mol\%), PGTI-5\%-10K = 2.8 times of Porvair

- For pure methanol, $\quad$ PGTI-5\%-10K = 1.7 times of Porvair

Several plates were chosen to be machined and tested in the fuel cell.

Using a Modela PRO II MDX-540 CNC machine flow fields were engraved into the porous plates according to the modeling results obtained previously.

Examples of machined porous plates are shown in figure 23. 

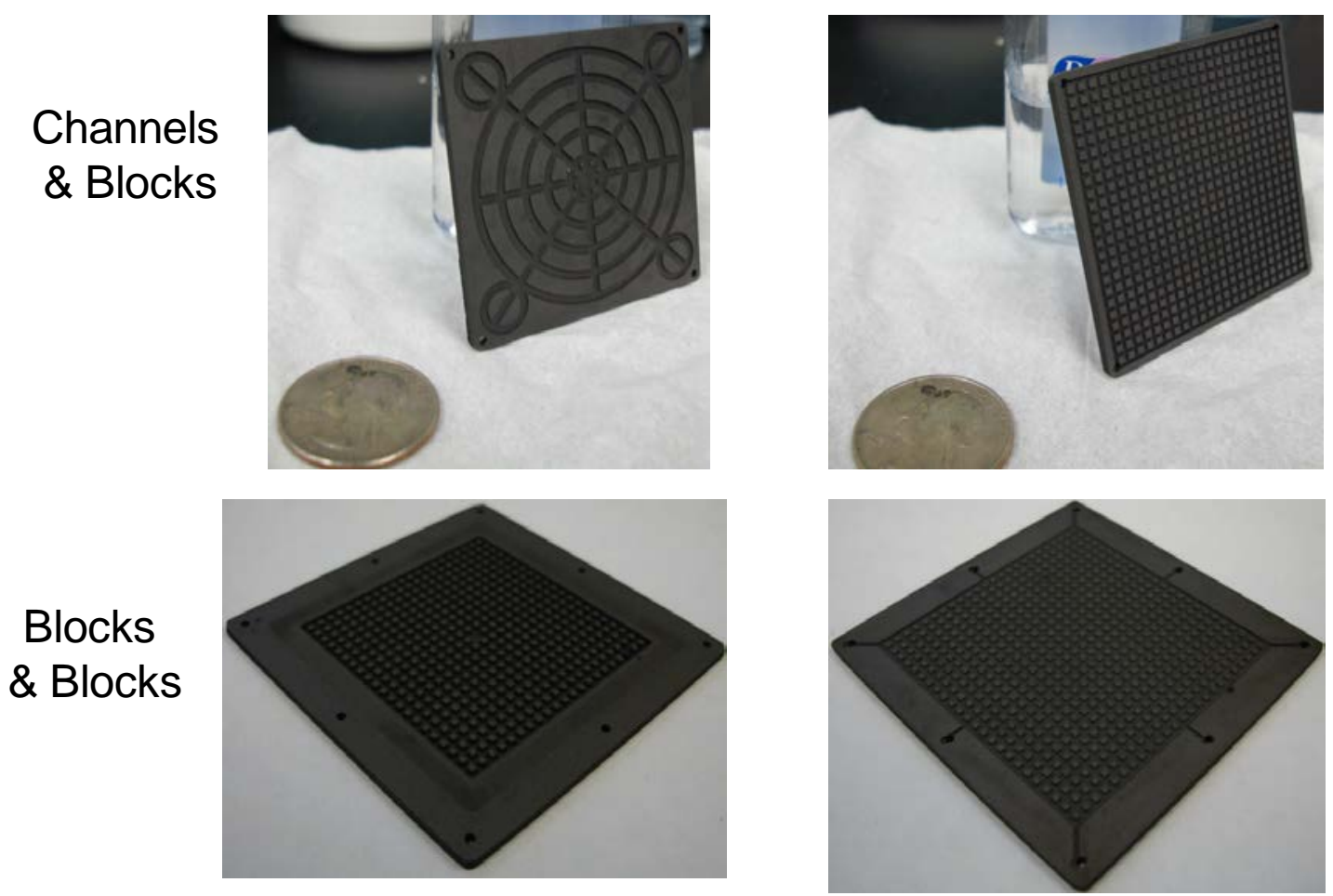

Figure 23. Examples of machined porous plates.

Upper Row: unoptimal spider web design (left: fuel inlet side, right gas evacuation side) Lower Row: optimal block-block design (left: fuel inlet side, right gas evacuation side)

Housing hardware for the porous plates was designed using Solidworks and was manufactured out of brass which was plated with gold to ensure corrosion resistance under the operating conditions of the fuel cell. The housing is shown in figure 24.

\section{Anode Housing}

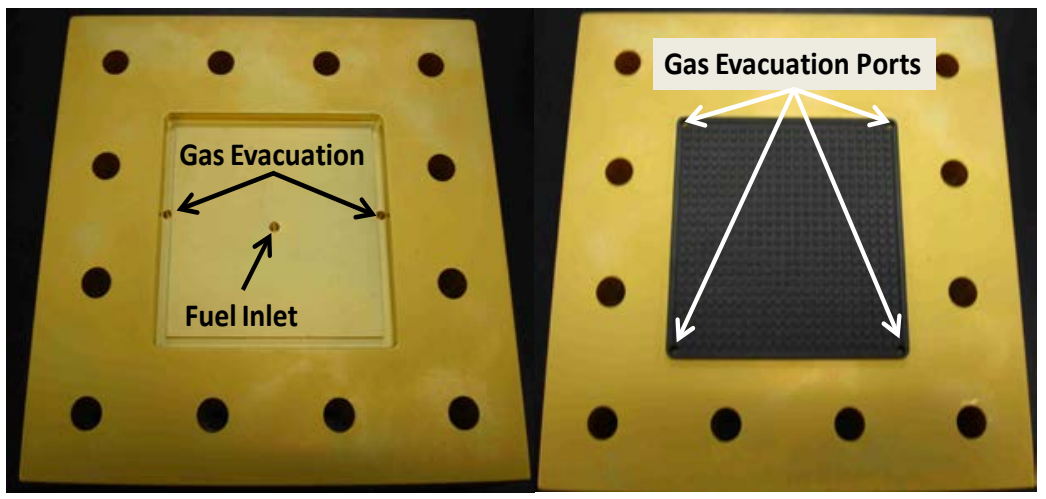

Cathode Housing

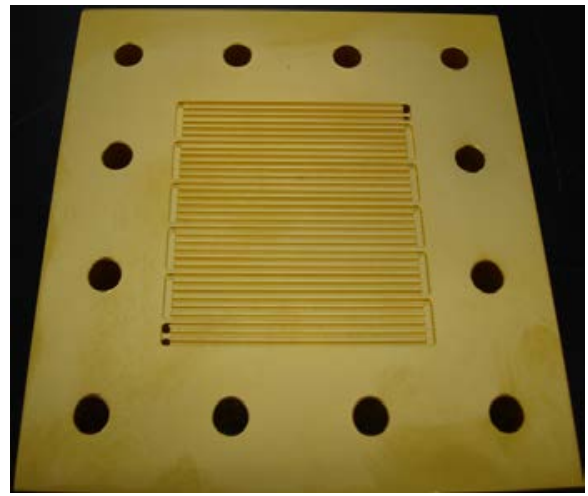

Figure 24. The hardware used for DMFC testing using the fabricated porous plates.

The porous plate snaps into the anode housing (left two pictures) and a conventional serpentine is used for the cathode housing (right) 
It is easy to understand how the fluid admission and gas evacuation are being accomplished by looking at figure 24 . In the left hand side panel one can see the central port where fuel injection occurs. The fluid spreads out and fills up the open volume situated on the liquid admission side of the IFDL. The liquid (concentrated methanol) diffuses through the plate and reacts at the anode side releasing $\mathrm{CO}_{2}$. The evolved $\mathrm{CO}_{2}$ finds its way toward the corners of the IFDL plate as indicated in the middle picture of figure 24 , collects in the square shaped channel (shown in the left picture of figure 24) and it is evacuated through the 2 gas evacuation ports.

With regards to the multitude of combinations of plates and special treatments fabricated by NuVant we attempted to improve the performance of certain plates by covering the gas evacuation channels with a hydrophobic epoxy. The rationale behind this treatment was that the hydrophobic epoxy would block the permeation of methanol through the gas evacuation channels thus freeing them for the transport of exhaust gas outside of the cell. Concomitantly it was hoped that the outgoing gas would not engage the incoming methanol, transporting it outside of the cell before it had the chance to react and produce electrons thus lowering the fuel efficiency.

An example of such produced plate with NuVant designed flowfield is shown in figure 25.

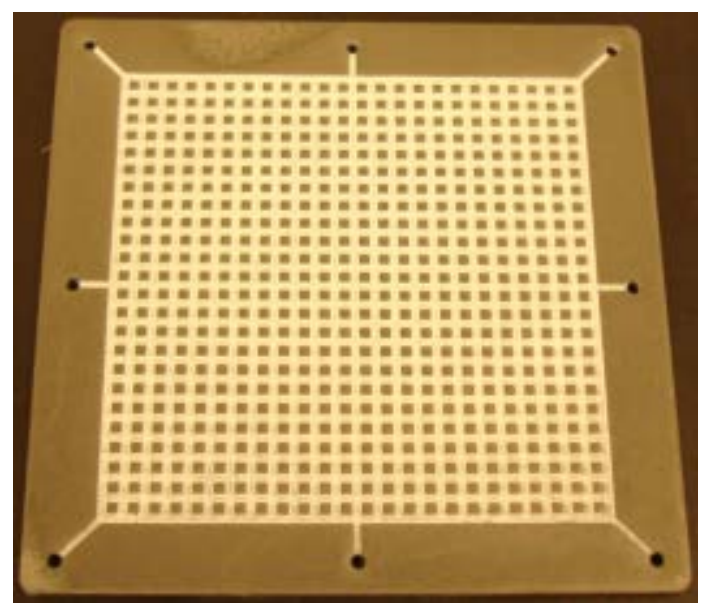

Figure 25. Epoxy-blocked IFDL

For reasons that remain unknown, the epoxy-treated plates showed much poorer performance compared to the untreated plates and NuVant decided to abandon the treatment and focus onto the untreated plates. 


\section{Milestone\#3. Maximize fuel energy density by optimization of MEA} structure and operating scheme \& perform extended life tests of optimized MEAs on single cells

MEAs for the IFDL DMFC were fabricated at NuVant using Johnson Matthey unsupported PtRu as the anode catalyst and Johnson Matthey unsupported Pt as the cathode catalyst. The MEAs were fabricated using the catalyst coated membrane technique where the membrane is immobilized onto a vacuum table and the catalysts are painted onto either side. The loading was always $4 \mathrm{mg} / \mathrm{cm}^{2}$ of catalyst for both the anode and the cathode.

Two modes of fuel cell operation were employed: fuel dosing mode and continuous fuel delivery mode.

\section{Fuel dosing mode}

A fixed dose of methanol of known volume is sent inside the liquid side of the IFDL through the centrally located port. The liquid is allowed to diffuse through the porous plate and is consumed in the anodic reaction at a fixed current. When the fuel is consumed the fuel cell enters a mass transport affected regime and the voltage experiences a sharp drop. This signals that a new dose needs to be delivered. Once the voltage drops to a user-defined value (dose trigger) a new dose is administered and the process repeats. Based on the number of doses and their volume, important fuel cell performance metrics are calculated, like the average power, the fuel utilization, the gross fuel energy density (GFED), etc.

A few examples of results with this type of operation, together with the operating conditions are given in figures 26 a-g.

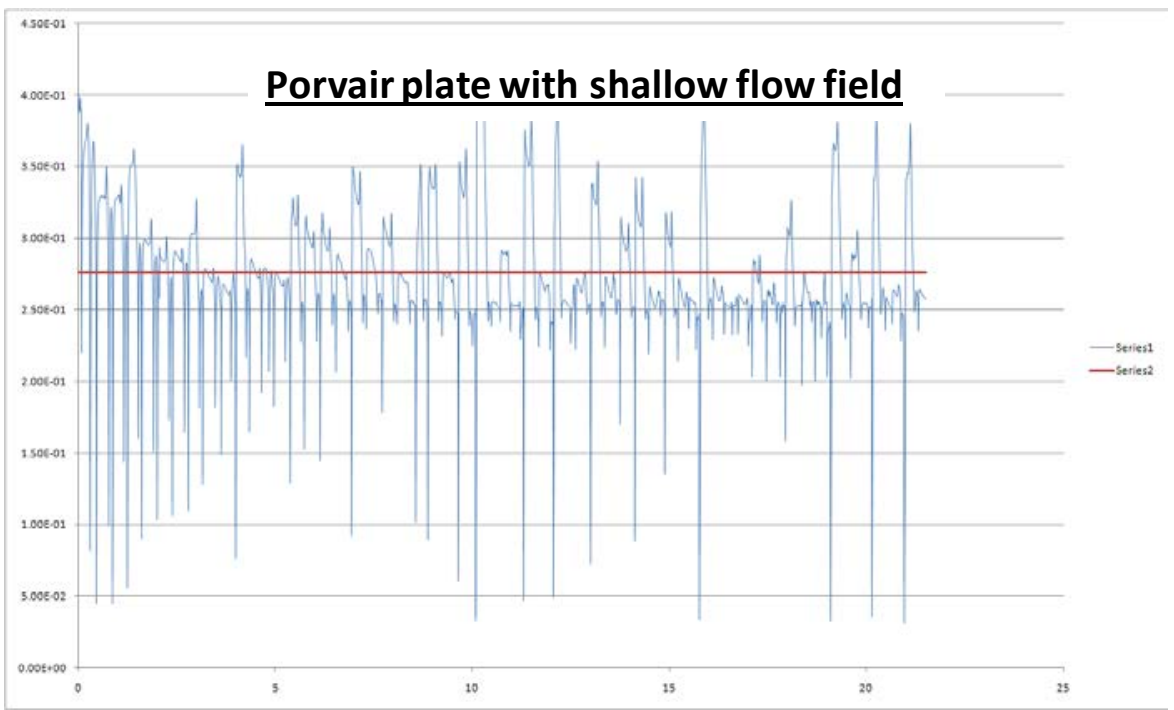

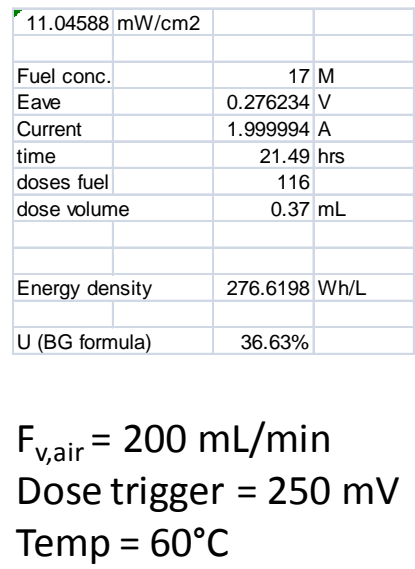


Figure 26a. IFDL DMFC operation in dose mode\#1

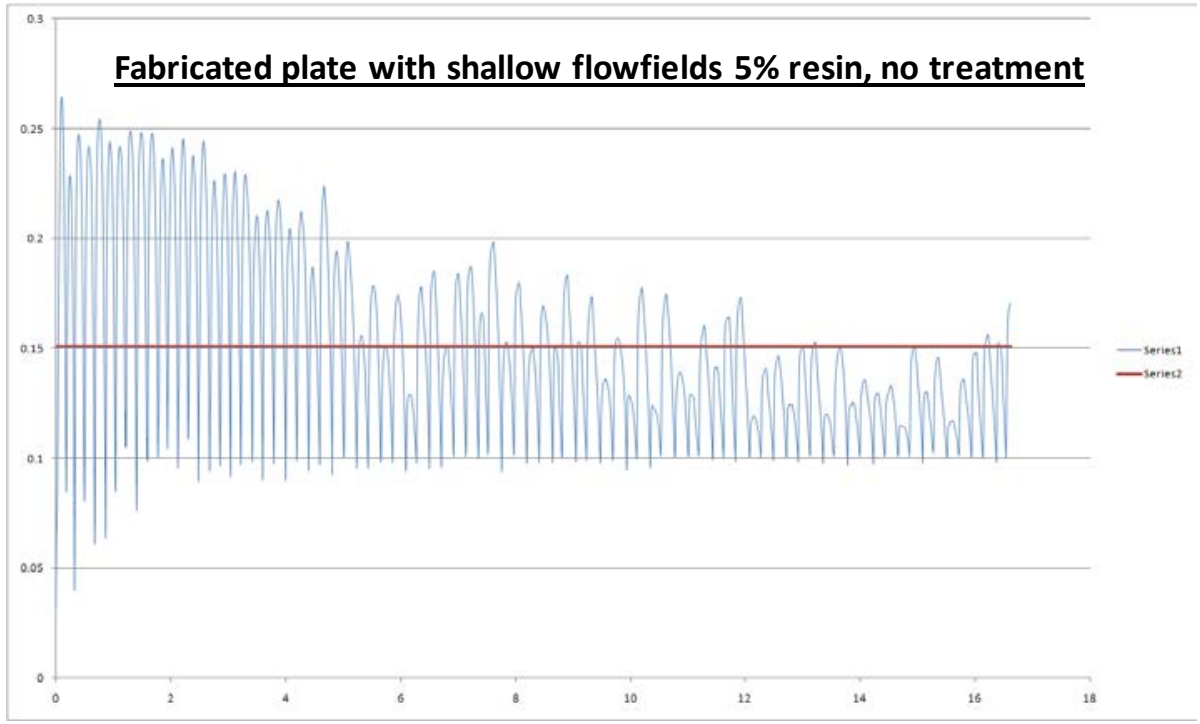

\begin{tabular}{|l|r|}
\hline $12.08573 \mathrm{~mW} / \mathrm{cm} 2$ & \\
\hline Fuel conc. & $17 \mathrm{M}$ \\
\hline Eave & $0.151151 \mathrm{~V}$ \\
\hline Current & $1.999043 \mathrm{~A}$ \\
\hline time & $17.21 \mathrm{hrs}$ \\
\hline doses fuel & 82 \\
\hline dose volume & $0.37 \mathrm{~mL}$ \\
\hline & \\
\hline Energy density & $171.3947 \mathrm{Wh} / \mathrm{L}$ \\
\hline & \\
\hline $\mathrm{U}$ (BG formula) & $41.48 \%$ \\
\hline
\end{tabular}

$\mathrm{F}_{\mathrm{v}, \text { air }}=200 \mathrm{~mL} / \mathrm{min}$

Dose trigger $=100 \mathrm{mV}$

Temp $=60^{\circ} \mathrm{C}$

Figure 26b. IFDL DMFC operation in dose mode\#2

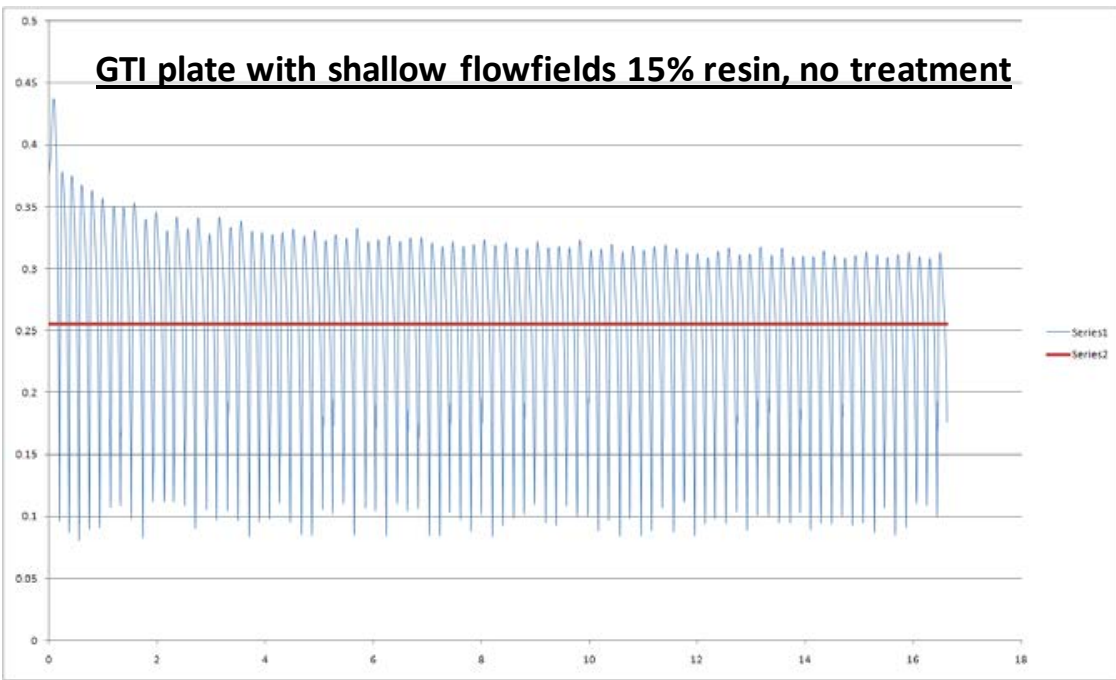

$20.44815 \mathrm{~mW} / \mathrm{cm} 2$

\begin{tabular}{|l|r|}
\hline Fuel conc. & $17 \mathrm{M}$ \\
\hline Eave & $0.255602 \mathrm{~V}$ \\
\hline Current & $2.000003 \mathrm{~A}$ \\
\hline time & $16.62 \mathrm{hrs}$ \\
\hline doses fuel & 84 \\
\hline dose volume & $0.37 \mathrm{~mL}$ \\
\hline & \\
\hline Energy density & $273.3659 \mathrm{Wh} / \mathrm{L}$ \\
\hline & \\
\hline U (BG formula) & $39.12 \%$ \\
\hline
\end{tabular}

$\mathrm{F}_{\mathrm{v}, \text { air }}=200 \mathrm{~mL} / \mathrm{min}$

Dose trigger $=100 \mathrm{mV}$

Temp $=60^{\circ} \mathrm{C}$

Figure 26c. IFDL DMFC operation in dose mode\#3 


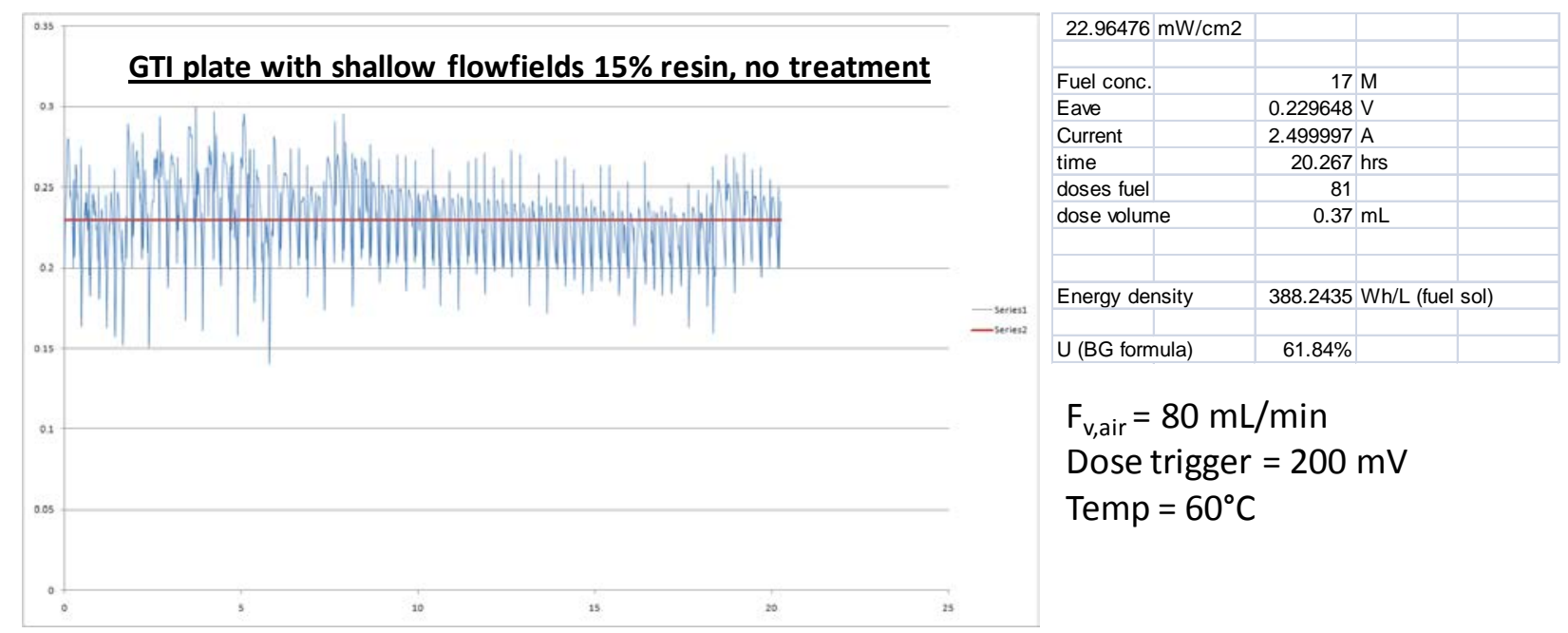

Figure 26d. IFDL DMFC operation in dose mode\#4

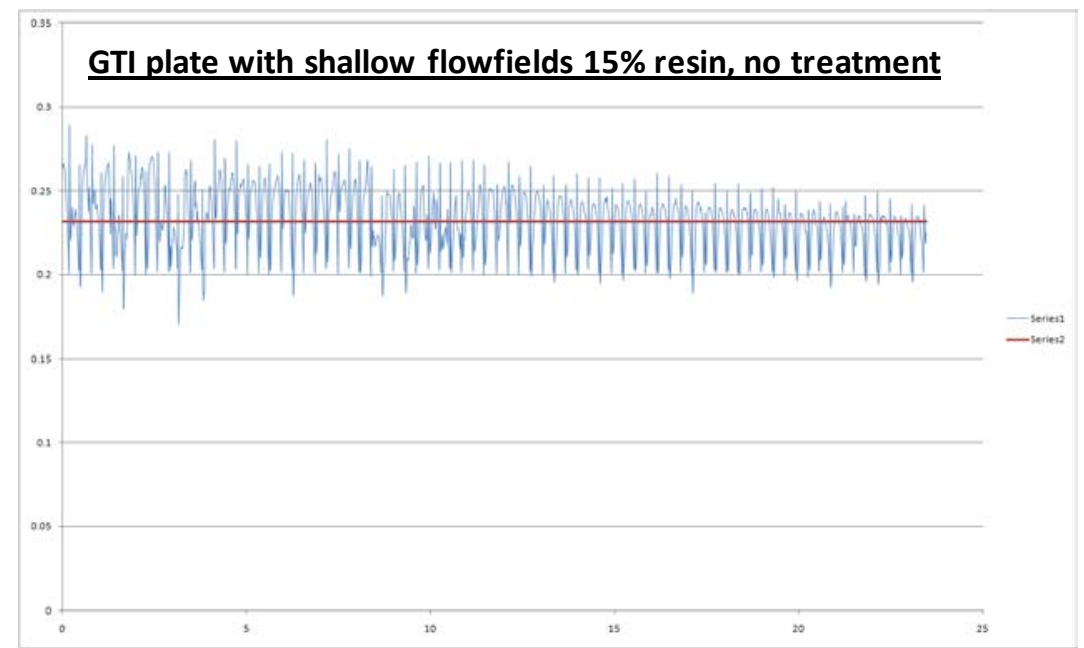

\begin{tabular}{|l|r|l|}
\hline 18.53382 & $\mathrm{~mW} / \mathrm{cm} 2$ & \\
\hline Fuel conc. & $17 \mathrm{M}$ \\
\hline Eave & $0.231673 \mathrm{~V}$ \\
\hline Current & $1.999999 \mathrm{~A}$ \\
\hline time & $23.45 \mathrm{hrs}$ \\
\hline doses fuel & 76 \\
\hline dose volume & $0.37 \mathrm{~mL}$ \\
\hline & & \\
\hline Energy density & $386.3959 \mathrm{Wh} / \mathrm{L}$ (fuel sol) \\
\hline & & \\
\hline U (BG formula) & $61.01 \%$ & \\
\hline
\end{tabular}

$\mathrm{F}_{\mathrm{v} \text {,air }}=60 \mathrm{~mL} / \mathrm{min}$

Dose trigger $=200 \mathrm{mV}$

Temp $=60^{\circ} \mathrm{C}$

Figure 26e. IFDL DMFC operation in dose mode\#5 

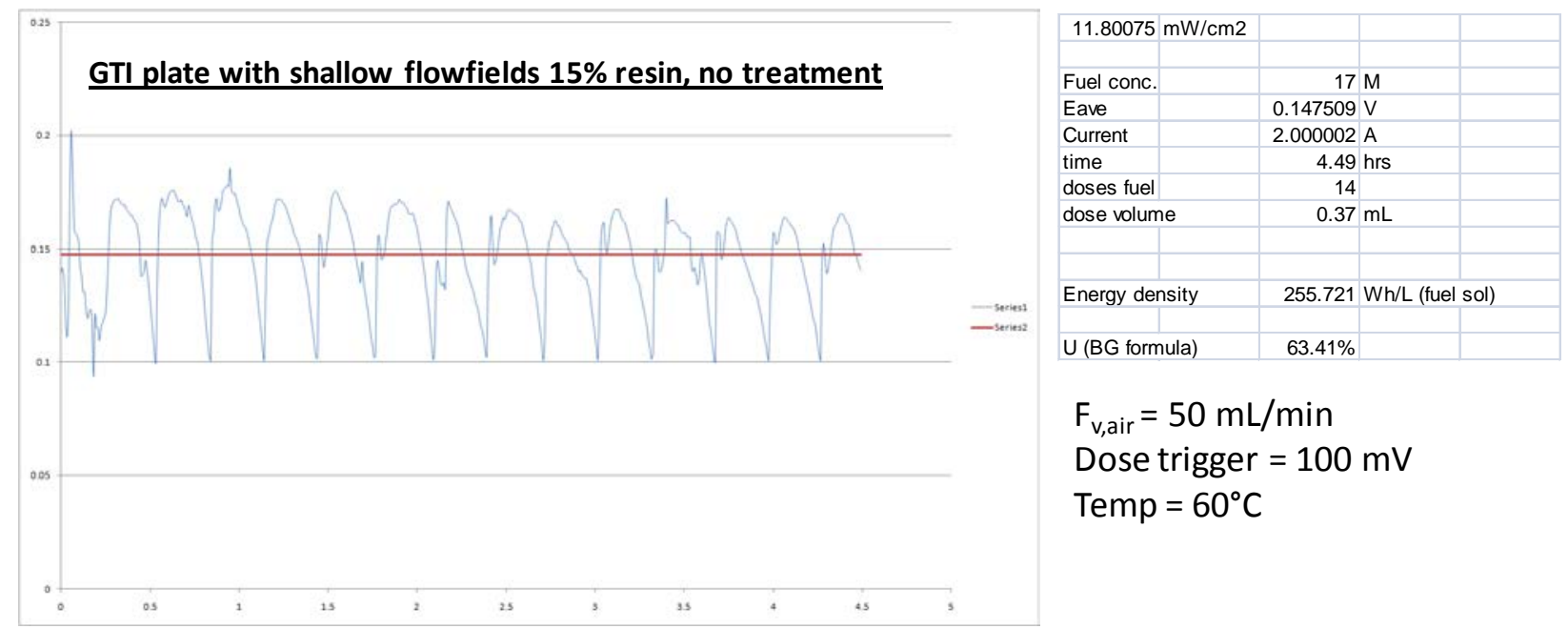

Figure 26f. IFDL DMFC operation in dose mode\#6

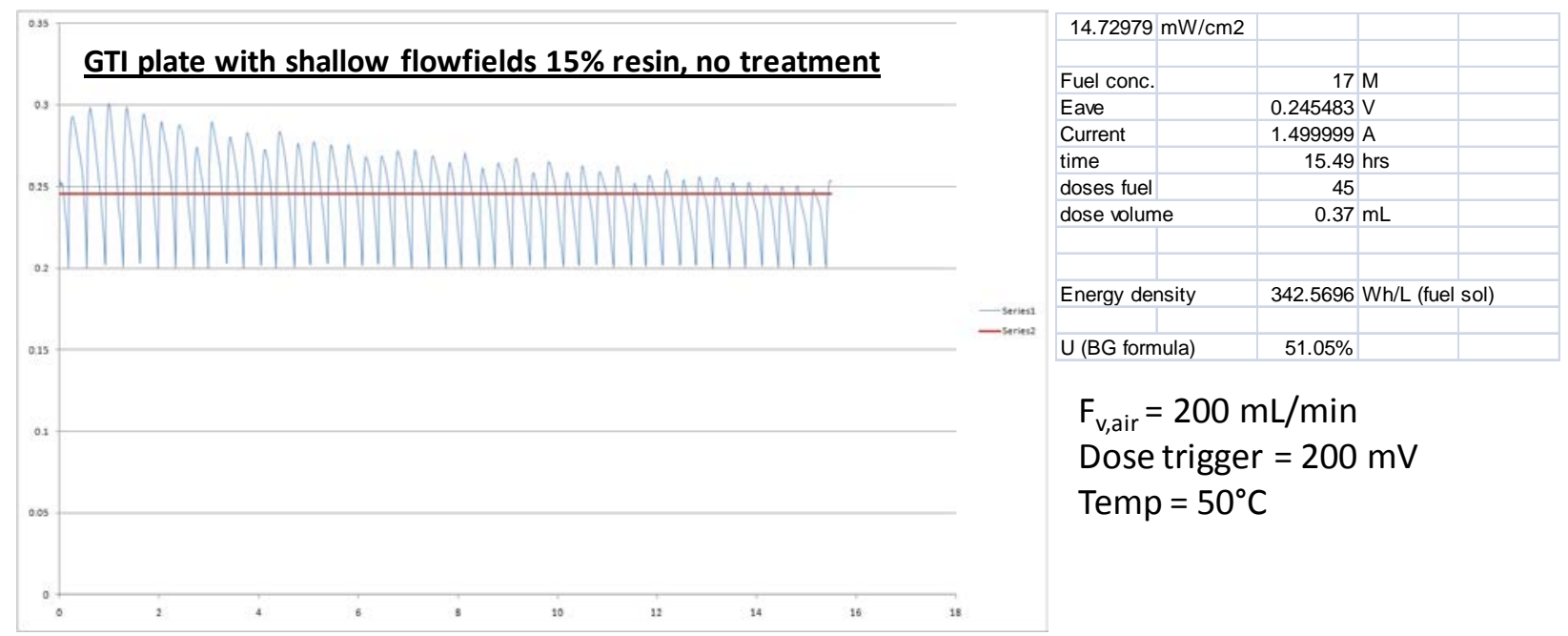

Figure 26g. IFDL DMFC operation in dose mode\#7

It can be easily seen from figures 26 a-g that the performance of the IFDL DMFC in all cases is not very good. The average cell voltage is low (below 0.3V) leading to low GFED (less than 400 $\mathrm{W}$ *hr/ $/ \mathrm{L}_{\text {fuel }}$ ) and poor fuel utilization. Other results with the IFDL DMFC operated in dosing mode are shown in table 6. 
Table 6. Summary of results with the IFDL DMFC operated in dosing mode

\begin{tabular}{|c|c|c|c|c|c|}
\hline $\begin{array}{c}\text { Operating Conditions } \\
\text { (Icell, } \mathrm{MeOH} \text { conc., } \mathrm{T}^{\circ} \mathrm{C} \text {, Air Flowrate) }\end{array}$ & $\begin{array}{c}\text { GFED } \\
\left(W^{*} h r / \text { Lfuel }\right)\end{array}$ & $\begin{array}{l}\text { Eavg } \\
\text { (V) }\end{array}$ & $\begin{array}{c}\text { Fuel Utilization } \\
\text { (\%) }\end{array}$ & $\frac{\text { Pavg }}{(\mathrm{mW} / \mathrm{cm} 2)}$ & $\frac{P \max }{(\mathrm{mW} / \mathrm{cm} 2)}$ \\
\hline $1 \mathrm{Amp}, 13 \mathrm{M}, 60^{\circ} \mathrm{C}, 200 \mathrm{SCCM}$ & 176 & 0.34 & 24 & 14 & 17 \\
\hline $1 \mathrm{Amp}, 13 \mathrm{M}, 60^{\circ} \mathrm{C}, 120 \mathrm{SCCM}$ & 313 & 0.38 & 39 & 15 & 18 \\
\hline $1 \mathrm{Amp}, 17 \mathrm{M}, 60^{\circ} \mathrm{C}, 200 \mathrm{SCCM}$ & 201 & 0.40 & 18 & 16 & 21 \\
\hline $1 \mathrm{Amp}, 17 \mathrm{M}, 60^{\circ} \mathrm{C}, 120 \mathrm{SCCM}$ & 267 & 0.41 & 24 & 16 & 19 \\
\hline 1.5 Amp, $13 \mathrm{M}, 60^{\circ} \mathrm{C}, 200 \mathrm{SCCM}$ & 234 & 0.34 & 33 & 20 & 23 \\
\hline $1.5 \mathrm{Amp}, 13 \mathrm{M}, 60^{\circ} \mathrm{C}, 120 \mathrm{SCCM}$ & 134 & 0.14 & 46 & 8 & 11 \\
\hline $1.5 \mathrm{Amp}, 17 \mathrm{M}, 60^{\circ} \mathrm{C}, 200 \mathrm{SCCM}$ & 260 & 0.38 & 25 & 23 & 26 \\
\hline $1.5 \mathrm{Amp}, 17 \mathrm{M}, 60^{\circ} \mathrm{C}, 120 \mathrm{SCCM}$ & 336 & 0.37 & 33 & 22 & 28 \\
\hline $1.5 \mathrm{Amp}, 17 \mathrm{M}, 50^{\circ} \mathrm{C}, 120 \mathrm{SCCM}$ & 302 & 0.20 & 56 & 12 & 19 \\
\hline $1.5 \mathrm{Amp}, 17 \mathrm{M}, 40^{\circ} \mathrm{C}, 120 \mathrm{SCCM}$ & 143 & 0.11 & 46 & 7 & 8 \\
\hline $1.5 \mathrm{Amp}, 17 \mathrm{M}, 60^{\circ} \mathrm{C}, 80 \mathrm{sCCM}$ & 318 & 0.28 & 41 & 17 & 23 \\
\hline $2 \mathrm{Amp}, 13 \mathrm{M}, 60^{\circ} \mathrm{C}, 200 \mathrm{SCCM}$ & 262 & 0.29 & 44 & 23 & 28 \\
\hline $2 \mathrm{Amp}, 17 \mathrm{M}, 60^{\circ} \mathrm{C}, 120 \mathrm{SCCM}$ & 352 & 0.30 & 42 & 24 & 32 \\
\hline $2 \mathrm{Amp}, 17 \mathrm{M}, 60^{\circ} \mathrm{C}, 80 \mathrm{SCCM}$ & 124 & 0.13 & 35 & 10 & 20 \\
\hline
\end{tabular}

It appears the dosing mode is not a good method to operate the IFDL DMFC.

\section{Continuous fuel delivery mode}

This operation requires the availability of a high precision, low flowrate pump. We have used an ISCO pump capable of delivering flowrates with nano liter precision. The fuel is delivered continuously at $\mu$ Liter/min flowrates at values slightly in excess of the stoichiometric ratio dictated by the operating current.

The IFDL DMFCs are operated at constant current over prolonged periods of time and, as in the case with dosed fuel, the average voltage, average power, GFED and fuel utilization are calculated.

Several examples with various MEAs operated with this type of fuel delivery, together with the operating conditions are given in figures 27 a-g. 


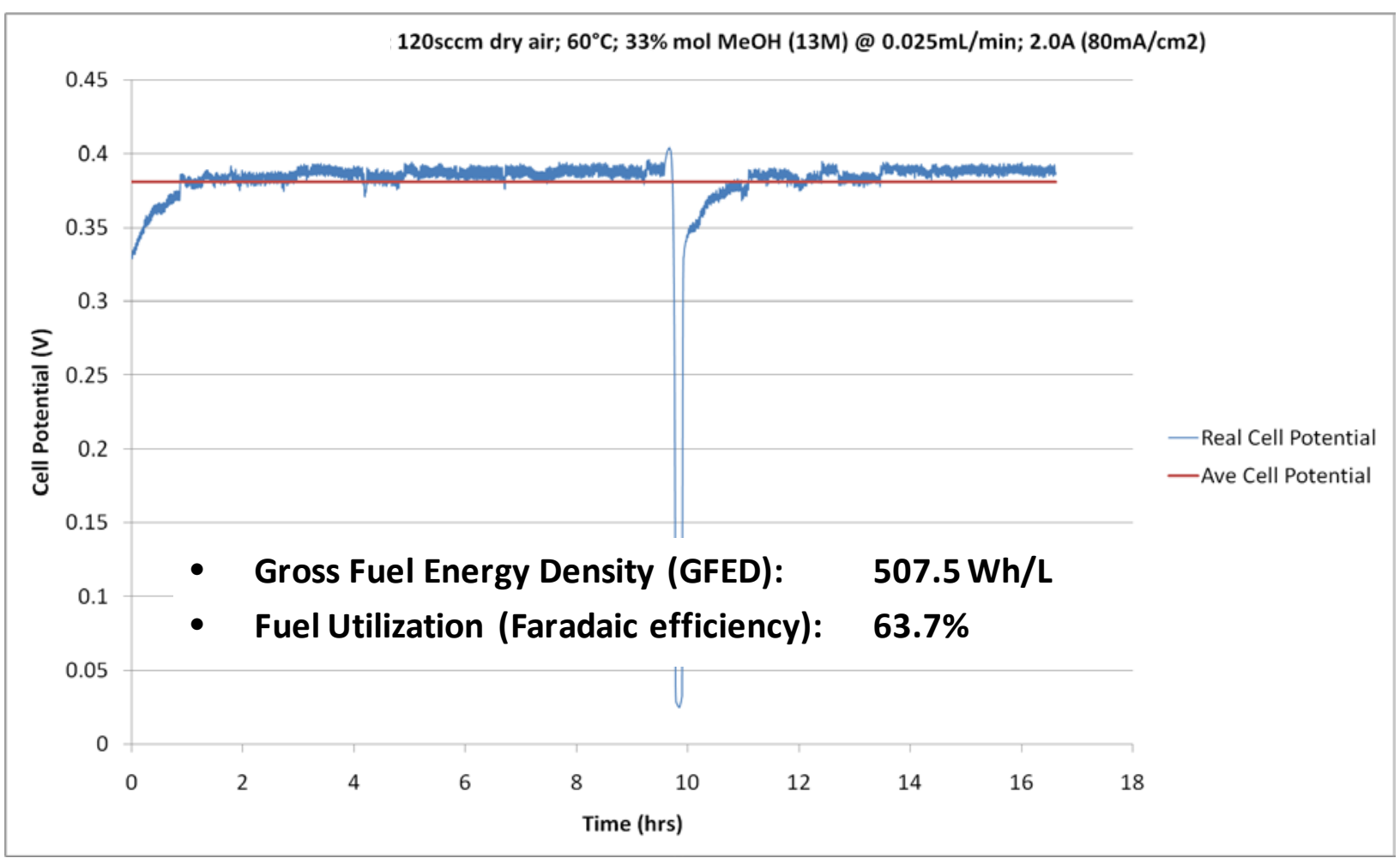

Figure 27a. IFDL DMFC operation in continuous mode\#1

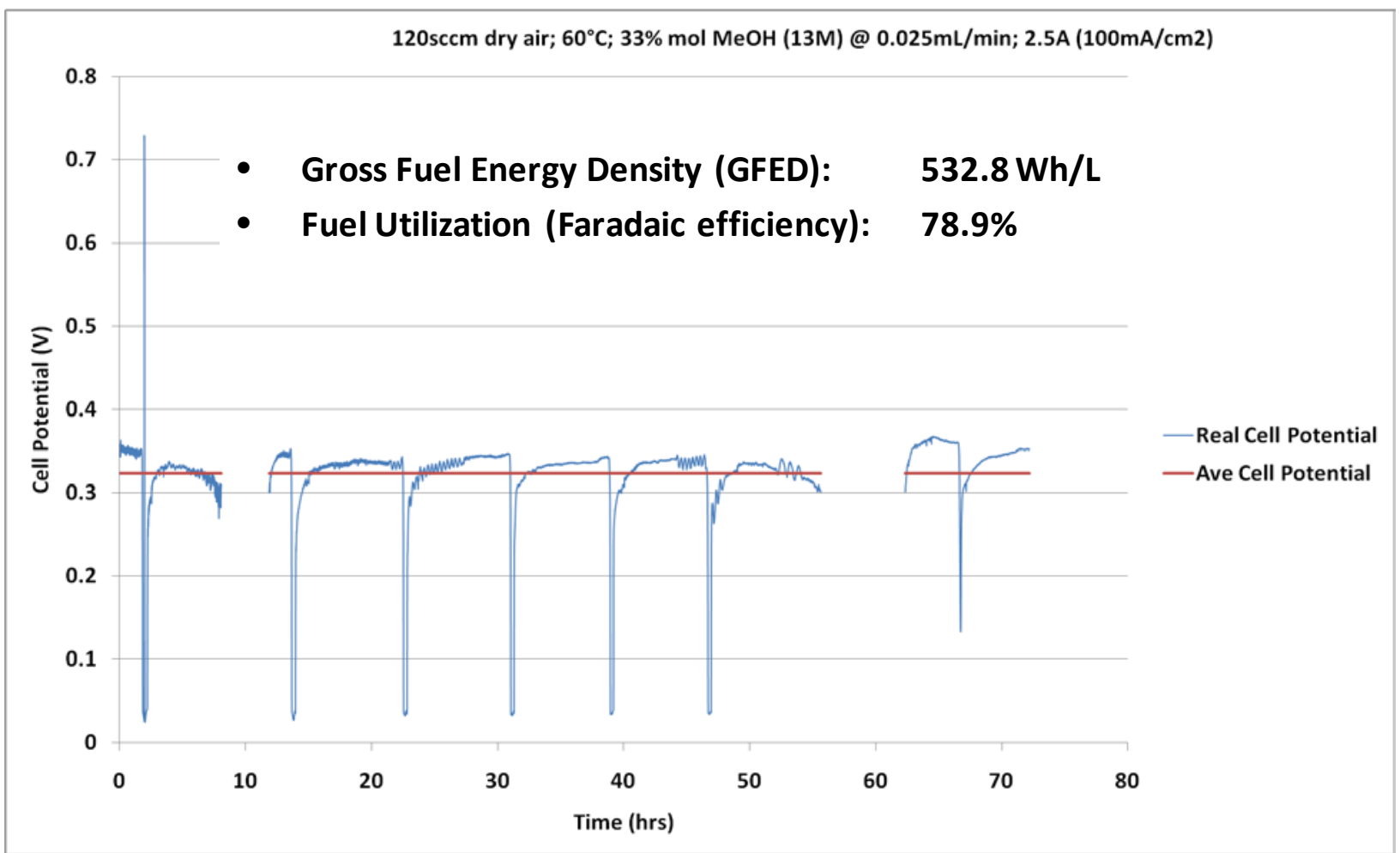

Figure 27b. IFDL DMFC operation in continuous mode\#2 


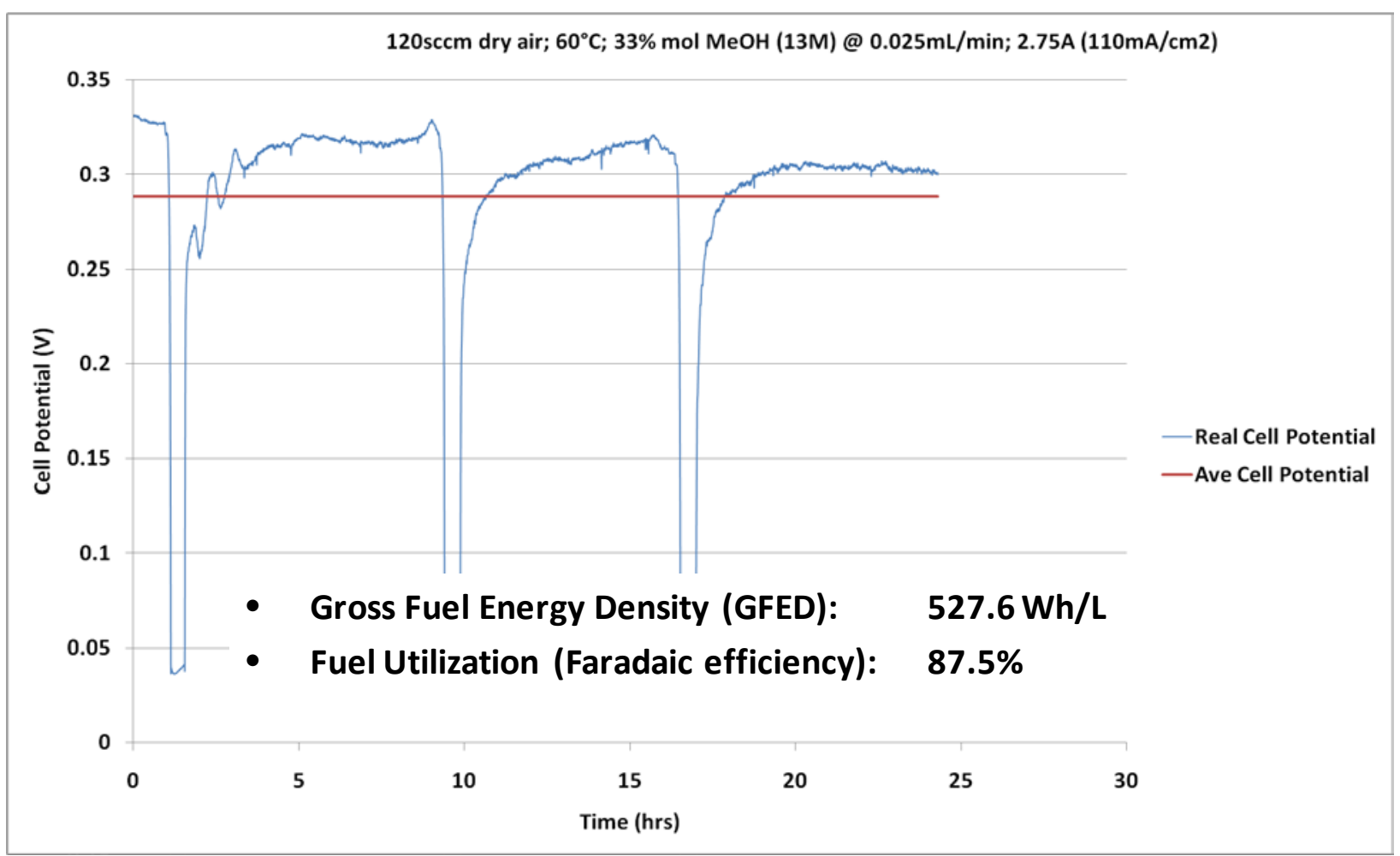

Figure 27c. IFDL DMFC operation in continuous mode\#3

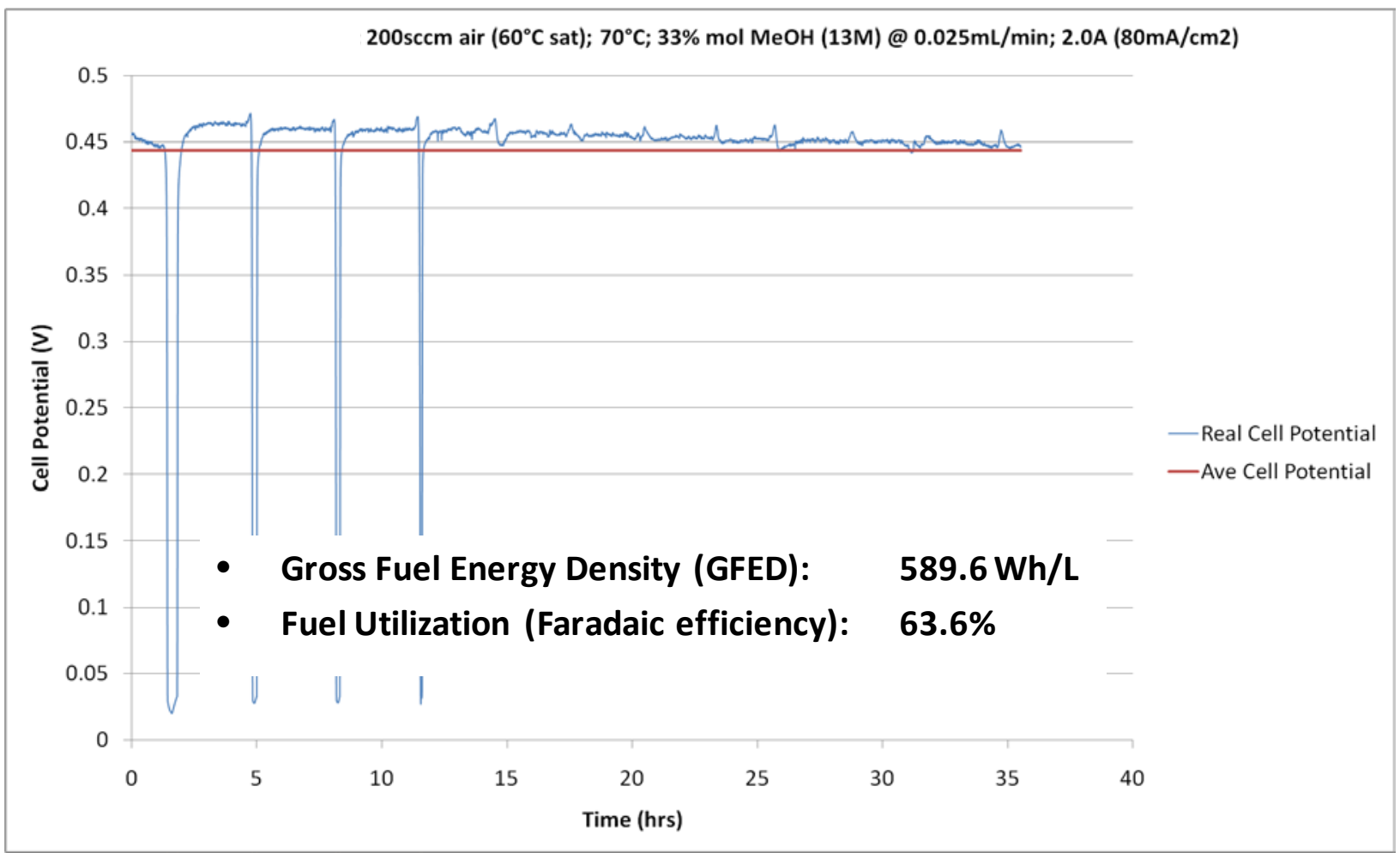

Figure 27d. IFDL DMFC operation in continuous mode\#4 


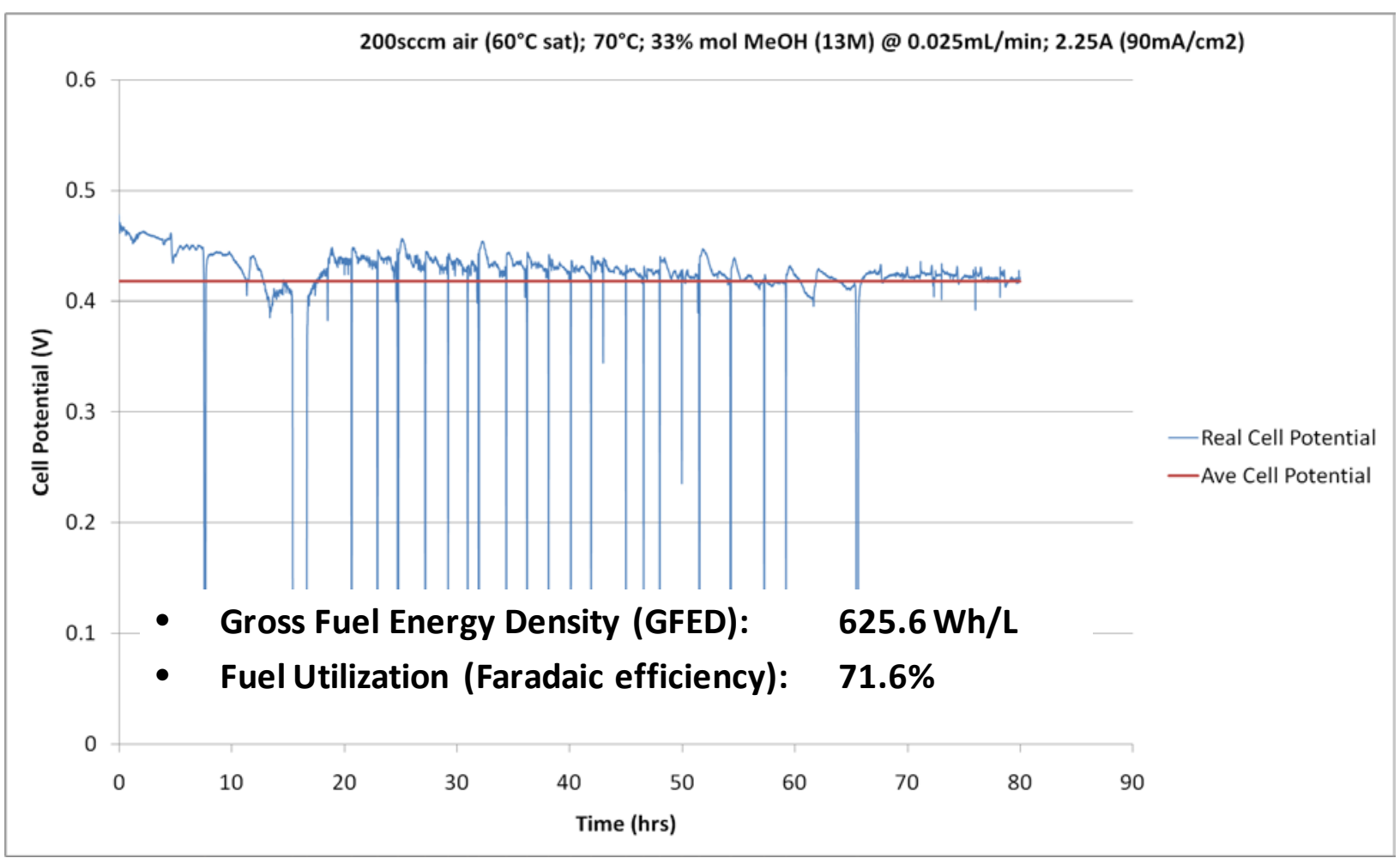

Figure 27e. IFDL DMFC operation in continuous mode\#5

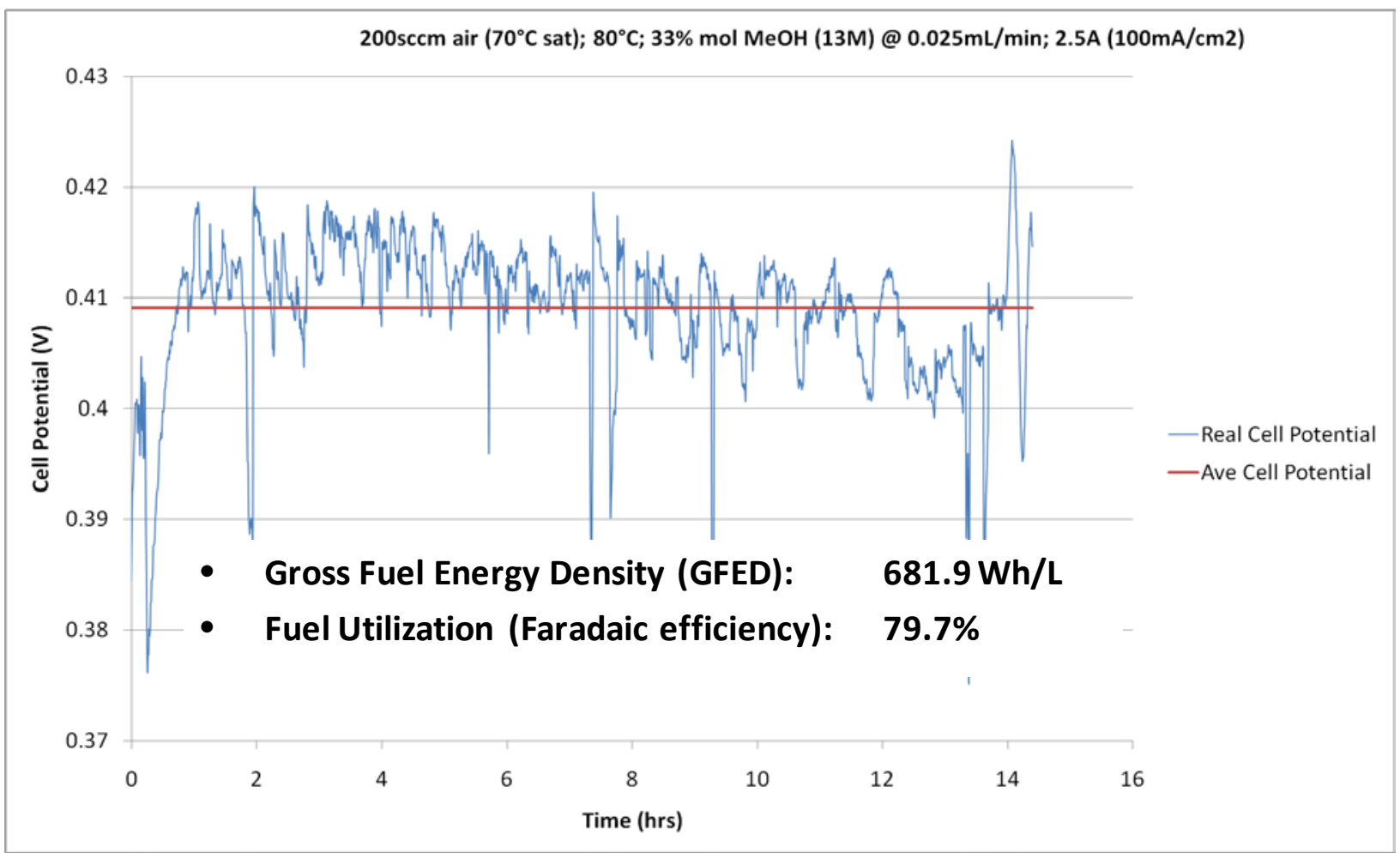

Figure 27f. IFDL DMFC operation in continuous mode\#6 


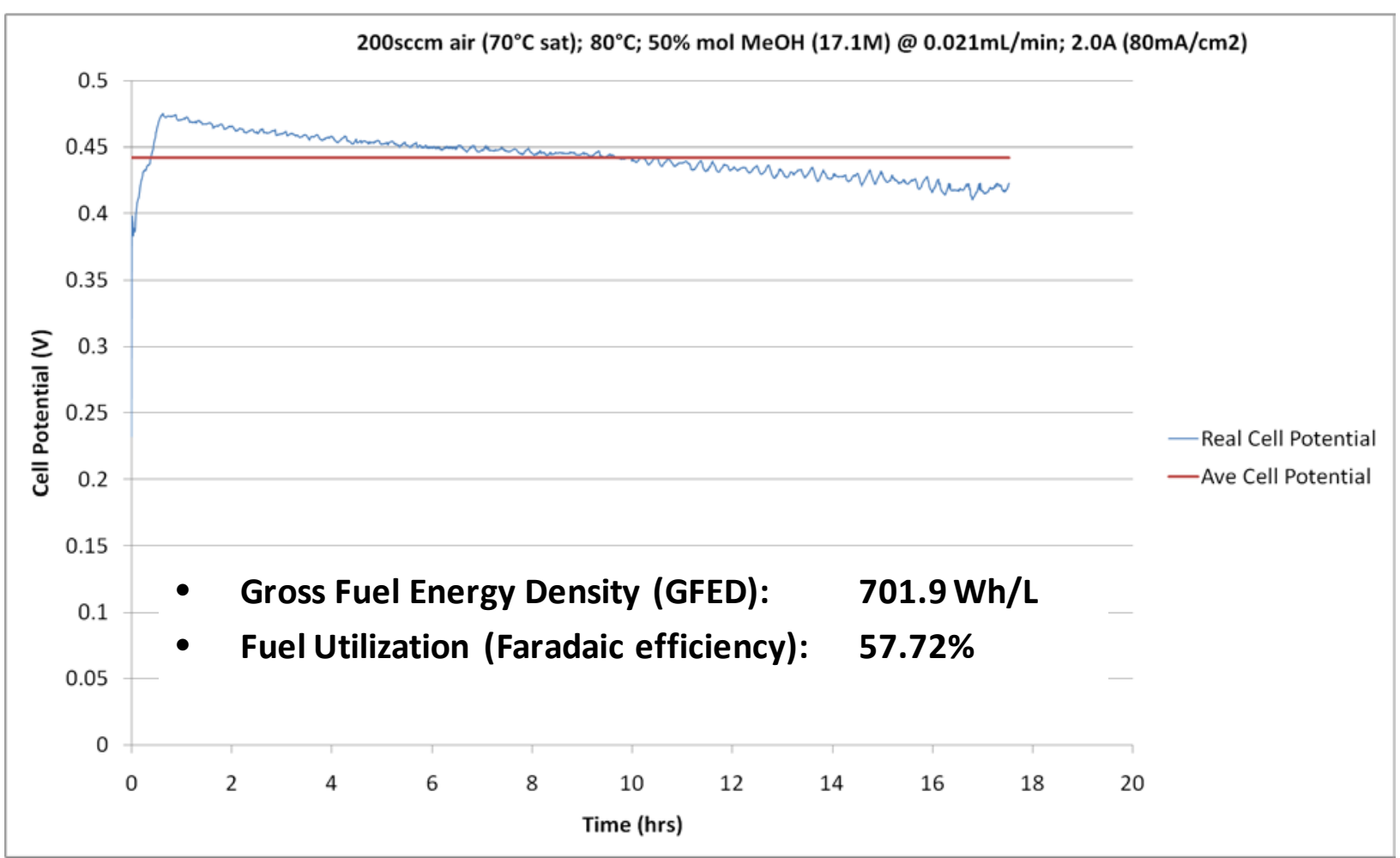

Figure 27g. IFDL DMFC operation in continuous mode\#7

It can be easily noticed that the performance improved significantly with both the average cell voltage and fuel utilization increasing. The cells were operated in constant feed mode at 0.025 $\mathrm{mL} / \mathrm{min}$. When the flowrate was dropped to $0.021 \mathrm{~mL} / \mathrm{min}$ the gross fuel energy density exceeded $700 \mathrm{Wh} /$ liter. The gross fuel energy density increased from 352 in the case of dosed fuel delivery to over $700 \mathrm{Wh} / \mathrm{L}$ in the case of continuous fuel delivery which is a significant increase. Fuel utilization increased from $42 \%$ to over $70 \%$ in some cases.

In some of the MEAs that were tested we have noticed intermittent voltage drops. The cause for these drops is still unclear but we suspect that the main reason was either one (or both) of the following:

- water accumulation flooding the cathode. As water builds in the cathode it blocks the access of air to the catalyst which, in turn, causes the voltage to drop. When enough water accumulates and creates internal pressure it shoots out relieving the cathode of flooding and recovering the performance

- gas accumulation in the anode. As methanol is oxidized at the anode it releases carbon dioxide which (in spite of the evacuation conduits) blocks the diffusion of fresh methanol to the anode catalyst. When a sufficiently large amount of carbon dioxide has 
accumulated it shoots out under the internal pressure freeing the path for fresh methanol to enter the catalyst layer.

We have continued to optimize the operation of our better MEAs and gradual improvement was achieved.

Our best performance is shown in figure $28 a-b$.

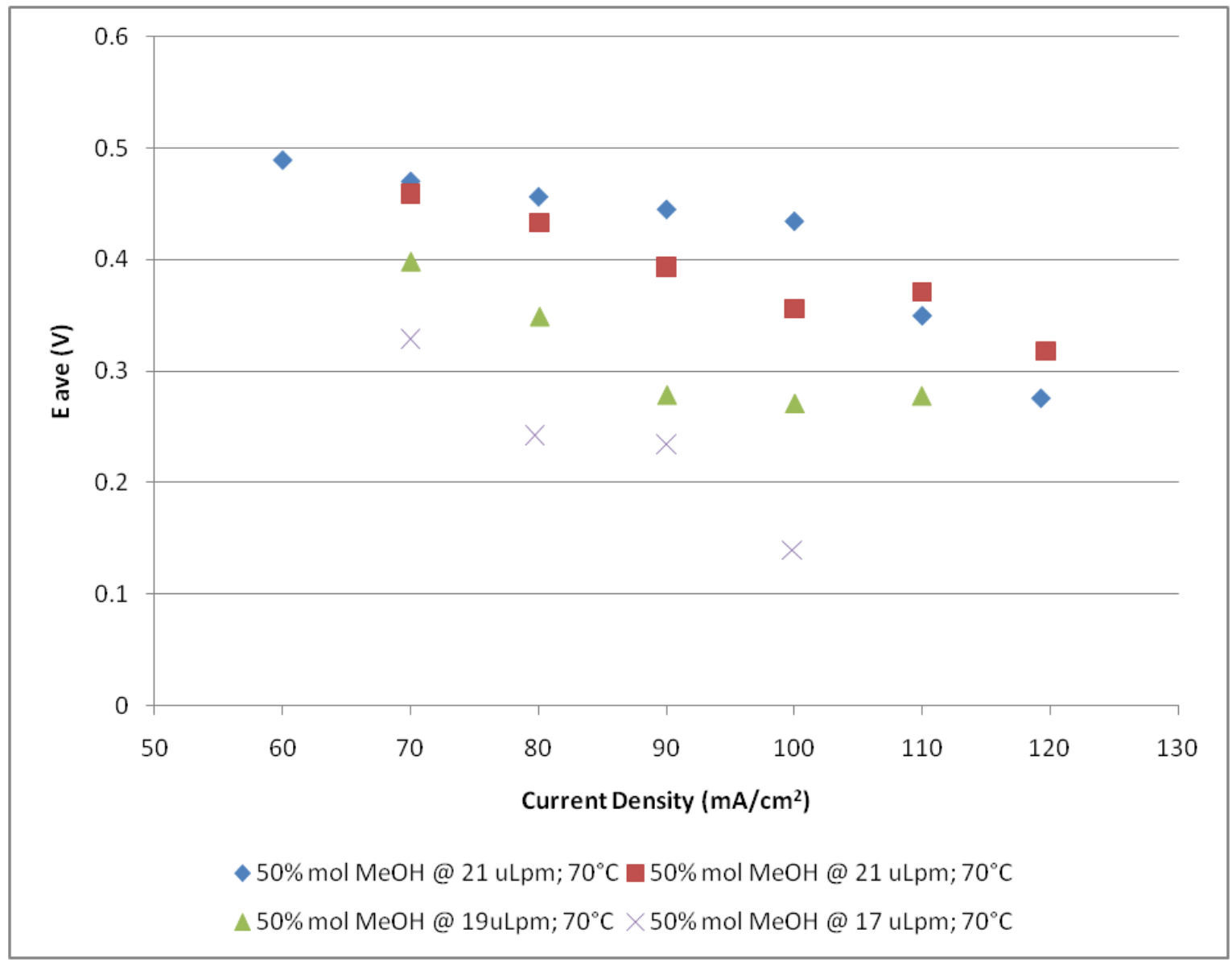

Figure 28a. Best IFDL DMFC performance achieved 


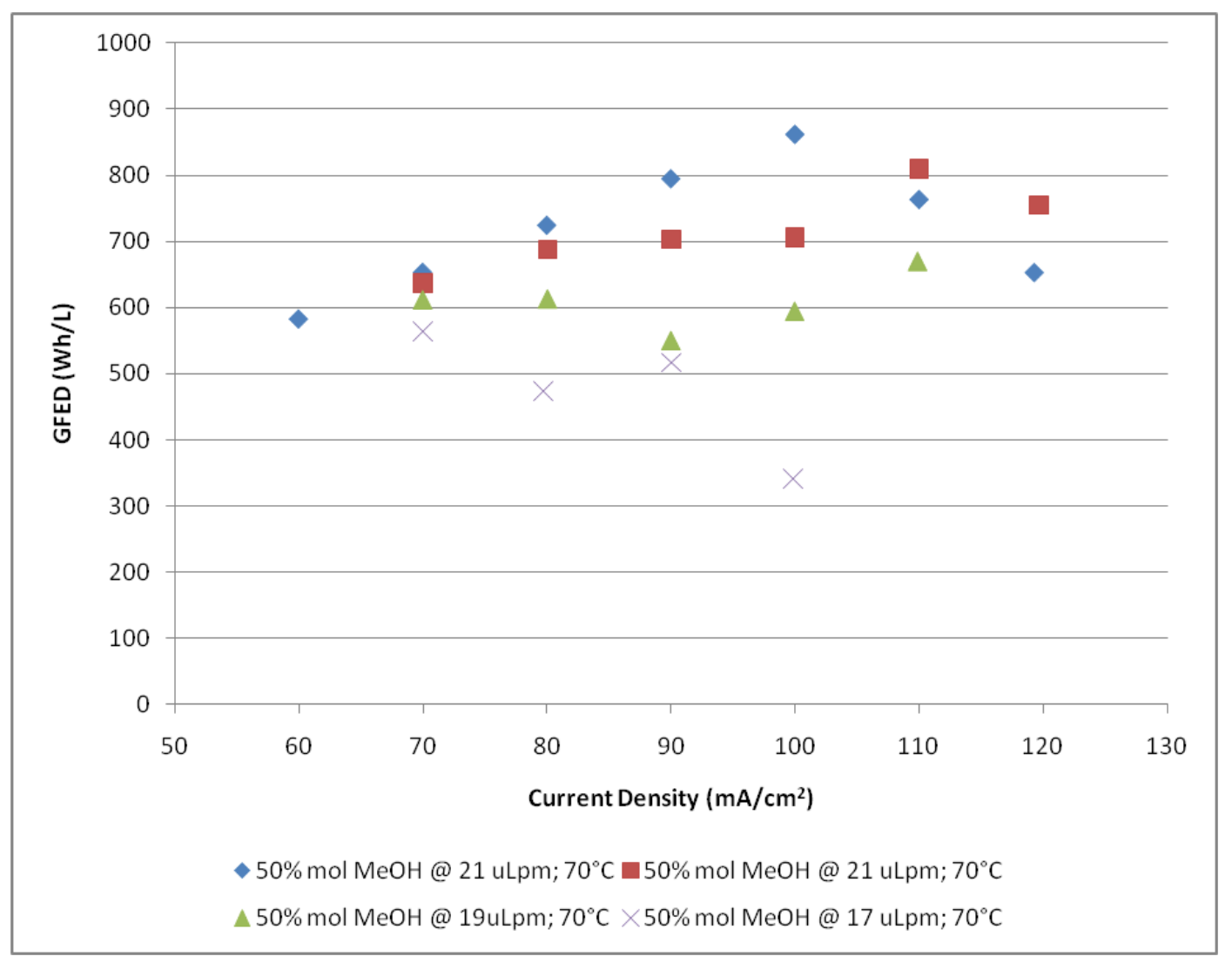

Figure 28b. Best GFED

It can be easily noticed that the GFED is nearing $900 \mathrm{Wh} / \mathrm{L}$ which is an exceptionally good performance for a fuel cell of this type.

Most of the fuel cell systems that are reported in the literature are orientation dependent. This means that they can only be operated in a fixed position. Achieving operation independence is very important for portable power sources, as many of them need to be used in the field where they cannot always be placed in the manufacturer's intended position.

To assess the impact that orientation has on our fuel cell, we have operated our IFDL DMFC in an upward position and then placed it on its side and repeated the experiment. The results are shown in figure 29: 


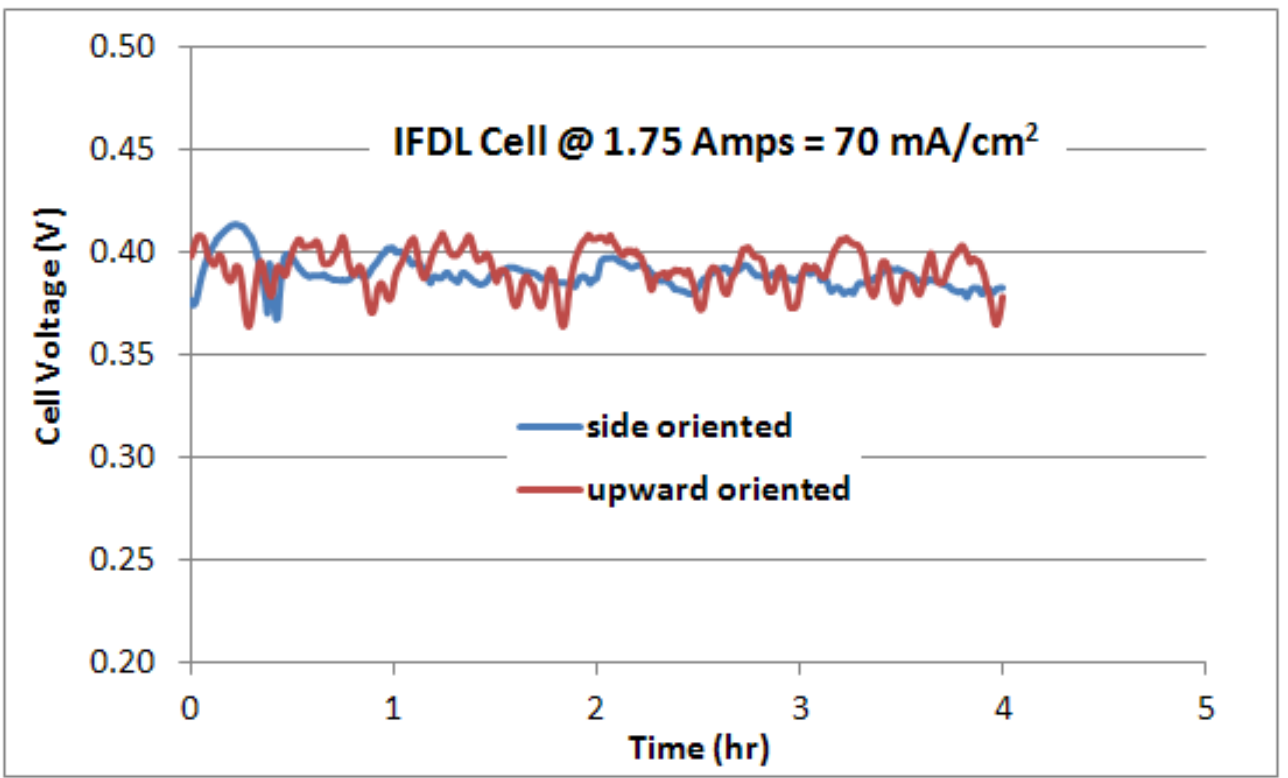

Figure 29. Assessment of orientation dependence of the IFDL DMFC

The cell voltage appears to be stable regardless of whether the fuel cell is operated in vertical or horizontal position.

We have proven that IFDL DMFCs can deliver high GFED, high fuel utilization and can be operated for extended periods of time orientation independent. 


\section{Milestone\#4, Build a research-grade short stack.}

We have built a short 2 cell stack that accommodates NuVant's technology of operation of IFDL DMFCs with elevated methanol concentrations. The constituent parts of the stack as well as the assembled stack are shown in figures 30 and 31.

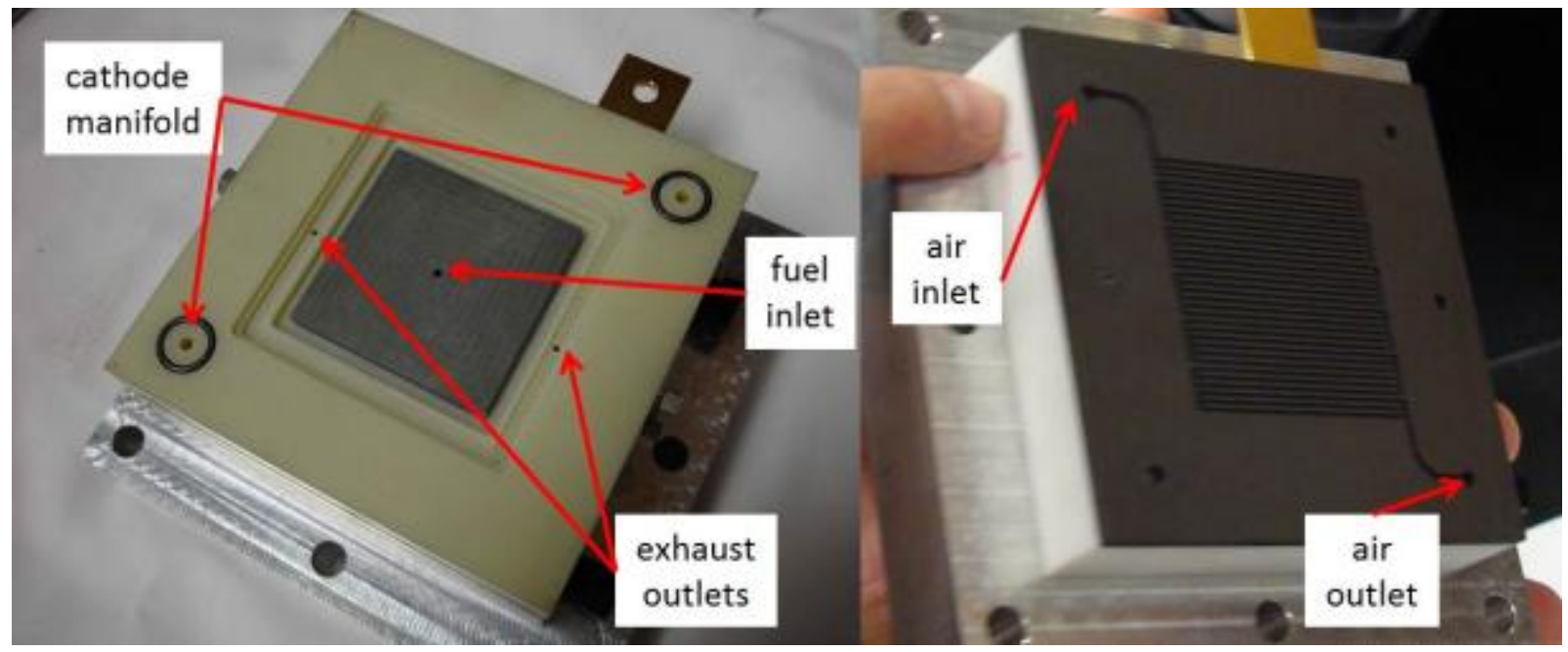

Figure 30. Exploded view of main stack constituents. Anode housing without the porous plate (left). Cathode flowfield (right)

The porous plate is lodged inside the anode housing which is shown without the porous plate in figure 30. The fuel enters through the middle hole, diffuses through the porous structure and reacts at the anode. The exhaust gas exits the anode housing through the 2 exhaust holes shown in the picture (left). The cathode plate is comprised of a conventional serpentine flowfield and is shown at the right in figure 30 . While the fuel enters the anode plates independently, the air enters the cathode flow field via an internal manifold.

NuVant fabricated MEAs are placed in between the two plates shown in figure 30 via additional gaskets. A view of the assembled stack is shown in figure 31.

The stack was operated with $17 \mathrm{M} \mathrm{MeOH}$ solution and $400 \mathrm{sccm}$ of air. 


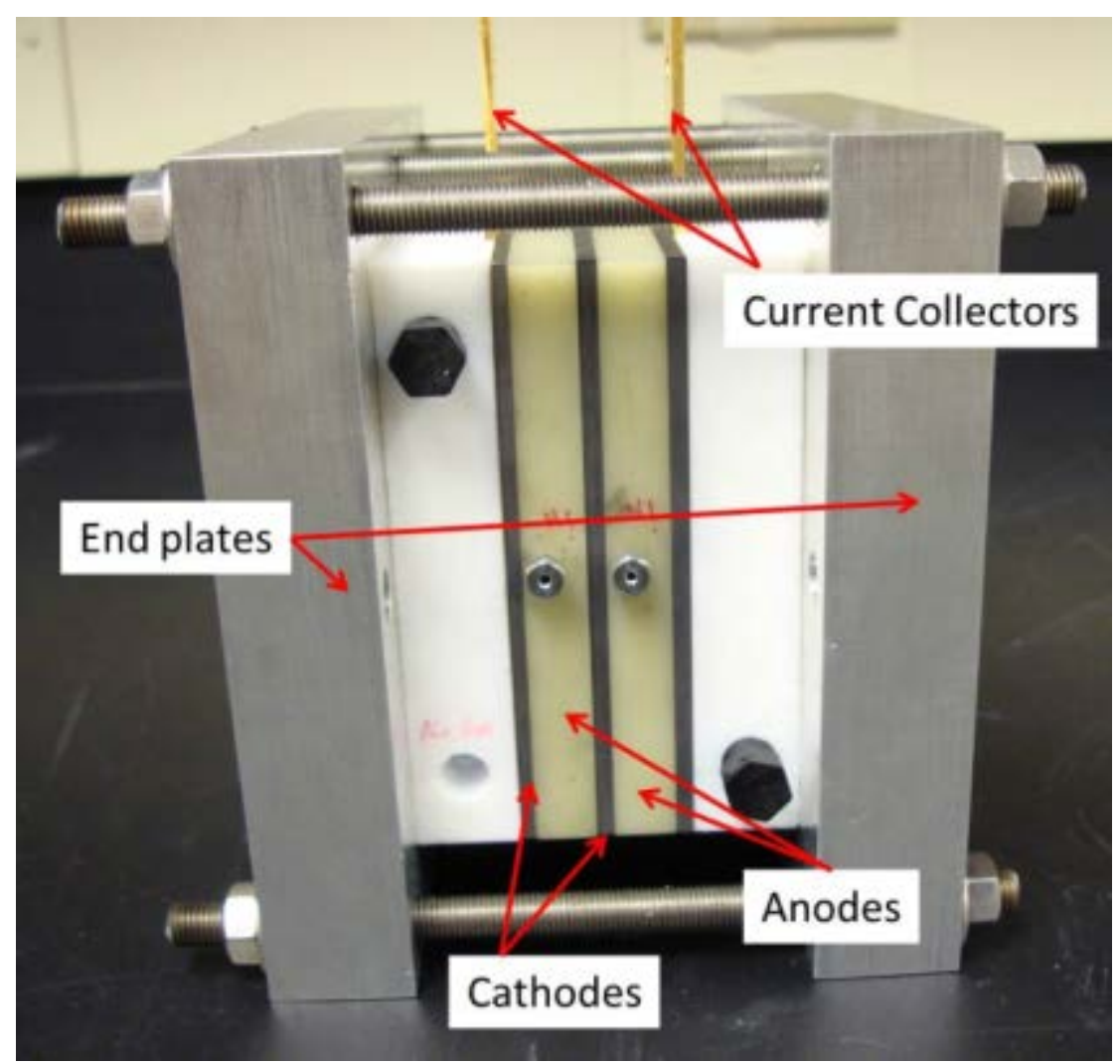

Figure 31. Assembled stack

A preliminary polarization curve is shown in figure 32 .

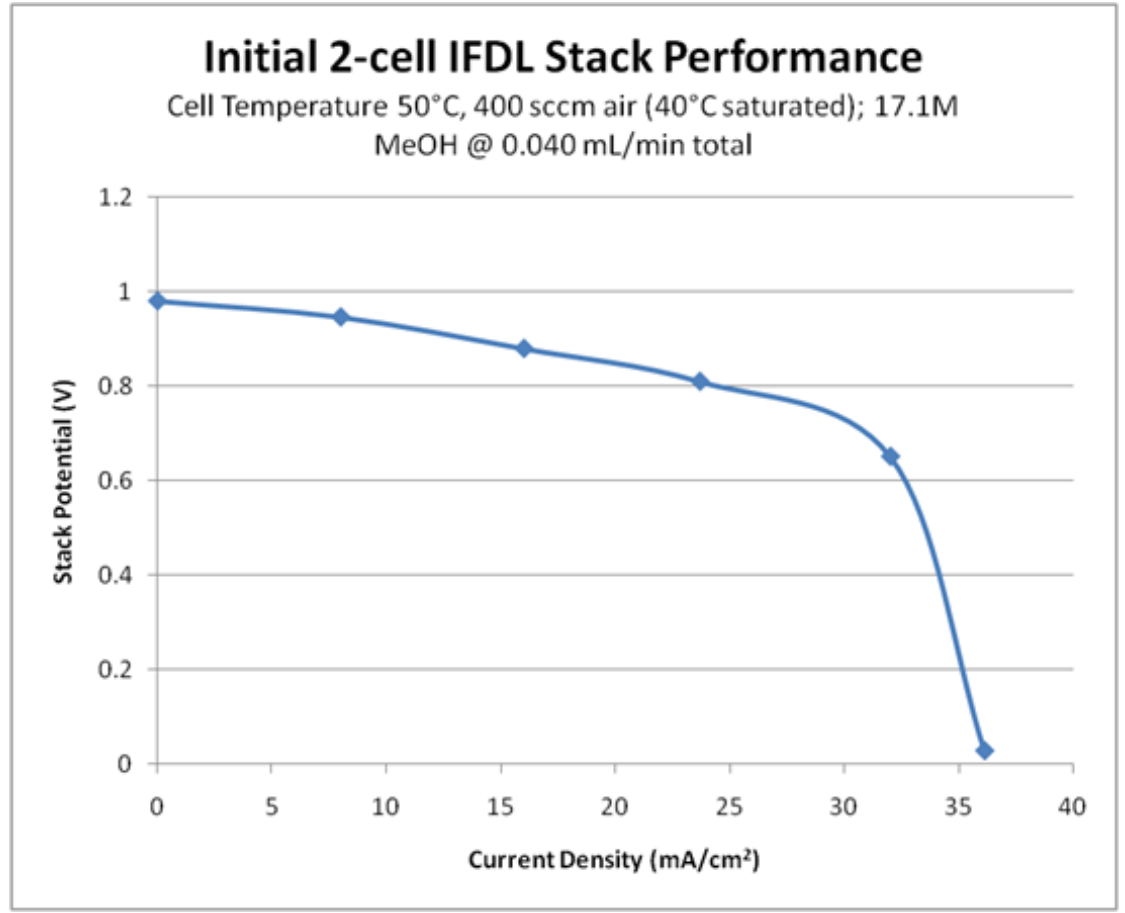

Figure 32. Preliminary performance of the short DMFC stack 
Unfortunately, one of the 2 MEAs assembled inside of the DMFC stack did not perform very well and created the large mass transport drop around the $30 \mathrm{~mA} / \mathrm{cm}^{2}$ mark. The MEA was replaced and the performance was re-evaluated.

The result with a proper MEA replacing the faulty one is shown in figure 33 .

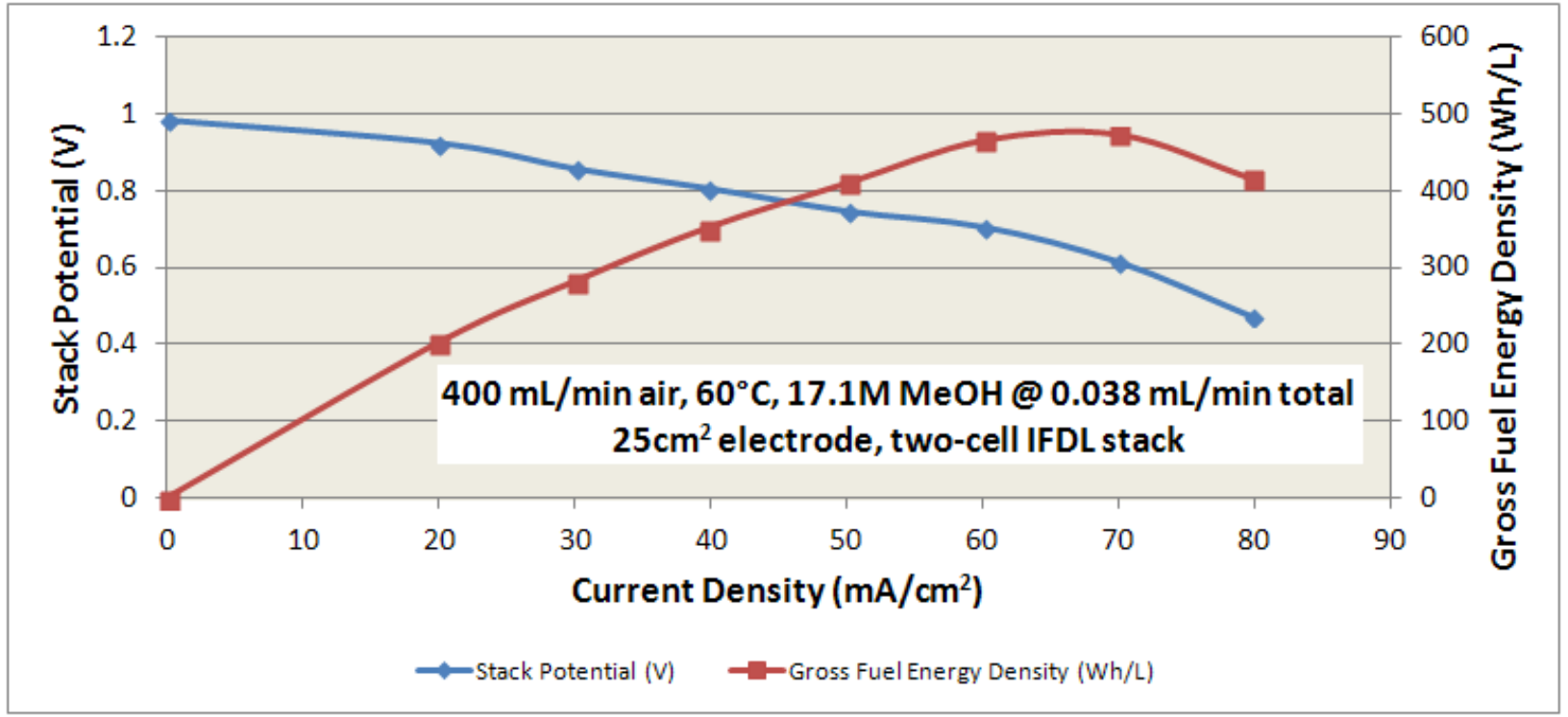

Figure 33. IFDL DMFC 2-cell stack performance

The performance improved tremendously compared with the first stack results but there still is some mass transport effects around maximum GFED which could probably improve even further.

This results proves that the IFDL concept can be extrapolated to more than one cell and very likely to larger electrode area than the $25 \mathrm{~cm}^{2}$ that was employed throughout of this project. 


\section{Milestone\#5. Perform additional modeling to account for transport of species within the cell and electrochemical reaction.}

It had become obvious that only modeling the flow through the porous plate in order to assess the rate of delivery of fuel to the anode catalyst would not suffice in building a model of the entire IFDL DMFC that can be verified against experimental values. The transport of species through all the IFDL DMFC interfaces needs to be accounted for as well as species consumption/production via electrochemical reactions.

A simple 2D isothermal single-phase Direct Methanol Fuel Cell model has been created to investigate the transport of species and the influence of species concentration on cell performance. The specific scope of the model is to simulate the transport and generation of liquid water and water vapor and study its transport from the cathode to the anode side of the cell creating dilution of the liquid methanol fuel.

\section{Model Geometry and Assumptions}

The computational domain contains the IFDL, anode and cathode diffusion layers, catalyst layers, and membrane. The model does not contain flow channels (open flow regions) on the cathode or anode side of the cell or contain free flow in the anode flow channels created by the IFDL. The anode reactants (water and methanol) uniformly enter the left side of the cell as a well-mixed binary species. The cathode reactants enter the top section of the cathode GDL and exit the bottom. The indentation that is present in the cathode gas diffusion layer is to account for compression from the flow channel rib. The open channels in the IFDL to allow for carbon dioxide removal are not used in the model.

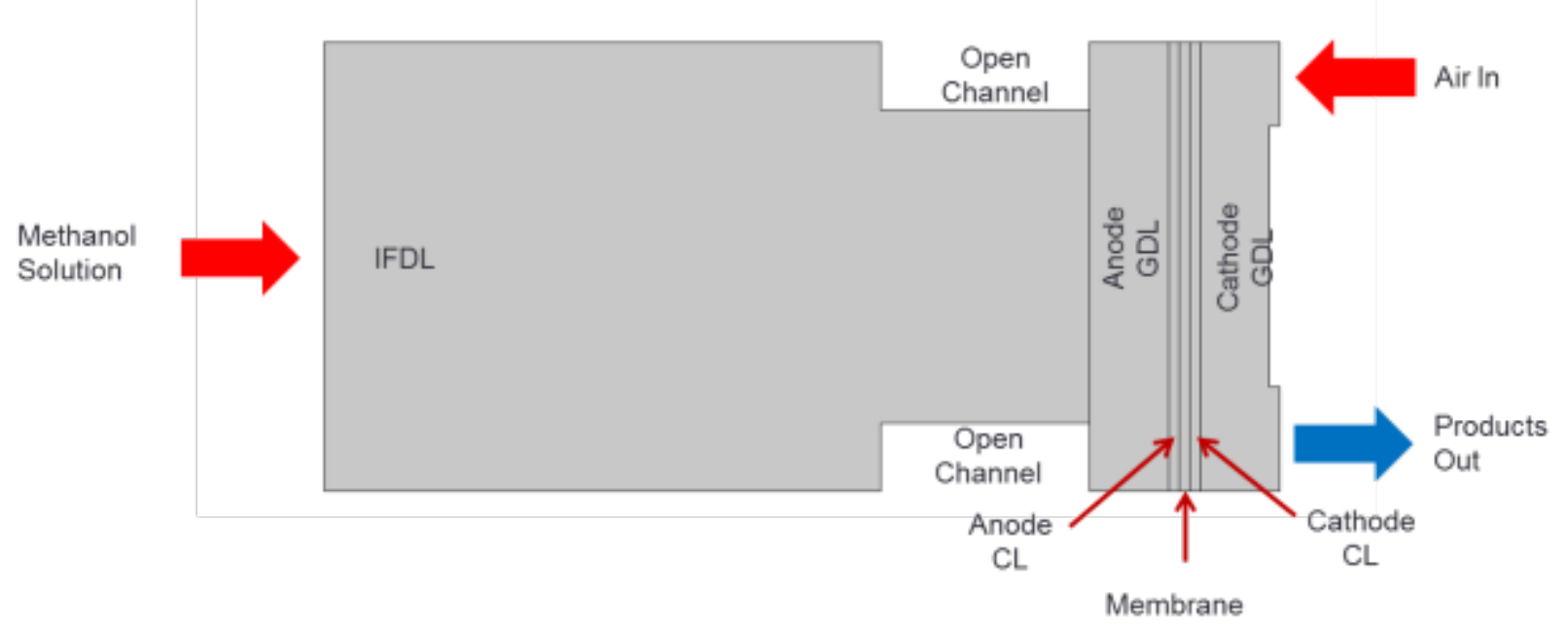


Figure 34: DMFC model geometry used for simulation

It should be noted that the model uses uniform catalyst layers (instead of two separate ones places together).

Model Assumptions

A list of the major assumptions used for the model is shown below:

Production and transport of $\mathrm{CO} 2$ is neglected

All domains are isothermal and at the same uniform temperature

The fuel cell operates under steady state conditions

The concentration of methanol is kept constant at the anode inlet

The concentration of oxygen and water vapor is kept constant at the cathode inlet

All species are well mixed

Methanol-water mixture on the anode is an incompressible liquid

Water vapor, oxygen, and nitrogen in the cathode of the GDL and CL exist as mixed ideal gases

Water transported through the membrane to the cathode is instantly vaporized

The methanol oxidation reaction at the cathode and membrane interface is instantaneous (no methanol is contained in the cathode catalyst domain)

All species are incompressible

Computational Model and Methodology

Diffusion Layers:

The anode and cathode diffusion layers as well as the IFDL share the same equations for convective mass transport. Convective transport for both liquid and gaseous species is modeled using the Brinkman-Stokes Flow (1) for porous media which is shown in the equation below.

$\nabla \cdot\left[-\mathrm{pl}+\frac{\mu}{\epsilon_{\mathrm{p}}}\left(\nabla \mathrm{u}+(\nabla \mathrm{u})^{\mathrm{T}}\right)-\frac{2 \mu}{3 \epsilon_{\mathrm{p}}}(\nabla \cdot \mathrm{u}) \mathrm{l}\right]-\left(\frac{\mu}{\kappa_{\mathrm{br}}}+\beta|\mathrm{u}|+\mathrm{Q}_{\mathrm{br}}\right) \mathrm{u}+\mathrm{F}=0$

Also used for convective transport are conservations of mass (2). It assumed that density of the cathode reactant gases remains constant. 
$\rho \nabla \cdot u=0$

IFDL and Anode GDL: The IFDL and anode GDL are modeled as single domains of uniform porosity and permeability. The IFDL contains a single inlet for neat methanol and shares a boundary for diffusion of methanol and water to the anode GDL. The IFDL geometry also contains channels which are to be coated with PTFE. Currently, the model treats these PTFE coated surfaces as insulated walls where no species transport takes place. The channels themselves which are for $\mathrm{CO} 2$ removal are currently void of any mass transport.

Species transport is modeled using as Fick-type convective diffusion which is coupled with the Brinkman-Stokes equation.

$\mathrm{N}_{\mathrm{j}}=-\mathrm{D}_{\mathrm{i}, \mathrm{eff}} \nabla \mathrm{c}_{\mathrm{i}}+\mathrm{uc}_{\mathrm{i}}$

$D_{i, e f f}=\epsilon^{\tau} D_{i}$

Species transport is accounted for using the Conservation of Species equation (5).

$\nabla \cdot\left(-D_{i, e f f} \nabla c_{i}\right)+u \cdot \nabla c_{i}=R_{i}$

Charge or electron transport is modeled using Conservation of Charge (6) and Ohm's Law (7).

$\nabla \cdot j_{s}=Q_{s}$

$\mathrm{j}_{\mathrm{s}}=-\sigma_{\mathrm{s}} \nabla \emptyset_{\mathrm{s}}$

Cathode GDL: The cathode GDL has a structure symmetric of the anode GDL, however it includes an inlet (top) and outlet (bottom) boundary conditions. Humidified ideal air (O2, N2, and $\mathrm{H} 2 \mathrm{O}$ ideal gas mixture) is assumed at the inlet. The gases are discharged from the GDL to the channel at 0 Psig. The transport of liquid species is modeled using Stephan-Maxwell Diffusion (8). Hydrophobicity or cathode flooding is accounted for by modification to the effective diffusion coefficient, Deff, and/or the tortuosity, т. Conservation of Species, Charge, and Ohm's Law are used in this region as well.

$\mathrm{j}_{\mathrm{i}}=-\left(\rho \omega_{\mathrm{i}} \sum \mathrm{D}_{\mathrm{ik}, \mathrm{eff}} \mathrm{d}_{\mathrm{k}}\right)$ 


$$
\mathrm{d}_{\mathrm{k}}=\nabla \mathrm{x}_{\mathrm{k}}+\frac{1}{\mathrm{p}}\left[\left(\mathrm{x}_{\mathrm{k}}-\omega_{\mathrm{k}}\right) \nabla \mathrm{p}\right]
$$

Catalyst Layers:

Anode Catalyst Layer: The anode catalyst layer is modeled as a 2D domain using the same species and charge transport equations as the anode GDL (Convective Fick-type Diffusion). Methanol and water consumption from the oxidation-reduction reaction as well as electroosmotic drag of both species are accounted for in this region. The electro-kinetics are modeled using the concentration dependent Butler-Volmer equation (9).

$\mathrm{j}=\mathrm{j}_{\mathrm{O}, \mathrm{a}}\left(\frac{\mathrm{C}_{\mathrm{H} 2 \mathrm{O}}}{\mathrm{C}_{\mathrm{H} 2 \mathrm{O}, \text { ref }}}\right)^{0.5}\left(\frac{\mathrm{C}_{\mathrm{MeOH}}}{\mathrm{C}_{\mathrm{MeOH}, \mathrm{ref}}}\right)^{0.5} \exp \left[\frac{\alpha \mathrm{F \eta}}{\mathrm{RT}}\right]$

The exchange current density, jo,a, is determined using an experimental correlation (10) which is only temperature dependent.

$\mathrm{j}_{\mathrm{o}, \mathrm{a}}=94.25 \exp \left[\frac{33570}{\mathrm{R}}\left(\frac{1}{353}-\frac{1}{\mathrm{~T}}\right)\right]$

To account for electro-osmotic drag, species which are consumed by EOD are consumed along with species from the redox reaction by modifying the stoichiometric coefficients for the reaction. For example, an EOD drag coefficient of 2.5 would yield a molar consumption of $1+2.5$. One mole is consumed for the water molecule being oxidized, and 2.5 of the water molecules are being dragged through the ionomer. The EOD drag coefficient is temperature dependent only and modeled using an empirical correlation (11).

$\mathrm{n}_{\mathrm{H} 2 \mathrm{O}}=2.9 \exp \left[1029\left(\frac{1}{333}-\frac{1}{\mathrm{~T}}\right)\right]$

Methanol cross-over due to EOD is accounted for in a very similar manner, however it is based on the molar fraction of water (12) present in the anode catalyst layer.

$\mathrm{n}_{\mathrm{MeOH}}=\mathrm{n}_{\mathrm{H} 2 \mathrm{O}} \mathrm{X}_{\mathrm{MeOH}}$ 
Cathode Catalyst Layer: Similar to the anode catalyst layer, the cathode catalyst layer is modeled as a single domain of uniform porosity and permeability which the reduction of oxygen takes place. However all species transport is modeled using Stephan-Maxwell Diffusion (8) instead of Fick-type Diffusion. The electro-kinetics are modeled using the concentration dependent form of the Butler-Volmer equation (13).

$\mathrm{j}_{\mathrm{c}}=\mathrm{j}_{\mathrm{o}, \mathrm{c}}\left(\frac{\mathrm{C}_{\mathrm{O} 2}}{\mathrm{C}_{\mathrm{O}, \mathrm{ref}}}\right) \exp \left[\frac{\alpha_{\mathrm{c}} \mathrm{F} \eta_{\mathrm{c}}}{\mathrm{RT}}\right]$

The cathode exchange current density is also modeled using a temperature dependent empirical correlation (14).

$\mathrm{j}_{\mathrm{o}, \mathrm{c}}=0.04222 \exp \left[\frac{73200}{\mathrm{R}}\left(\frac{1}{353}-\frac{1}{\mathrm{~T}}\right)\right]$

In the cathode catalyst layer water is produced from the oxidation reduction reaction and also from electro-osmotic drag from hydrogen ion and methanol transport. A stoichiometric balance is made on the cathode with the anode catalyst layers using (11) and (12).

Methanol cross over is also accounted for in the cathode catalyst layer model. While methanol species is not accounted for in the catalyst layer itself, it is accounted for at the membrane and catalyst layer interface where it is assumed the oxidation reaction occurs infinitely fast and reduces the overall cell potential. The amount of potential cell reduction is dependent upon the total methanol flux to this interface which is characterized by a parasitic current density (15).

$\mathrm{j}_{\text {para }}=6 \mathrm{FN}_{\mathrm{MeOH}, \mathrm{mem}}$

This parasitic current density is subtracted from the total cathode current to determine the entire cell "bulk" current (16).

$\int j_{\text {cell }} d A=\int j_{a} d A=\int j_{c} d A-\int j_{p a r a} d A$

This yields a cell potential (17) which is dependent upon activation potential losses, ohmic losses, and indirectly methanol cross-over and concentration. 
$E=E_{e q}-\eta_{a}-\eta_{c}-\frac{1}{\sigma} \int j_{c e l l} d A$

\section{Membrane}

The membrane is modeled as a single domain using Fick-type diffusion to account for the diffusion of concentration driven transport of water and methanol. A second set of terms is added on to the Fickian diffusion equation to account for the flux due to electro-osmotic drag of water and methanol (18).

$\mathrm{N}_{\mathrm{j}}=-\mathrm{D}_{\mathrm{i}} \nabla \mathrm{c}_{\mathrm{i}}+\mathrm{n}_{\text {drag, } \mathrm{i}} \frac{\mathrm{j}}{\mathrm{F}}$

Ion transport is determined using Ohm's Law (19).

$\mathrm{j}_{1}=-\sigma_{1} \nabla \emptyset_{1}$

The conductivity of the membrane is dependent upon temperature and water content and is modeled using an empirical correlation (20).

$\sigma_{l}=(0.5139 \lambda-0.326) \exp \left[1268\left(\frac{1}{303}-\frac{1}{\mathrm{~T}}\right)\right]$

\section{Boundary Conditions:}

External boundary Conditions: The external boundary conditions on the anode inlet are derived from the testing parameters of the experimental data in order to help determine the validity of a model. The conditions are only valid for the "general solution" of the model in order to compare to experimental results.

The anode inlet is operated at 0 psig with a methanol mole fraction of $50 \%$. The cathode inlet has an inlet velocity $0.1 \mathrm{~m} / \mathrm{s}$ normal to the surface of the cathode GDL. Ideal air is used $(\mathrm{XO} 2=$ $21 \%)$ and is slightly humidified $(\mathrm{XH} 2 \mathrm{O}=0.023 \%)$. The cathode exhausts to a pressure of 0 psig.

Internal Boundary Conditions: For the internal boundary conditions the IFDL, anode GDL and catalyst layer all have continuity along their boundaries. It is identical for the cathode side of the cell as well. The membrane-anode interface share concentration $\left(C_{i, A D L}=C_{i, m}\right)$ and molar flux $\left(\mathrm{N}_{\mathrm{i}, \mathrm{ADL}}=\mathrm{N}_{\mathrm{i}, \mathrm{m}}\right)$. 
The cathode membrane and catalyst layer interface share the same concentration of water, however the concentration at the interface for methanol is zero (21).

$\mathrm{C}_{\mathrm{H} 2 \mathrm{O}, \mathrm{m}}=\mathrm{C}_{\mathrm{H} 2 \mathrm{O}, \mathrm{CDL}}, \mathrm{C}_{\mathrm{MeOH}, \mathrm{m}}=0$

It should be noted that while the membrane and catalyst layer share the same concentration values at their interface, this isn't necessarily an accurate representation of what is occurring in the physical cell. The model assumes water present in the membrane interface vaporizes at the cathode catalyst layer and doesn't remain in liquid form. Ideally this boundary should contain a more detailed model of the effects of water vaporization and its influence on water transport as this interface is critical for properly accounting for water transport across the membrane and catalyst layers.

Because methanol is consumed and oxidized only at the interface the consumption of methanol and oxygen along with the production of water are modeled using relationships (22) and (23).

$\mathrm{N}_{\mathrm{H} 2 \mathrm{O}, \mathrm{CDL}}=\mathrm{N}_{\mathrm{H} 2 \mathrm{O}, \mathrm{m}}+2 \cdot \mathrm{N}_{\mathrm{MeOH}, \mathrm{m}}$

$\mathrm{N}_{\mathrm{MeOH}, \mathrm{m}}=1.5 \cdot \mathrm{N}_{\mathrm{O} 2, \mathrm{CDL}}$

Results and Discussion

\section{General Solution}

To determine the validity of a model, a "general solution" for the model was used. The model was solved and ran under the same conditions in which experimental data was provided. Overall the general solution shows good agreement with experimental results with regards to the cell's polarization curve. The model was solved for the following operating conditions:

$$
\begin{gathered}
\mathrm{T}=70^{\circ} \mathrm{C} \\
\mathrm{X}_{\mathrm{MeOH}, \mathrm{in}}=0.50 \\
\mathrm{X}_{\mathrm{O}_{2}, \mathrm{in}}=0.21 \\
\mathrm{~V}_{\mathrm{in}}=0.1 \mathrm{~m} / \mathrm{s} \\
\mathrm{P}_{\mathrm{a}}=0 \mathrm{psig} \\
\mathrm{P}_{\mathrm{c}}=0 \mathrm{psig}
\end{gathered}
$$


Figure 35 below shows the molar fraction of methanol distributed through the anode side and the membrane of the fuel cell.

V_cell(6)=0.4 Surface: Molar Fraction of $\mathrm{MeOH}(1)$

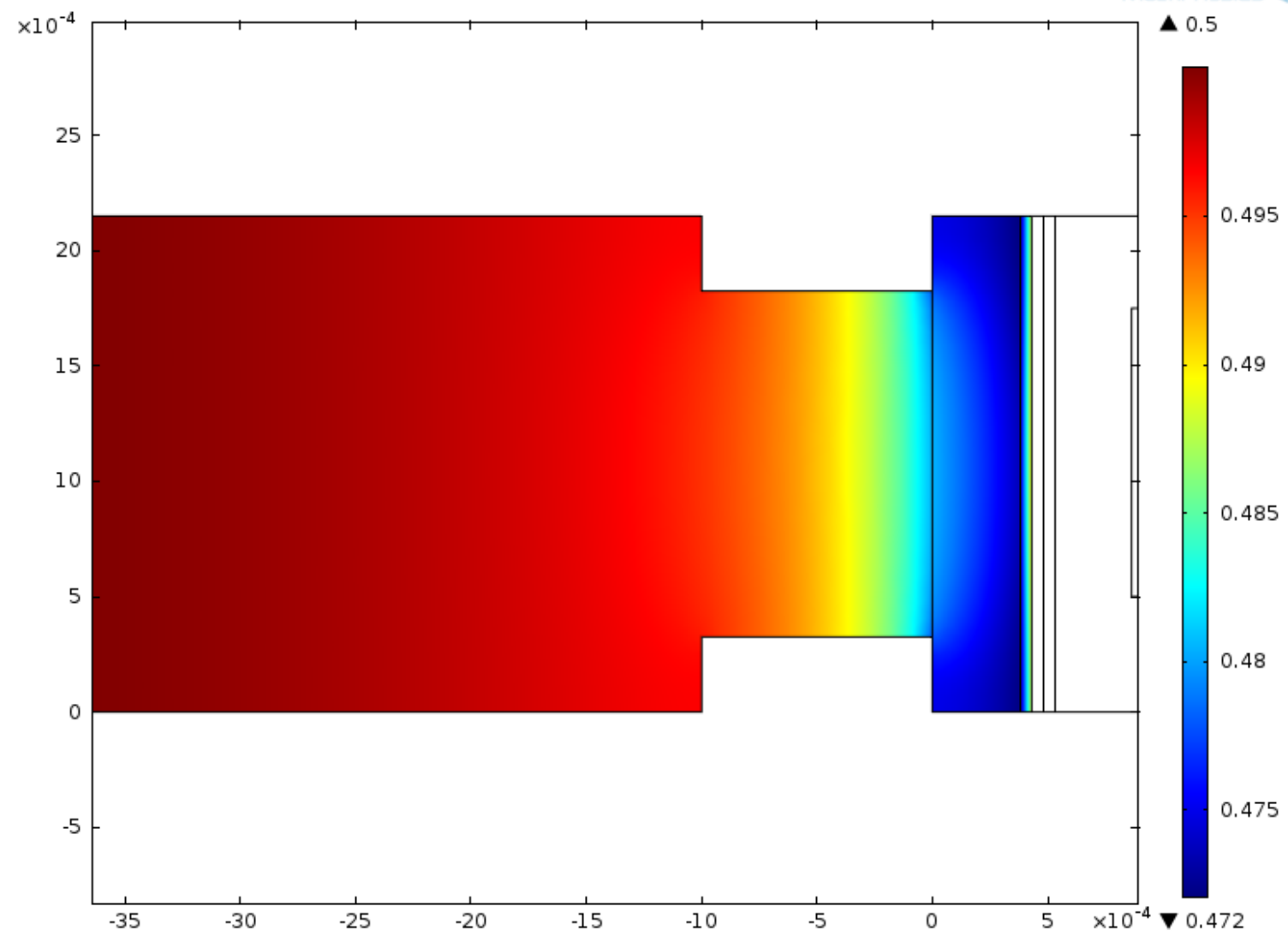

Figure 35: Mole Fraction of Methanol at 0.4V

As the surface plot shows there is a steady decrease in methanol molar fraction as the methanol travels to the anode. As will be shown, this is ultimately due to a greater flux of methanol due to diffusion through the membrane. Figure 36 shows the concentration distribution of water throughout the cell. 


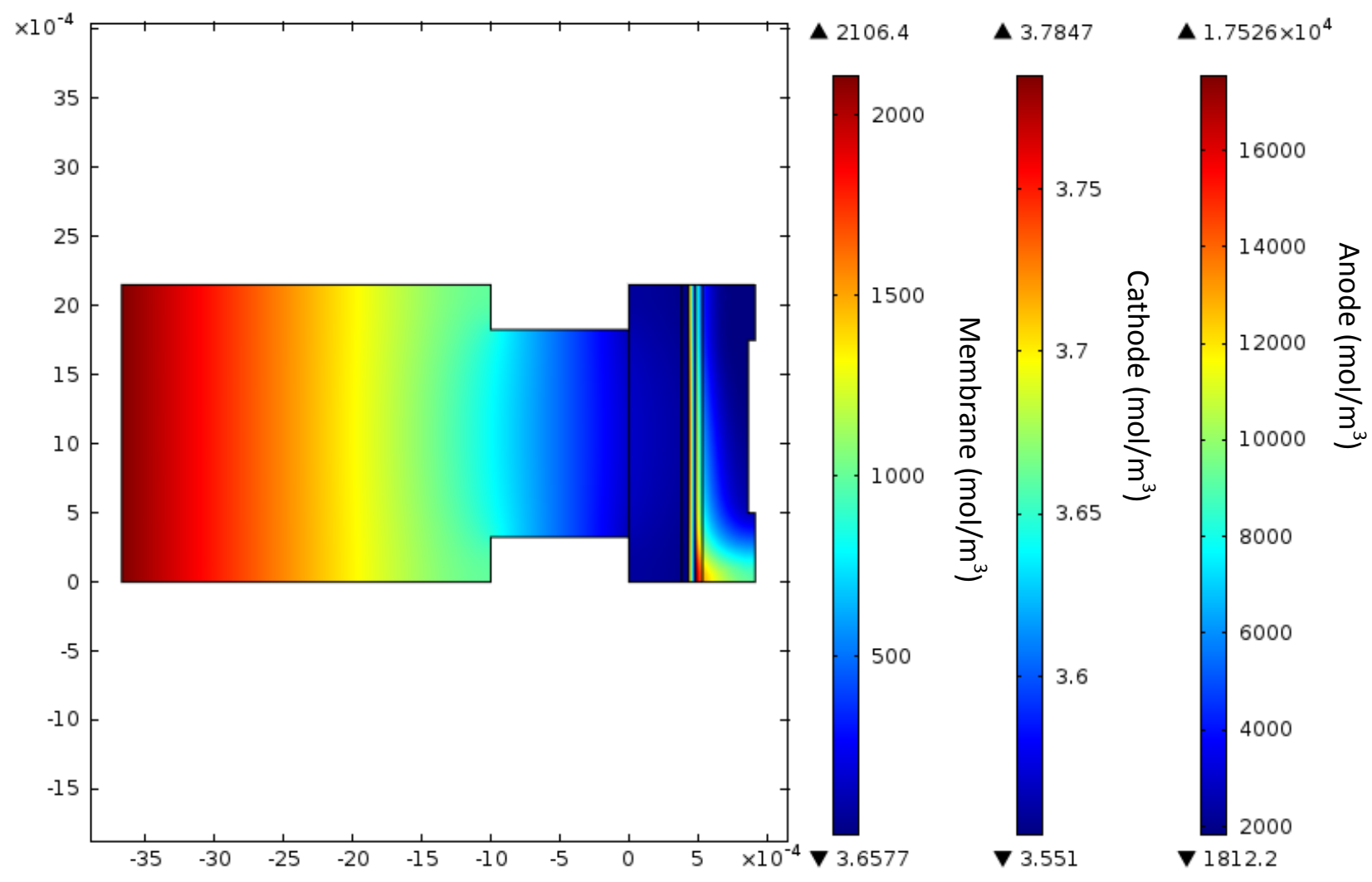

Figure 36: Molar concentration of water throughout cell at $0.4 \mathrm{~V}$

As the water flows from the anode inlet to the anode catalyst layer there is an obvious decrease in concentration as would be expected. However, what is not expected is the magnitude of the decrease. As the reactant species on the cathode are incompressible liquid it is expected that there would be little decrease in concentration. However, because Fick-type diffusion is used for species transport a large concentration gradient is required to create an adequate flux. Anode species transport could be better accounted for using Stephan-Maxwell diffusivity.

Figures 37 and 38 show the flux of water and methanol throughout the cell. As would be expected the flux of both species flows from the inlet to the right side of the cell. Both of these plots were generated for a cell potential of $0.9 \mathrm{~V}$. While this isn't open circuit voltage, it is an operating point with low current so the effects of water and methanol crossover can be observed. 

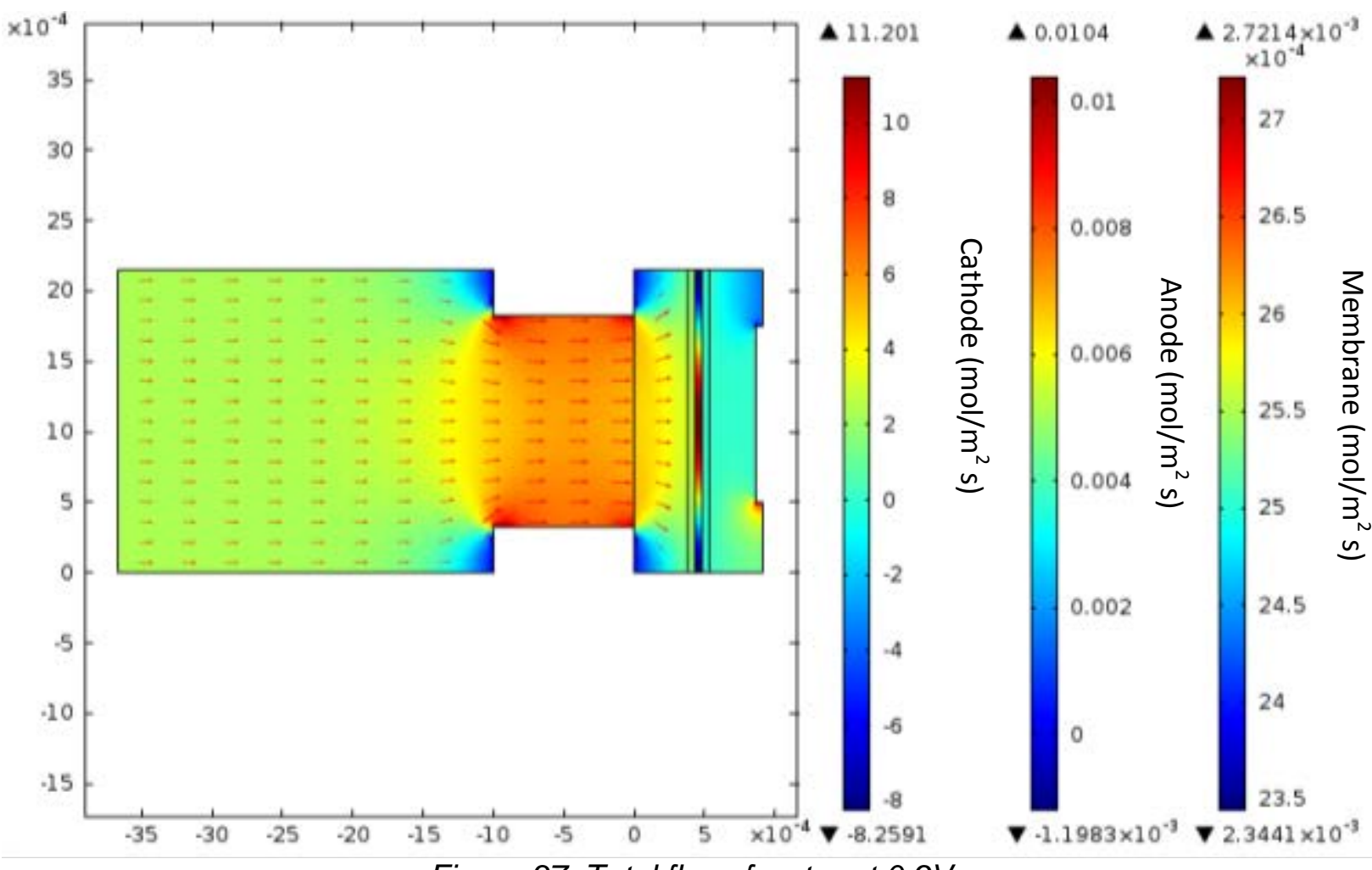

Figure 37: Total flux of water at 0.9V

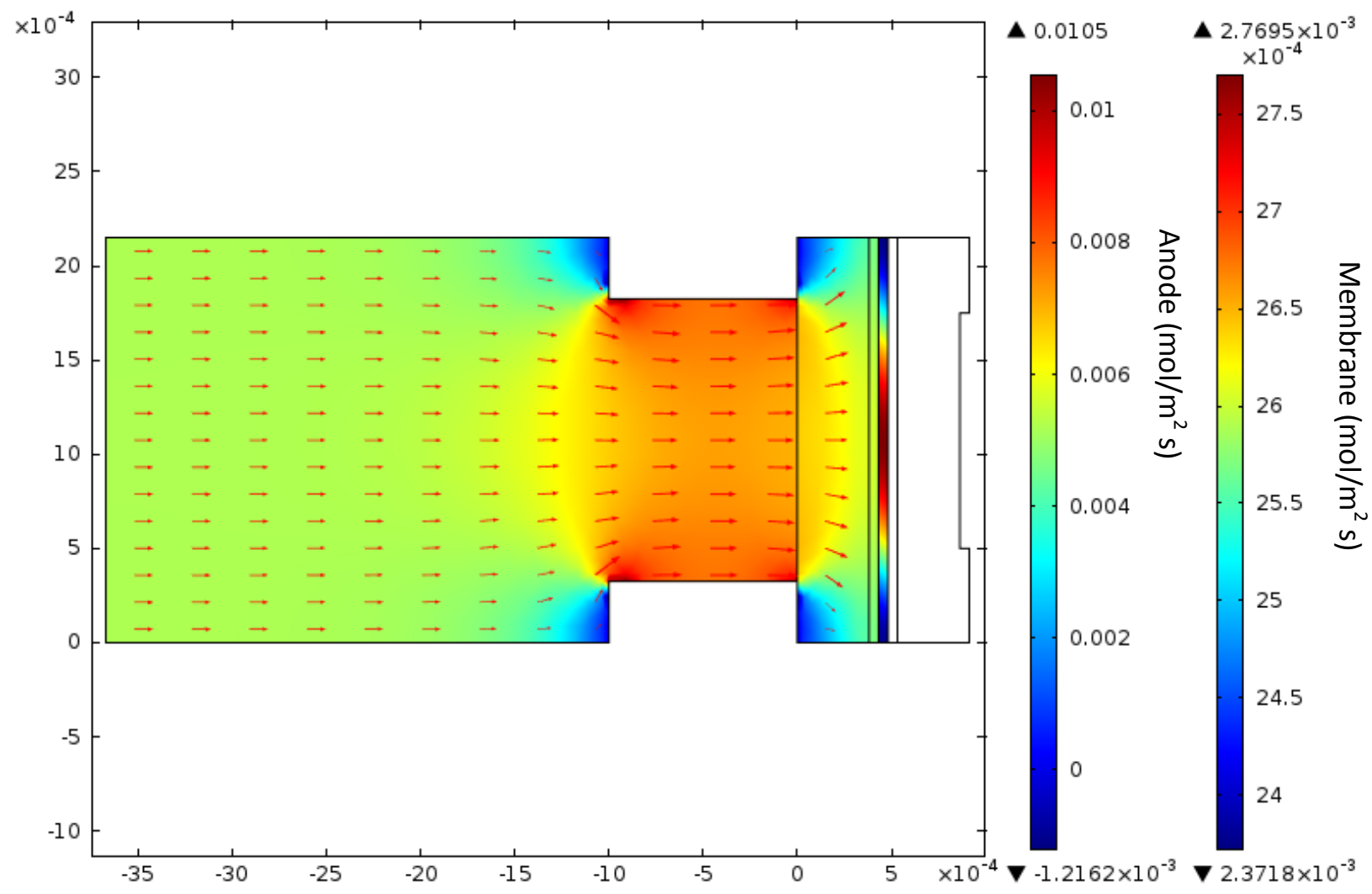

Figure 38: Total flux of methanol at $0.9 \mathrm{~V}$ 
From Figures 37 and 38 it can be seen that methanol has a larger total flux than water. This is due to two factors, the diffusivity coefficient for methanol is higher, and the concentration gradient for methanol is larger. The difference in concentration of is due to water being present in the cathode catalyst layer. While this water is in vapor form, it still creates a decrease in concentration gradient across the membrane.

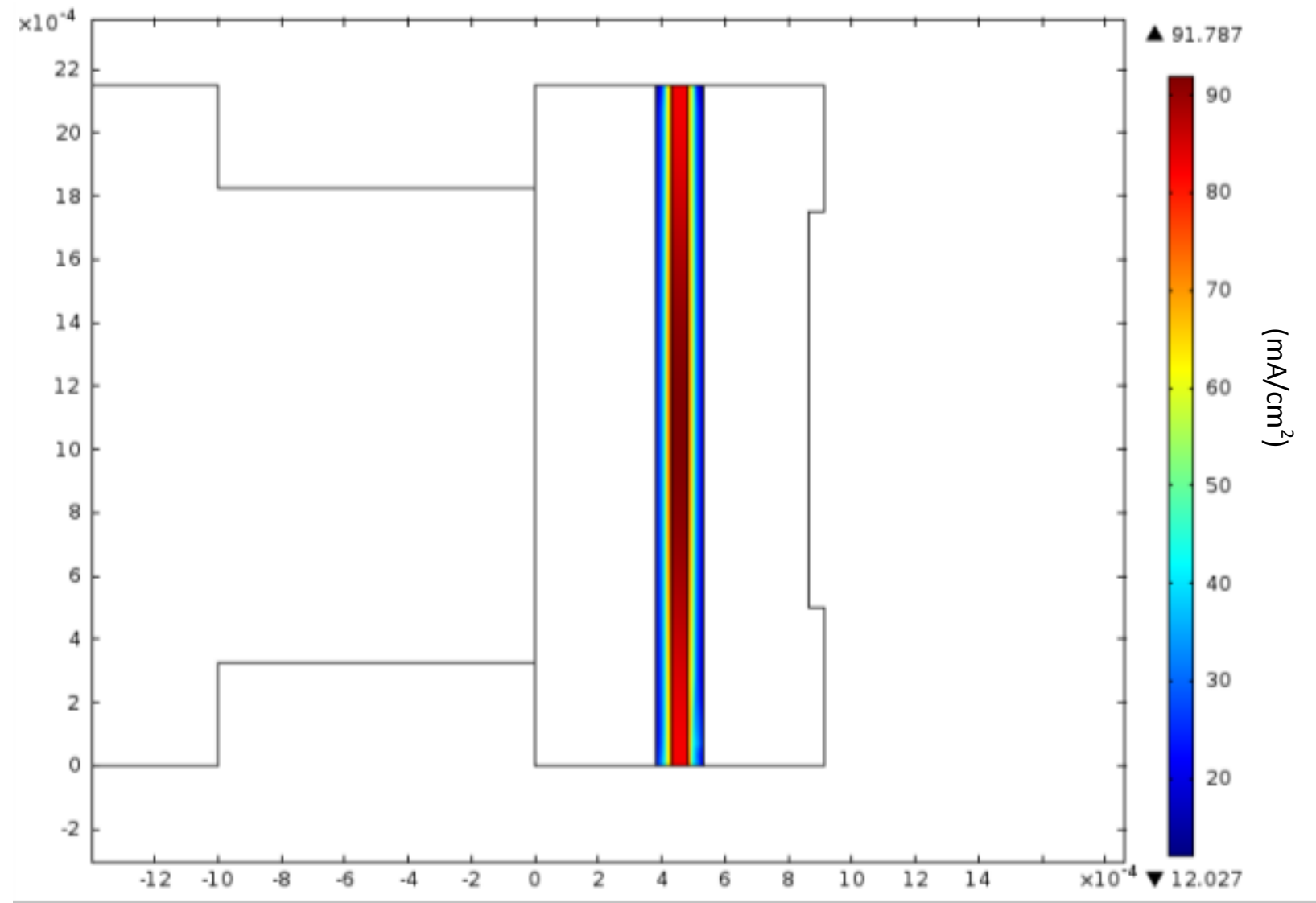

Figure 39: Ionic current distribution at $0.9 \mathrm{~V}$

Figure 39 shows the ionic current distribution in the membrane and catalyst layers. This is ultimately due to the placement of the flow channel rib (better conductivity) but is also influenced by the local concentrations of reactants.

While the in situ concentrations and flux of species of the experimental cell is nearly impossible to determine, the models validity can be estimated by comparing its polarization curve to that of the experimental fuel cell which is shown in Figure 40. 


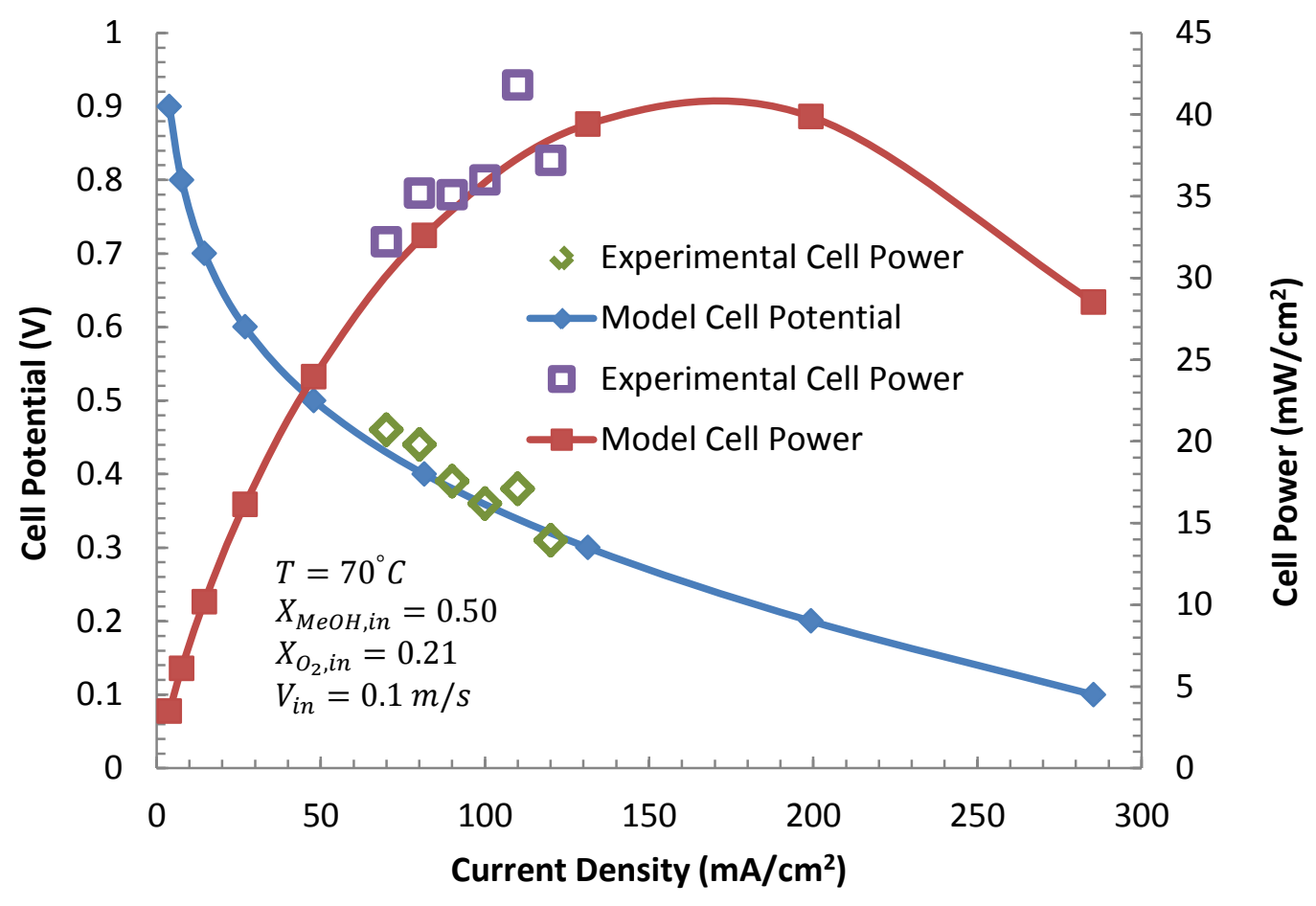

Figure 40: Polarization and power curves for DMFC model and experimental Data

As Figure 40 shows, there is relatively good agreement between the model and the actual experimental fuel cell results at this set of operating conditions. It should be noted that no "tuning" of the model has been performed. The results of the model are shown using values parameters form the literature and the experimental fuel cell itself. To obtain more confidence in the model the experimental fuel cell should be operated over a large band of current densities and other operating conditions.

\section{Case Studies}

To investigate the influence of different operating parameters on cell performance, the model was solved under several different operating conditions and parameters. These studies include:

- Four temperatures: $10,30,50,70^{\circ} \mathrm{C}$

- Three molar inlet fractions: 0.2, 0.5, 0.8

- Molar fraction of $\mathrm{H}_{2} \mathrm{O}$ on cathode: $0.0001,0.15,0.30$

- Tortuosity of cathode: 1.5, 3.0, 5.0 
The first study was to determine the influence of temperature. It was expected that at higher temperature cell performance would increase due to faster reaction kinetics and higher rates of reactant diffusivity. The cell polarization curves are shown in Figure 41.
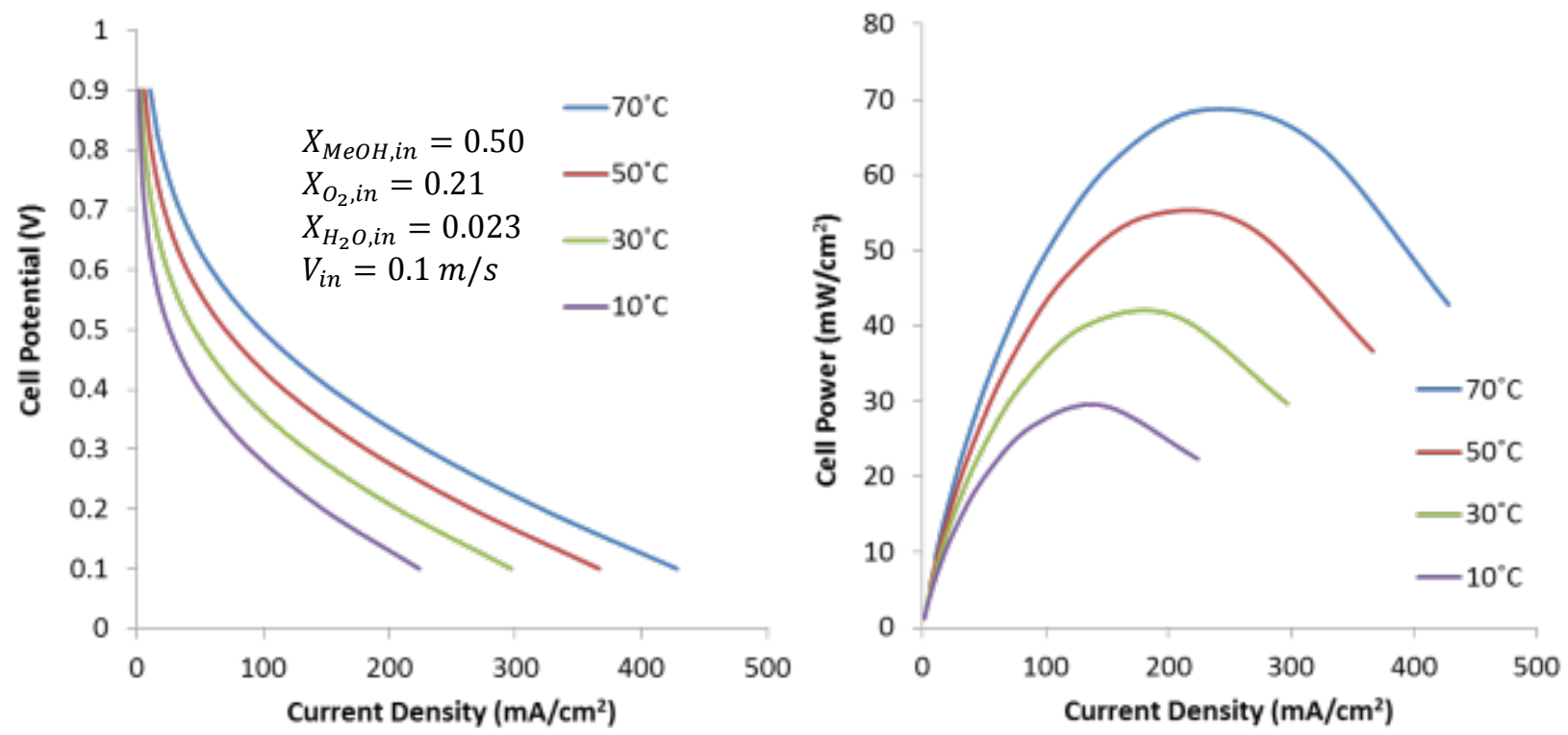

Figure 41: DMFC performance and different operating temperatures

As expected an increase in temperature causes an overall increase in performance of the cell. This is predominantly due to an increase in exchange current density which is highly temperature dependent.

The next study involved solving the model at different inlet molar fractions for methanol. Because a concentration dependent Butler-Volmer equation (9) \& (13) was used it was assumed that this would have a large influence on performance. 

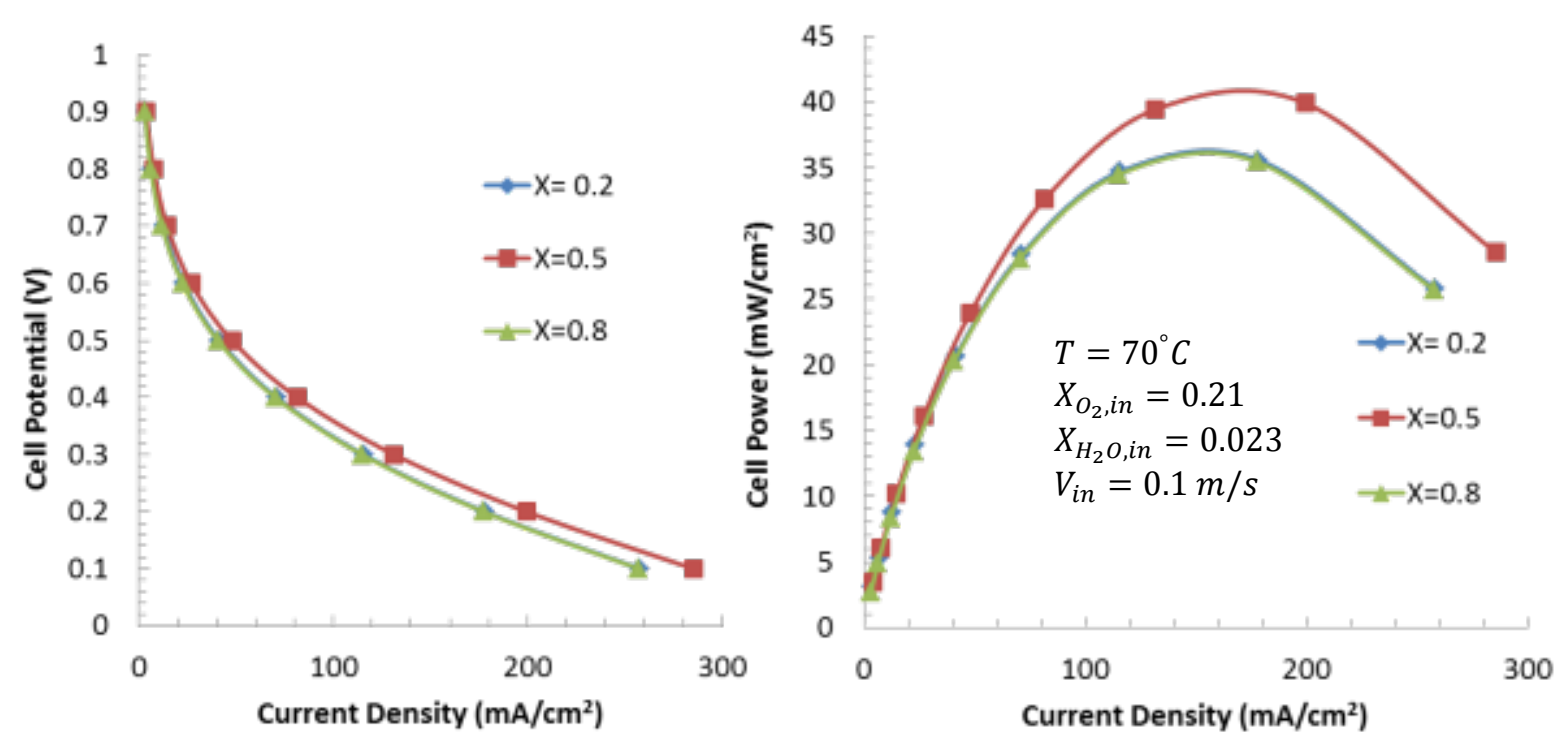

Figure 42: DMFC performance and varying methanol molar inlet flux

As Figure 42 shows, molar inlet fraction of methanol does give marginal performance changes and is not as large as would be expected. This is thought to be due to a rather low concentration of reactants at the anode catalyst layer due to the IFDL. Because the concentration at the anode is so low, large changes at the anode inlet would have a small affect. However, it should be noted that the optimal performance is achieved with a concentration of $50 \%$ (mole), which would be expected.

The next study involved operating the cell at different humidities at the cathode inlet. It was expected that higher humidities would provide much greater performance as it would enable less water transport through the membrane due to a smaller concentration gradient.
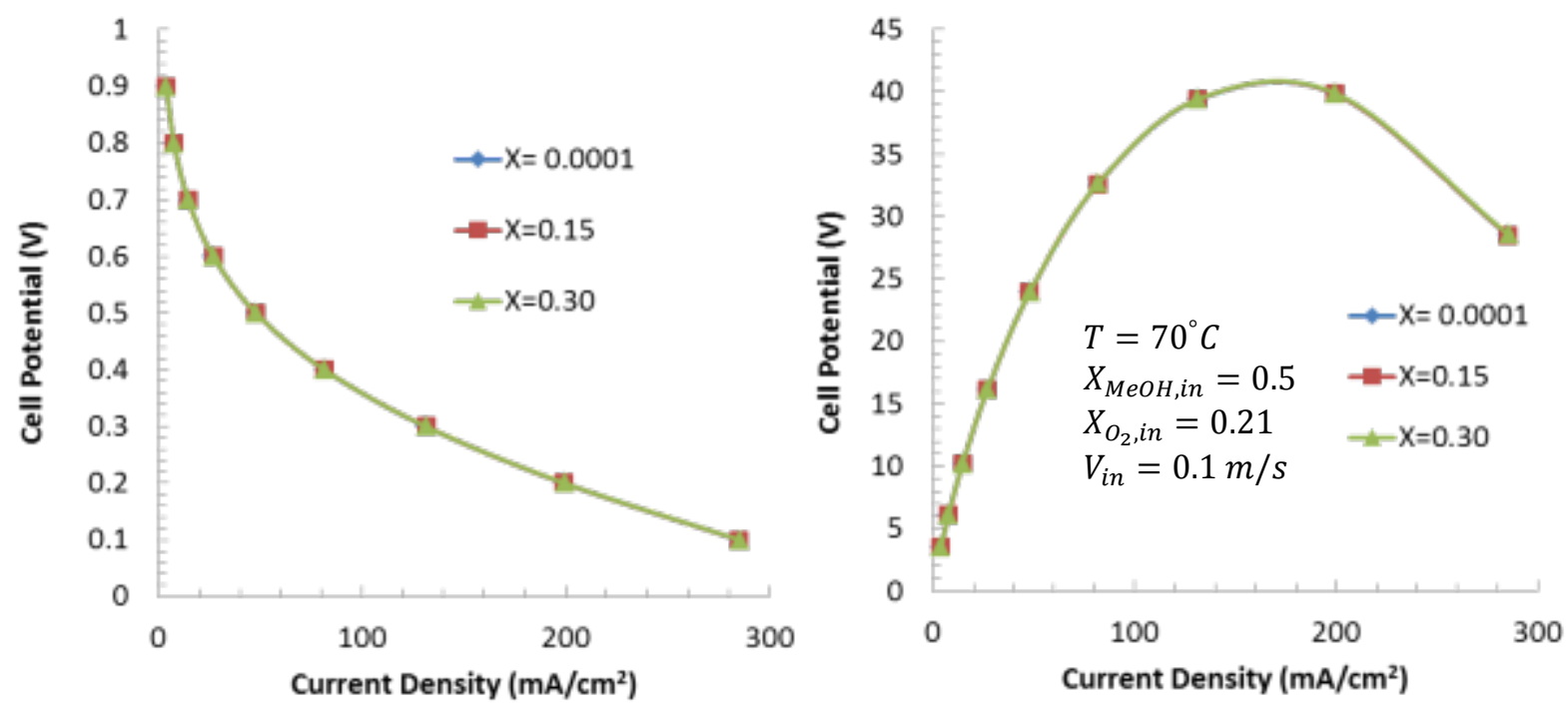

Figure 43: DMFC performance and varying cathode inlet humidity 
The results shown in Figure 43 suggest that cathode inlet humidity has little to no effect on the DMFC's performance. This is assumed to be due to the fact that the model is single phase on the cathode. Since the relative humidity change is relatively small with respect to concentration, this would have little influence on cell performance as it would yield only a slight change in total water flux from anode to cathode. However, if a two-phase model was used where liquid water was accounted for, changes humidity would have a significant influence on water evaporation and condensation and therefore cell performance.

The last study performed involved changing the tortuosity of cathode GDL and catalyst layer. The purpose was to modify the Bruggeman (4) correction in order to simulate flooding of the cell. Ideally, a decrease in porosity or the diffusion coefficient would be similar to liquid water obstructing reactant flow.
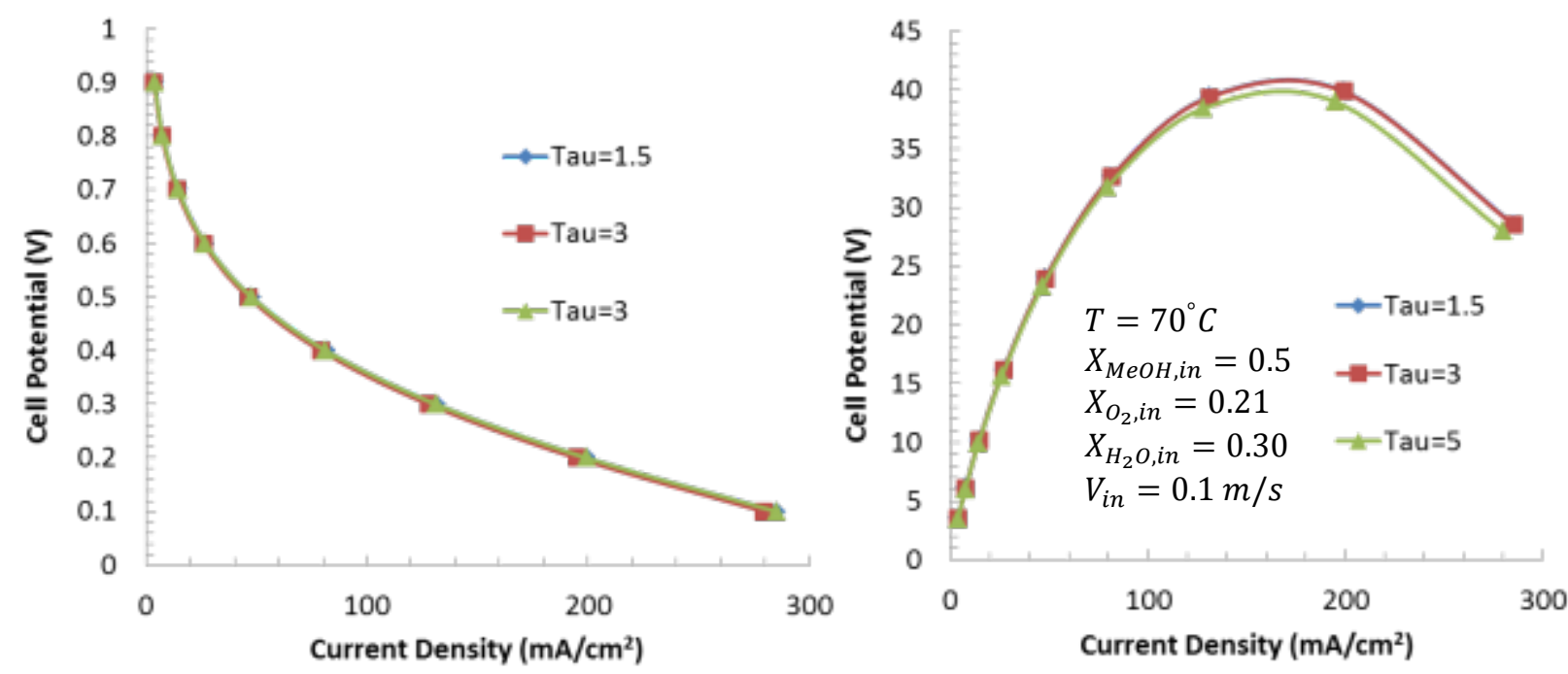

Figure 44: DMFC performance at varying tortuosites of the cathode catalyst and diffusion layer

As Figure 44 shows there was little to no change in performance when modifying tortuosity. This is thought to be due to the relatively low current density of DMFC's and their low requirement for reactant transport. Because the cell is operating at such low current densities, gas transport on the cathode gas diffusion layer (especially for forced convection) isn't necessarily critical. Another thing to note is that changes in diffusivity or tortuosity is uniform across the entire cathode. A more correct method of modeling water formation would be to use a two-phase model where effective porosity isn't uniform and more likely to occur in the catalyst layer. 


\section{Conclusions and Summary}

The current model is able to reasonably model cell performance under the provided operating conditions. However, the physics of species transport, especially with regards to liquid water on the cathode, is over simplified and needs to be further developed for the model to become a useful and practical cell model. More specifically, the two-phase physics need to be further developed and contain more detail about liquid transport in the cathode region. Furthermore, the model would also greatly benefit from more detailed electro-kinetics due to methanol crossover which is essential for accounting for cell losses and startup operation. More "tuning" of the model will be performed.

\section{Appendix:}

\section{Nomenclature and Values}

\begin{tabular}{|cll|}
\hline $\mathrm{H}_{\text {cell }}$ & $2.15[\mathrm{~mm}]$ & Height of Cell Model \\
$\mathrm{L}_{\text {ifdl }}$ & $3.67[\mathrm{~mm}]$ & IFDL Thickness \\
$\mathrm{L}_{\mathrm{gdlA}}$ & $0.381[\mathrm{~mm}]$ & Anode GDL Thickness \\
$\mathrm{L}_{\mathrm{clA}}$ & $0.050[\mathrm{~mm}]$ & Anode CL Thickness \\
$\mathrm{L}_{\mathrm{mem}}$ & $0.051[\mathrm{~mm}]$ & Membrane Thickness \\
$\mathrm{L}_{\mathrm{gdlC}}$ & $0.381[\mathrm{~mm}]$ & Cathode GDL Thickness \\
$\mathrm{L}_{\mathrm{clC}}$ & $0.050[\mathrm{~mm}]$ & Cathode CL Thickness \\
$\mathrm{MW}_{\mathrm{O} 2}$ & $32[\mathrm{~g} / \mathrm{mol}]$ & Molecular Weight of O2 \\
$\mathrm{MW}_{\mathrm{N} 2}$ & $28[\mathrm{~g} / \mathrm{mol}]$ & Molecular weight of N2 \\
$\mathrm{MW}_{\mathrm{H} 2 \mathrm{O}}$ & $18[\mathrm{~g} / \mathrm{mol}]$ & Molecular Weight of H2O \\
$\mathrm{MW}_{\mathrm{MeOH}}$ & $32[\mathrm{~g} / \mathrm{mol}]$ & Molecular Weight of MeOH \\
$\mathrm{MW}_{\mathrm{cO} 2}$ & $44[\mathrm{~g} / \mathrm{mol}]$ & Molecular Weight of CO2 \\
$\rho_{\mathrm{meoh}}$ & $0.8763[\mathrm{~g} / \mathrm{ml}]$ & Density of MeOH solution \\
$\mathrm{P}_{\mathrm{cell}}$ & $1[\mathrm{~atm}]$ & Reference Pressure \\
$\mathrm{V}_{\mathrm{O}}$ & 1.21 & Thermoneutral potential \\
$\alpha_{\mathrm{a}}$ & 0.239 & Anode transfer coefficient \\
$\alpha_{\mathrm{c}}$ & 0.875 & Cathode transfer coefficient \\
$\mathrm{A}_{\text {eff }}$ & $1[1 / \mathrm{m}]$ & Effective reaction surface area \\
$\mathrm{C}_{\mathrm{ref}, \mathrm{H} 2 \mathrm{O}}$ & $100\left[\mathrm{~mol} / \mathrm{m}^{\wedge} 3\right]$ & Reference concentration of water \\
$\mathrm{C}_{\mathrm{ref}, \mathrm{O} 2}$ & $0.136\left[\mathrm{~mol} / \mathrm{m}^{\wedge} 3\right]$ & Reference concentration of Oxygen \\
$\mathrm{C}_{\text {ref,MeOH }}$ & $100\left[\mathrm{~mol} / \mathrm{m}^{\wedge} 3\right]$ & Reference MeOH concentration \\
$\mathrm{a}_{\mathrm{H} 2 \mathrm{O}}$ & 1 & Water vapor activity \\
\hline
\end{tabular}




\begin{tabular}{|c|c|c|}
\hline $\mathrm{K}_{\mathrm{acl}}$ & $2.0 \mathrm{e}-15\left[\mathrm{~cm}^{\wedge} 2\right]$ & Permeability of anode CL \\
\hline $\mathrm{K}_{\mathrm{ccl}}$ & $2.0 \mathrm{e}-15\left[\mathrm{~cm}^{\wedge} 2\right]$ & Permability of cathode CL \\
\hline $\mathrm{K}_{\mathrm{adl}}$ & $5.0 \mathrm{e}-13\left[\mathrm{~m}^{\wedge} 2\right]$ & Permeability of anode GDL \\
\hline $\mathrm{K}_{\mathrm{cdl}}$ & $5.0 \mathrm{e}-13\left[\mathrm{~m}^{\wedge} 2\right]$ & Permeability of cathode GDL \\
\hline $\mathrm{K}_{\text {ifdl }}$ & $1.5 \mathrm{e}-10\left[\mathrm{~m}^{\wedge} 2\right]$ & Permeability of IFDL \\
\hline$\varepsilon_{\text {adl }}$ & 0.7 & Poroisty of Anode GDL \\
\hline$\varepsilon_{\mathrm{cdl}}$ & 0.7 & Porosity of cathode GDL \\
\hline$\varepsilon_{\mathrm{acl}}$ & 0.3 & Porosity of anode CL \\
\hline$\varepsilon_{\mathrm{ccl}}$ & 0.3 & Porosity of cathode CL \\
\hline$\varepsilon_{\text {ifdl }}$ & 0.363 & Porosity of IFDL \\
\hline$\varepsilon_{\mathrm{m}}$ & 0.3 & Porosity of Nafion Membrane \\
\hline$\sigma_{g d l}$ & $500[\mathrm{~S} / \mathrm{m}]$ & GDL Electrical Conductivity \\
\hline$\sigma_{\mathrm{cl}}$ & $500[\mathrm{~S} / \mathrm{m}]$ & Catalyst layer conductivity \\
\hline$\sigma_{\text {ifdl }}$ & $198[\mathrm{~S} / \mathrm{m}]$ & Electrical conductivity of IFDL \\
\hline $\mathrm{T}$ & 1.5 & Tortuosity of GDL and CL \\
\hline $\mathrm{T}_{\mathrm{m}}$ & 1.8 & Tortuosity of Nafion membrane \\
\hline $\mathrm{O}_{\text {nafion }}$ & 0.4 & Ionomer fraction of $\mathrm{CL}$ \\
\hline $\mathrm{O}_{\text {carbon }}$ & 0.4 & Electrode fraction of $\mathrm{CL}$ \\
\hline$\mu_{\mathrm{a}}$ & $1.19 \mathrm{e}-5\left[\mathrm{~Pa}{ }^{*} \mathrm{~s}\right]$ & Anode viscosity \\
\hline$\mu_{c}$ & $2.46 \mathrm{e}-5\left[\mathrm{~Pa} \mathrm{~A}^{\star} \mathrm{s}\right]$ & Cathode viscosity \\
\hline
\end{tabular}

$\underline{\text { Variables }}$

\begin{tabular}{|ccl}
\hline$\lambda_{303 \mathrm{~K}}$ & $0.043+17.81 a_{\mathrm{H} 2 \mathrm{O}}-39.85 a^{2}+36.0 a^{3}$ & Equilibrium water content at 303K \\
$\lambda_{353 \mathrm{~K}}$ & $0.3+10.8 a_{\mathrm{H} 2 \mathrm{O}}-16 a^{2}+14.1 a^{3}$ & Equilibrium water content at 353K \\
$\lambda^{*}$ & $\lambda_{303 \mathrm{~K}}+\frac{\lambda_{353 \mathrm{~K}}-\lambda_{303 K}}{50}(T-303)$ & Interpolated water content \\
$D_{\mathrm{MeOH}, \mathrm{H} 2 \mathrm{O}}$ & $6.69^{-9}$ & Diffusivity of methanol in water \\
$D_{\mathrm{MeOH}, \mathrm{Naf}}$ & $4.9 e^{-10} \exp \left[2436\left(\frac{1}{333}-\frac{1}{T}\right)\right]$ & Diffusivity of methanol in Nafion \\
$D_{\mathrm{H} 2 \mathrm{O}, \mathrm{Naf}}$ & $2.6 e^{-6} \exp \left[2060\left(\frac{1}{303}-\frac{1}{T}\right)\right]$ & Diffusivity of water in Nafion \\
\hline
\end{tabular}




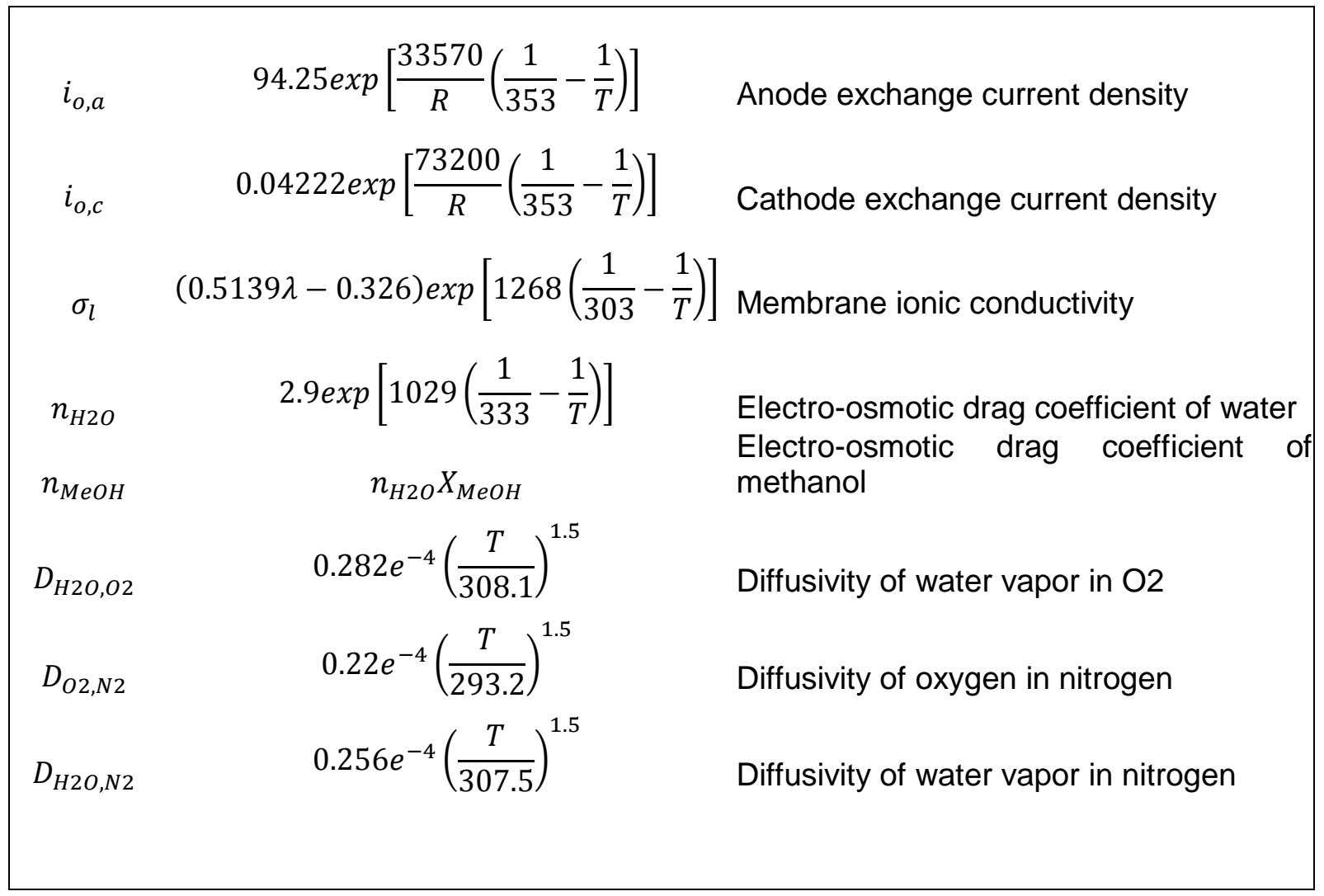




\section{Milestone\#6: Manufacture gas diffusion layers with user defined levels} of hydrophobicity.

The passive or semi-passive direct methanol fuel cell relies on internal water management to mitigate need for an outside water supply. The DMFC produces three times more water at the cathode than is necessary in the anodic reaction. In the classic (or legacy) DMFC technology, the water from the cathode is being exhausted in the surroundings and the anode operates with extremely dilute methanol solutions. This scenario renders the classic technology commercially unviable due to the extremely low energy density of the system. At least one third of the water produced at the cathode must be returned to anode either through external means or internally through back diffusion from the cathode to the anode.

A schematic of the pathway for water formation and transport in the DMFC cathode is shown in figure 45.

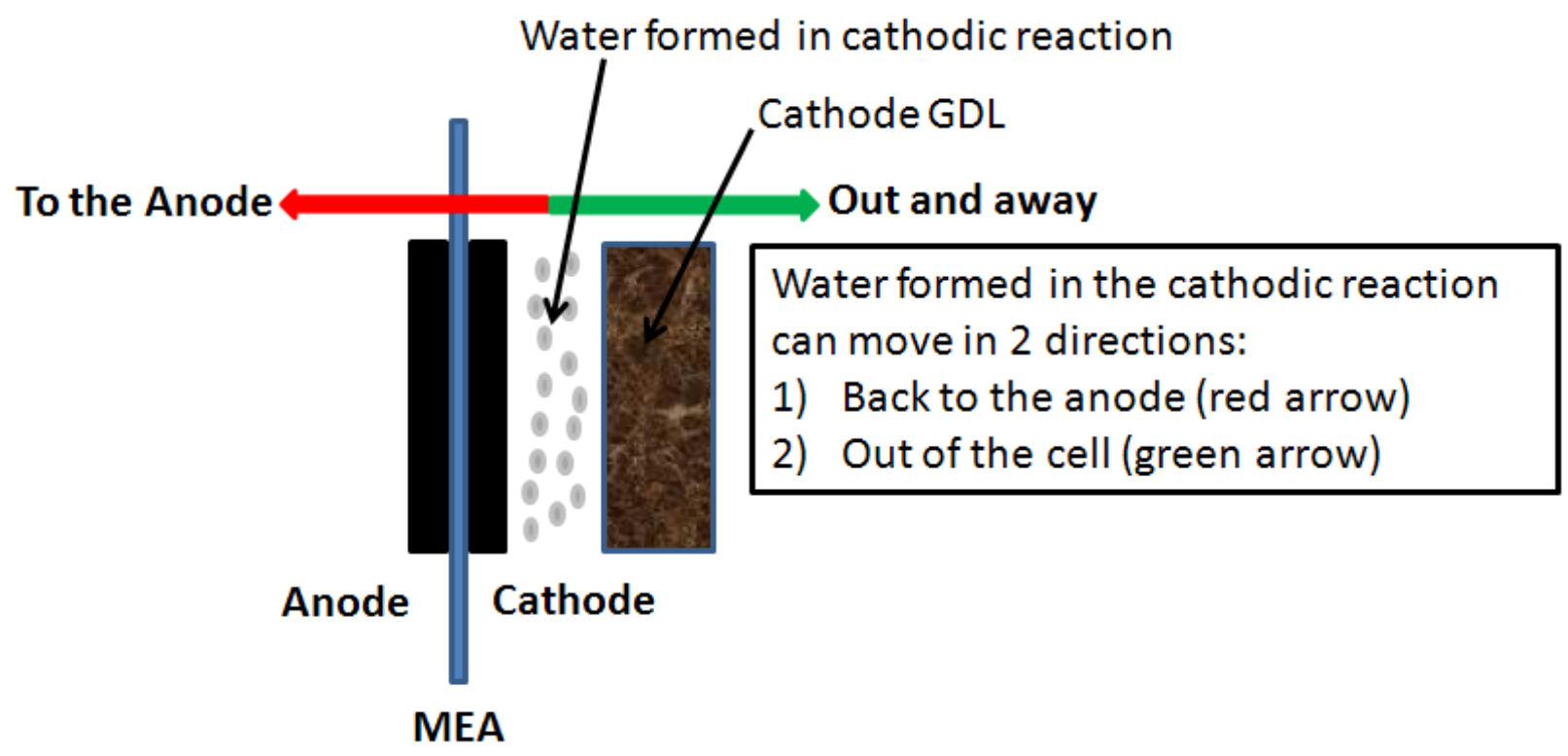

Figure 45. Direction of water flow in the IFDL DMFC

The rate at which 1 mole of water is produced at the cathode of a DMFC operating at, for example, $100 \mathrm{~mA} / \mathrm{cm}^{2}$ is given by the following formula: 


$$
\mathrm{R}_{\text {water }}=\frac{0.1 \cdot \frac{\mathrm{A}}{\mathrm{cm}^{2}} \cdot 18 \cdot 18 \cdot \frac{\mathrm{gm}}{\mathrm{mole}}}{2 \cdot 96485 \frac{\mathrm{A} \cdot \mathrm{sec}}{\mathrm{mole}}}=168 \cdot \frac{\mu \mathrm{gm}}{\mathrm{cm}^{2} \cdot \mathrm{sec}}
$$

At least $1 / 3$ of the water generated needs to be returned from the cathode to the anode to aid in the methanol oxidation reaction. The flux of water that needs to be forced back into the anode is:

$$
\frac{168}{3}=56 \cdot \frac{\mu \mathrm{gm}}{\mathrm{cm}^{2} \cdot \mathrm{sec}}
$$

It has been determined that the hydraulic permeability of water through Nafion 112 is roughly $17.3 \mu \mathrm{gm} /\left(\mathrm{cm}^{2}{ }^{*} \mathrm{sec}^{*} \mathrm{~atm}\right)$. Therefore, in order to be able to force $56 \mu \mathrm{gm} /\left(\mathrm{cm}^{2}\right.$ *sec) of water back to the anode the following pressure drop will be required:

$$
\frac{56 \cdot \frac{\mu g m}{\mathrm{~cm}^{2} \cdot \mathrm{sec}}}{17.3 \cdot \frac{\mu \mathrm{gm}}{\mathrm{cm}^{2} \cdot \mathrm{sec} \cdot \mathrm{atm}}}=3.237 \mathrm{~atm}
$$

It is known that the capillary pressure that a hydrophobic pore exerts on liquid water trying to go through it is given by:

$$
\Delta p=\frac{2 \cdot \sigma}{d}
$$

where:

$\sigma=$ surface tension of water in contact with air $=71.18 \mathrm{dynes} / \mathrm{cm}$ at $30^{\circ} \mathrm{C}$ $\mathrm{d}=$ pore diameter of the hydrophobic pores of the microporous layer

Therefore the maximum diameter that the pores of the cathode GDL microporous layer should have in order to force the appropriate amount of water back through Nafion 112 to the anode and not allow it to escape through the cathode GDL is: 


$$
\mathrm{d} \leq \frac{2 \cdot \sigma}{\Delta \mathrm{p}}=\frac{2 \cdot 71.18 \frac{\text { dy nes }}{\mathrm{cm}}}{3.237 \mathrm{tam}}=0.434 \mu \mathrm{m}
$$

All the passive direct liquid fuel cells rely on in-situ dilution versus bulky external recirculation. The hydrophobic diffusion layer must also allow for oxygen diffusion into the cathode where the oxygen reduction reaction takes place. The optimal recipe for microporous layer hydrophobicity - pore diameter - porosity must be determined and this can only be accomplished with the ability to have a supplier with the ability and desire to customize the product upon customer specification. The US Company, ETEK, used to fabricate and sell specialty diffusion layers based on customer specification. Upon acquisition by BASF, ETEK discontinued sales of the low temperature diffusion layers. As the ETEK ELAT stock dwindled, it became obvious that NuVant had to build its own ELAT production facility to produce the required diffusion material. The operation of the IFDL DMFC could not proceed without the proper diffusion material on the cathode side. Below is the description of the production facility built at NuVant. Figure 46 shows the moving Doctor-Blade glass table required for casting the micro-porous layer onto carbon cloth or carbon paper. After the casting, the strip remains on the glass for drying (Figure 47). Prior to the deposition of the microporous layer, the carbon cloth or paper is hydrophobized by soaking it in a Teflon solution. As described earlier the IFDL program at DOE required very high loading of Teflon in the casting ink in order to reject the water and force it back to the anode. The production facility enabled NuVant to prepare new IFDL fuel cells and stacks, to acquire data for the modeling effort.

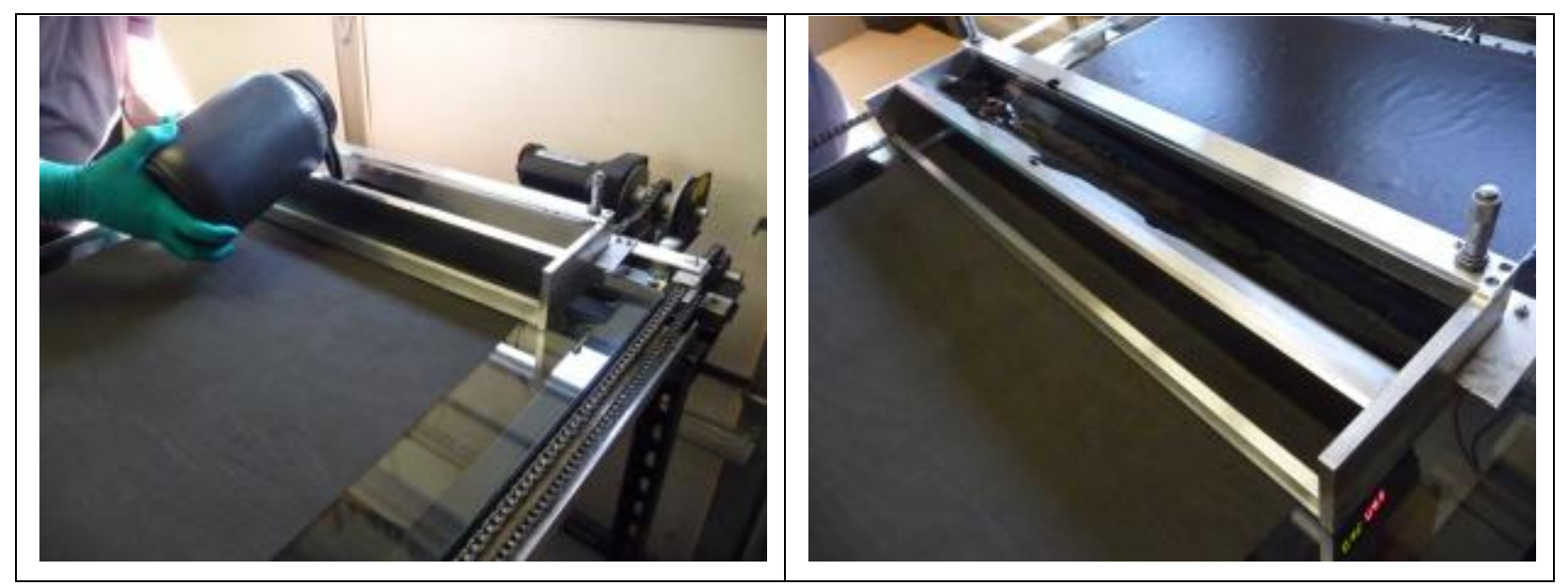

Figure 46. Diffusion layer casting table with Doctor-Blade driving mechanism. 


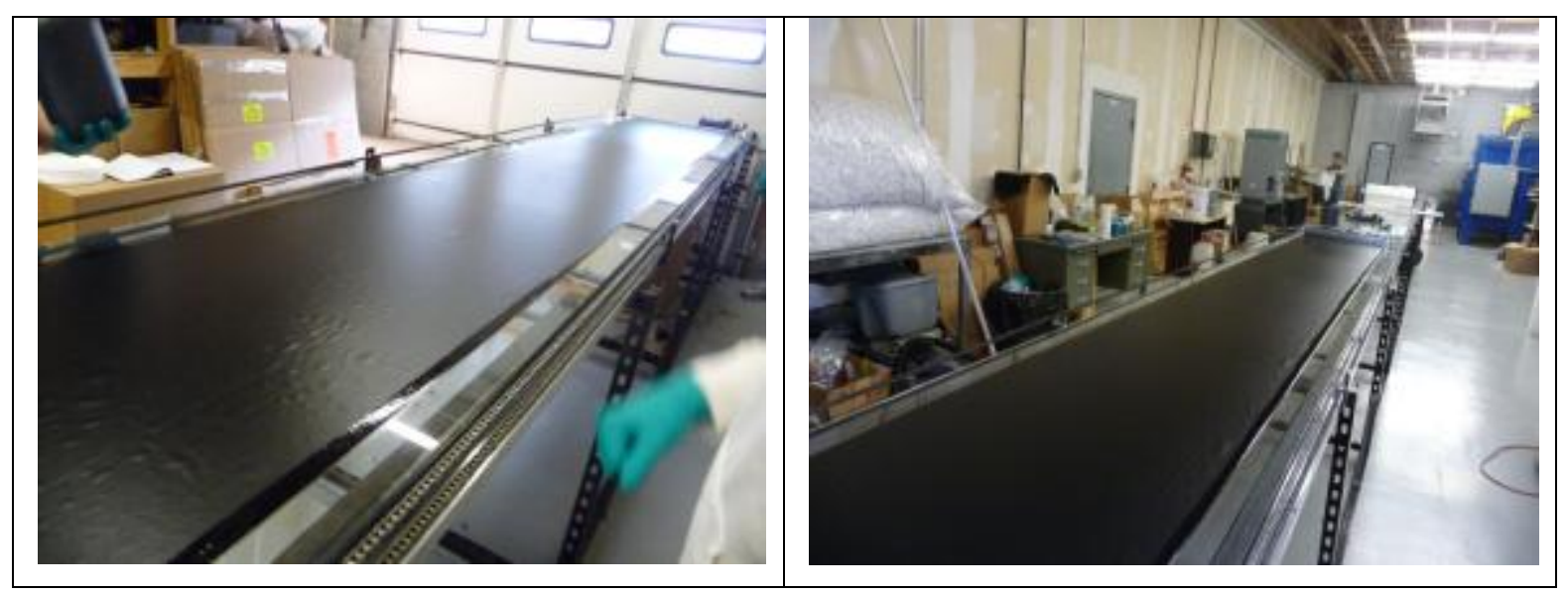

Figure 47. Diffusion layer Drying Table with high temperature baking oven in the background (right hand side photo background).

In order to be successful in the optimization of hydrophobic GDLs a number of tools needed to be constructed that enabled the measurement of certain parameters of utmost importance for the characterization of the diffusion layers.

We have purchased, designed and implemented equipment dedicated to measurement of thickness uniformity, electrical conductivity and gas/liquid permeability.

The thickness uniformity is being assessed with a high precision micrometer as shown in figure 48.

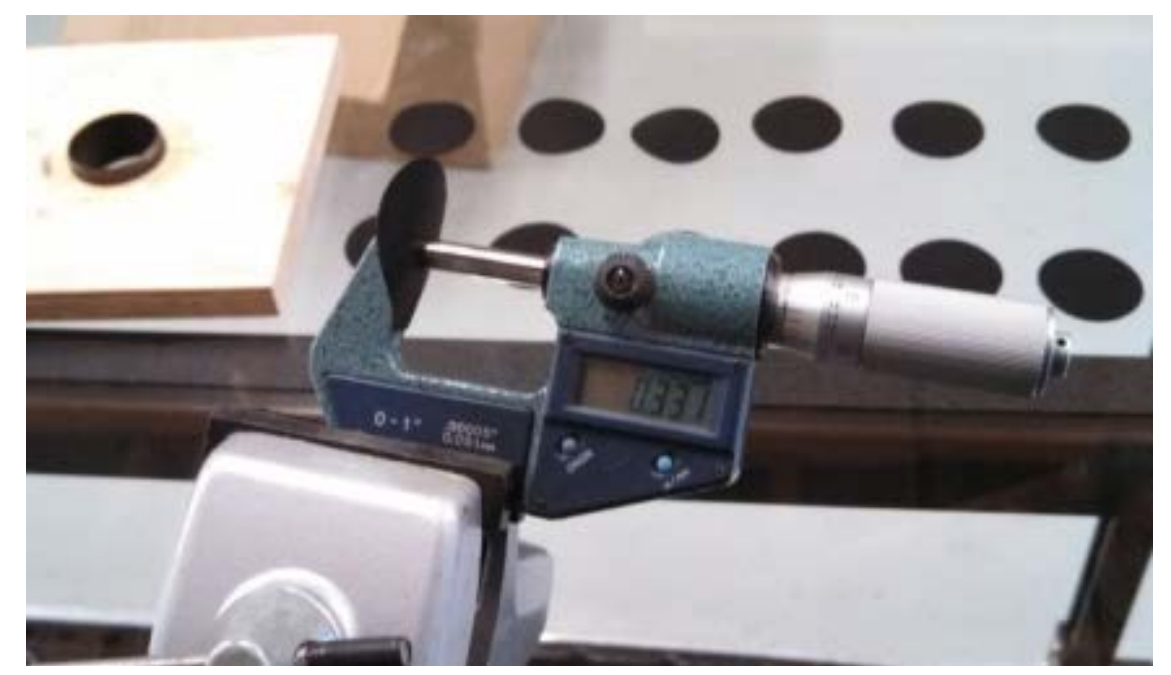

Figure 48. The thickness of NuVant's GDL circular coupons is measured with a high precision digital micrometer 
Circular coupons are cut off from various areas of the diffusion electrode and their thickness is measured thus assessing the uniformity of the electrode. An example of such measurement is show in the following table.

Table 1. The standard deviation of NuVant's GDL thickness is extremely low

\begin{tabular}{|r|r|r|r|r|}
\hline & \multicolumn{3}{|c|}{ Thickness (mm) } & \\
\hline & Sample 1 (across width) & Sample 2 (across width) & Sample 2 (across length) & Plain Carbon Cloth \\
\hline & 0.350 & 0.335 & 0.331 & 0.278 \\
& 0.368 & 0.323 & 0.328 & 0.289 \\
& 0.357 & 0.320 & 0.320 & 0.279 \\
& 0.352 & 0.316 & 0.314 & 0.267 \\
& 0.356 & 0.308 & 0.320 & 0.273 \\
& 5 & 0.313 & 0.308 & 0.268 \\
& 5 & 0.306 & 0.318 & $\mathrm{~N} / \mathrm{A}$ \\
& 6 & 0.358 & 0.310 & $\mathrm{~N} / \mathrm{A}$ \\
& 7 & 0.347 & 0.306 & $\mathrm{~N} / \mathrm{A}$ \\
\hline & 0.338 & 0.315 & 0.326 & 0.276 \\
\hline Average & $\mathrm{N} / \mathrm{A}$ & 0.306 & 0.321 & 0.267 \\
\hline Min & 0.353 & 0.335 & 0.308 & 0.289 \\
\hline Max & 0.338 & 0.029 & 0.331 & 0.022 \\
\hline Amplitude & 0.368 & 0.010 & 0.023 & 0.008 \\
\hline St. Deviation & 0.030 & & & 0.007 \\
\hline
\end{tabular}

The electrical resistivity of the electrodes is also of utmost importance since the flow of electrons involved in the electrochemical reaction(s) passes through them. The resistivity is measured via an AC impedance mili-ohmeter connected to two gold plated blocks sandwiching the circular coupons used in the thickness measurement. The setup in shown in figure 49:

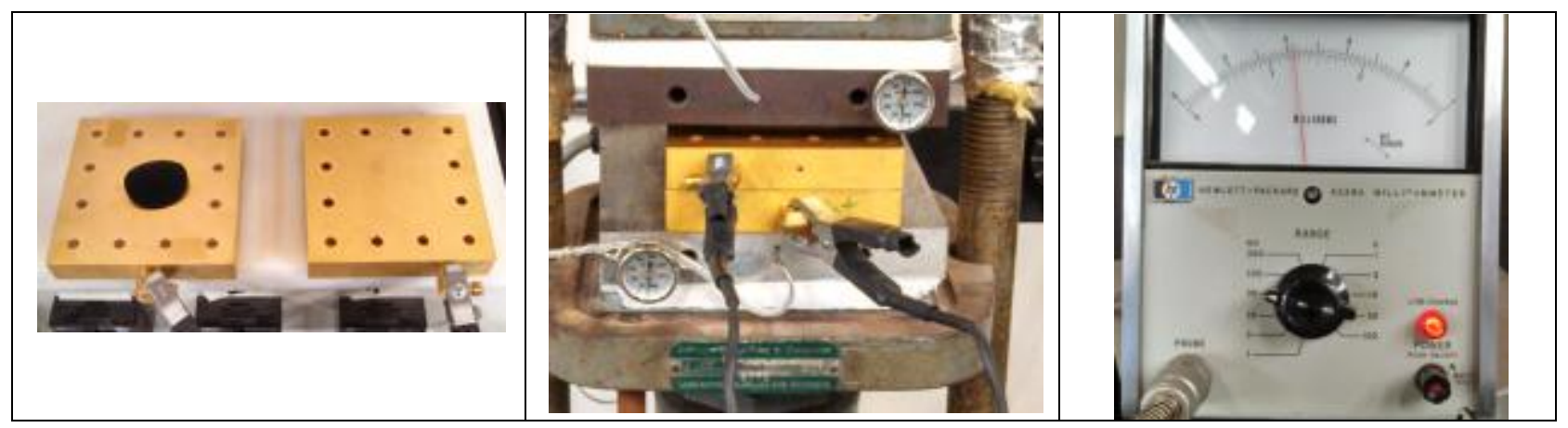

Figure 49. Apparatus for measuring the electrical resistance of NuVant's GDLs

The circular coupons were placed between the 2 gold plated plates shown at most left in figure 49. The assembly was inserted into the press shown in the middle of figure 49 in order to determine how the electrical resistivity changed with the compression, which is in accordance with what happens in the real fuel cell. An example of the result is shown in figure 50. 


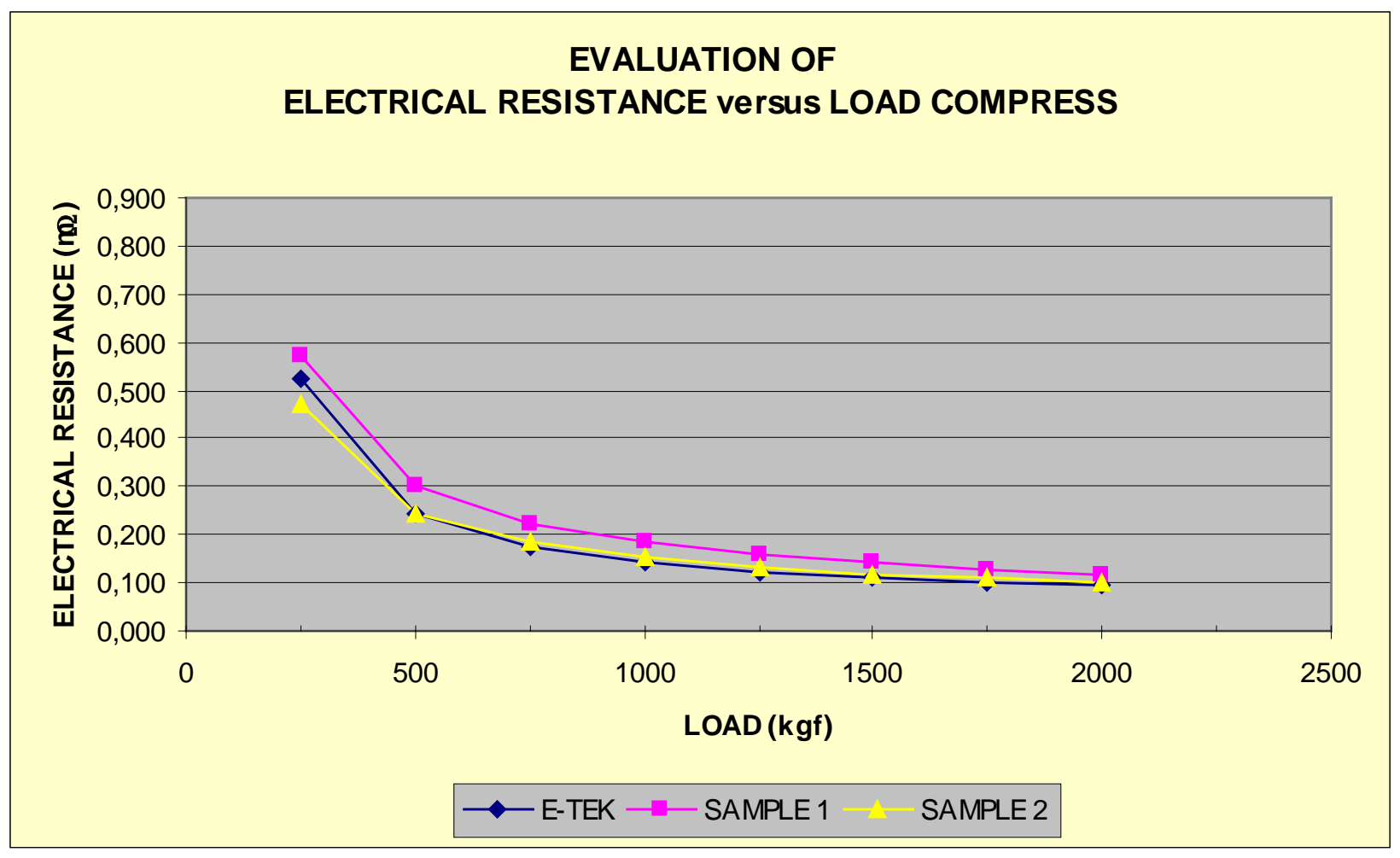

Figure 50. Change in electrical resistance with compression load

It can be seen from figure 50 that the electrical resistance of NuVant's GDL (sample 2) is virtually identical with what E-TEK used to offer which was considered state-of-the-art.

This type of ELAT diffusion electrodes are almost always used in flow reactors such as fuel cells, flow batteries and PEM electrolyzers so the permeability of the reactants and reaction products to and from the diffusion electrodes is also very important. This parameter is usually assessed by measuring the pressure drop that occurs when a known flowrate of the fluid of interest is passed through the diffusion electrode. The apparatus that measure the said pressure drop is shown in figure 51: 


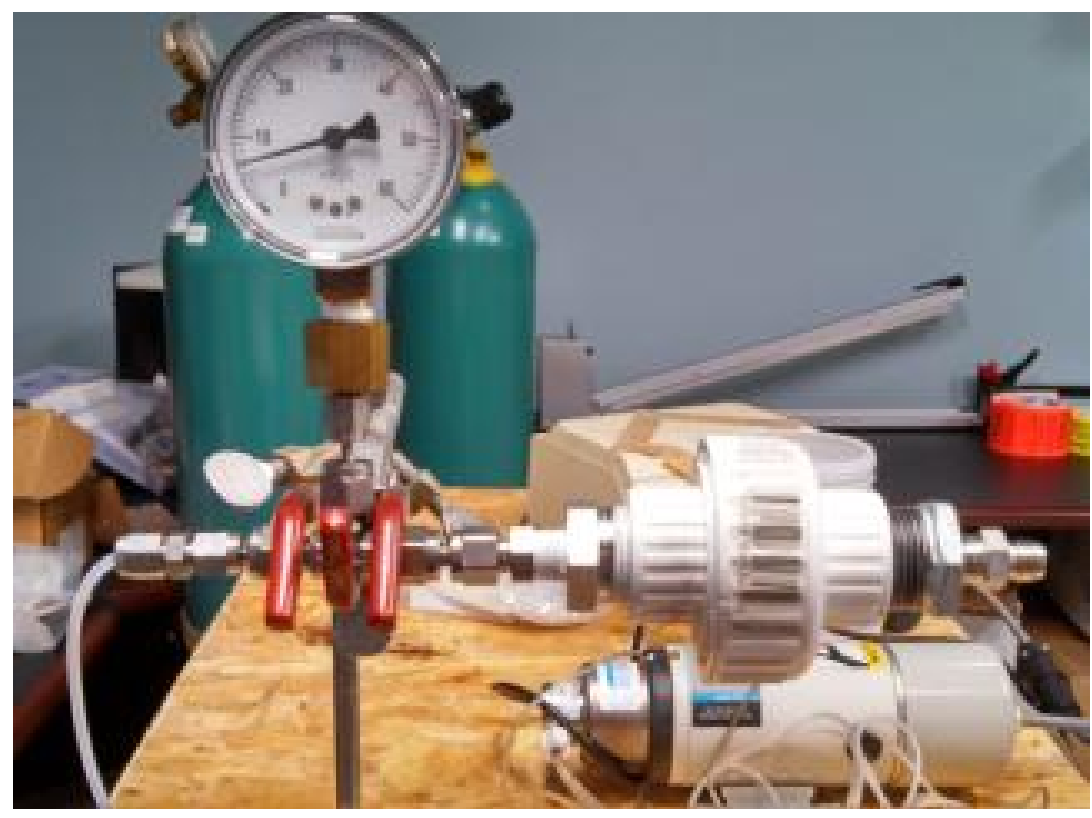

Figure 51. Gurley type device to determine the permeability of GDLs

Typical results obtained with the Gurley device are shown in Table 2.

Table 2. Pressure drop measurements using the Gurley-type device

PRESSURE DROP (mbar)

\begin{tabular}{|l|c|c|}
\hline Air Flow 5.0 SLPM; backpressure 0.7 bar & ESTAB 01 & ESTAB 02 \\
\hline E-TEK & & \\
\hline ELAT® - MEA 1 & 94 & 94 \\
\hline ELAT®- MEA 2 & 44 & 50 \\
\hline ELAT® - MEA 3 & 50 & 52 \\
\hline ELAT® - MEA 4 & 44 & 44 \\
\hline
\end{tabular}

A higher pressure drop through a gas diffusion layer induces parasitic energy losses due to the additional work required by compressors. The NuVant Product is $100 \%$ better than the E-TEK material.

An image of the hydrophobic material (water drop beads up) shown in comparison to the hydrophilic substrate that was used as a backbone is shown in figure 52. 


\section{Hydrophilic}

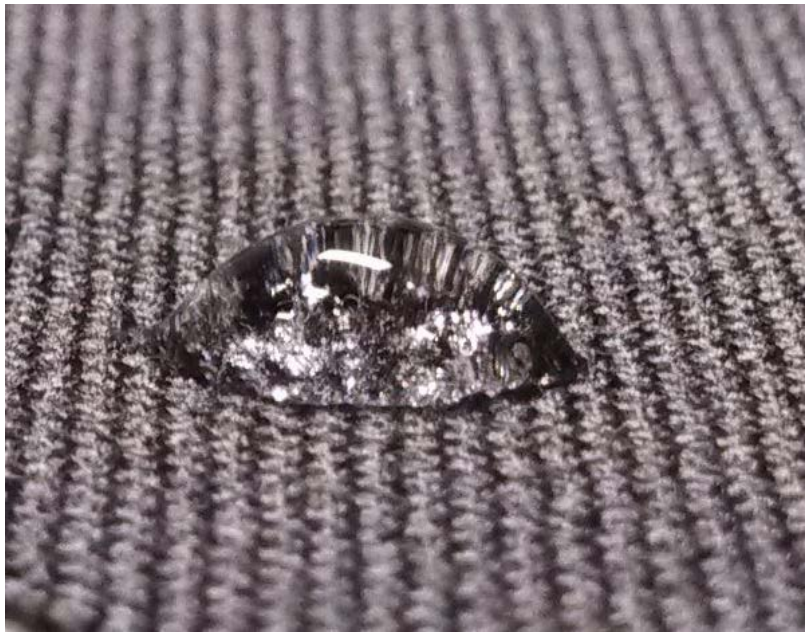

\section{Hydrophobic}

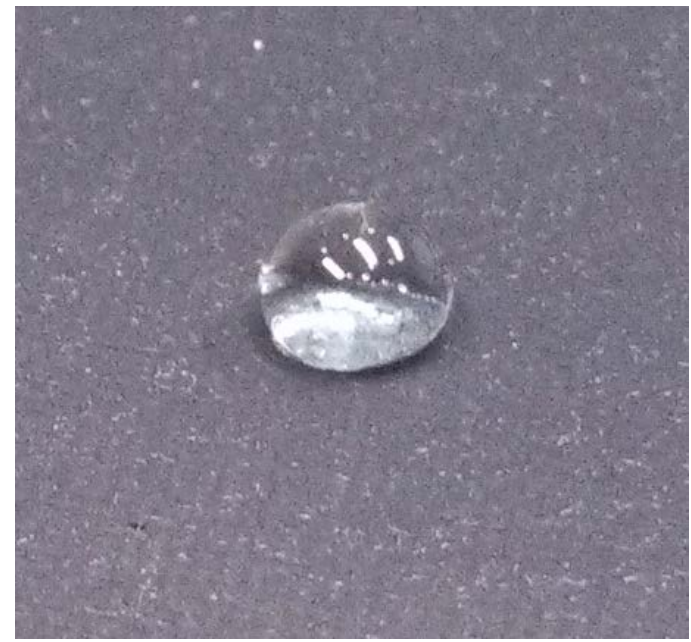

Figure 52. Hydrophobic diffusion layer (right) shown in comparison with the hydrophilic diffusion layer (left)

We can now fabricate diffusion layers with various layers of hydrophobicity to properly block water from escaping the fuel cell cathode and be returned to the anode to create the in situ dilution of methanol. 


\section{Milestone\#7: Optimize the cathode flow field design in order to accomplish uniform flow throughout the entire electrode area.}

It had become obvious during the operation of the IFDL DMFC that the cathode flowfield was not operating optimally, with plugs of water shooting out at fairly regular intervals of time. This is most often than not a sign of conduit flooding. NuVant has decided to build a tool that would enable visualization of flow with the purpose of designing a better flow field.

\section{Modular Flow Field Test Fixture}

In order analyze the fluid flow distribution over a flow field, a modular flow field test fixture was designed and fabricated. The test fixture will be used to simulate fluid flow through a flow field. Using this fixture, flow patterns through different flow field designs can be easily observed. The fixture is designed to the same scale as NuVant Systems' current flow fields.

\section{Initial Design Concept}

There are several initial design requirements for the modular flow field test fixture. First, it must allow for clear, easy viewing of fluid flows within it. Secondly, it must allow for any flow field type to be tested in it. Third, it must allow for flexibility in inlet and outlet configurations. Finally, it must be completely watertight, so as to avoid compromising testing. Several of these requirements were met in the initial fixture design. A 3D rendering of the initial design can be found in Figure 53.

The test fixture will be used to make observational analyses of fluid flow through flow fields. The fuel mixture will be pumped into the inlet by a syringe pump at a rate of $8 \mathrm{~mL} / \mathrm{min}$. The syringe is connected to the barbed fitting at the inlet by a 0.25 " inside diameter tube. The fuel will then flow through the pegboard. For testing, fuel will be substituted with a red-colored water solution to allow for observation of flow patterns in the test fixture. The Pegboard Assembly was designed as a means by which to test multiple methods of inlet flow manipulation. It consists of a series of pins arranged in the path of the fluid flow. This is to manipulate the flow in an attempt to achieve uniform flows into a flow field. is designed so that several peg configurations can be made in an attempt to manipulate the fuel flow to achieve the desired uniform flow across the flow field. The pegs are 3/32" in diameter and made of stainless steel. The fuel then passes over the flow field being tested and exits through the outlet into a reservoir. The entire fixture is covered by a sheet of .25" thick plexi-glass, which is lined with a closed-cell neoprene gasket. A 3D model rendering produced in Pro-Engineer of the initial test fixture design is shown in Figure 53 below. 
The housing was designed as a solid piece of black delrin to ensure that there is no risk of any fuel leaking out of the fixture. The fittings at the inlet and outlet are made from nylon. Plastic fittings were chosen because the team did not want to run the risk of ruining the tapped holes in the delrin with steel fittings. The Pegboard Assembly is constructed from two delrin plates. The top plate is made of white delrin to allow for a clear backdrop to view how the dyed solution interacts with the flowfield. Figure 20 on the next page shows an exploded view of the pegboard:

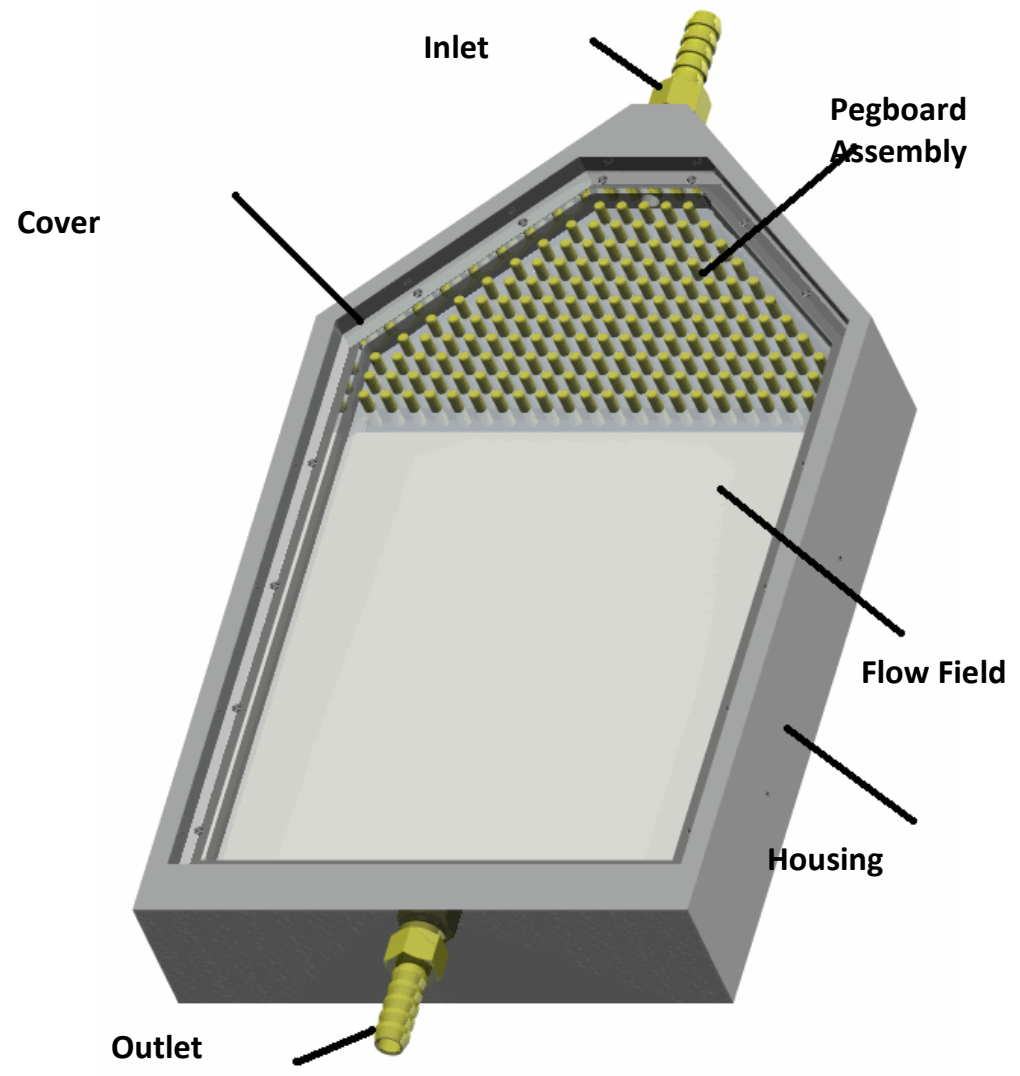

Figure 53: Modular flow field test fixture: Initial design 


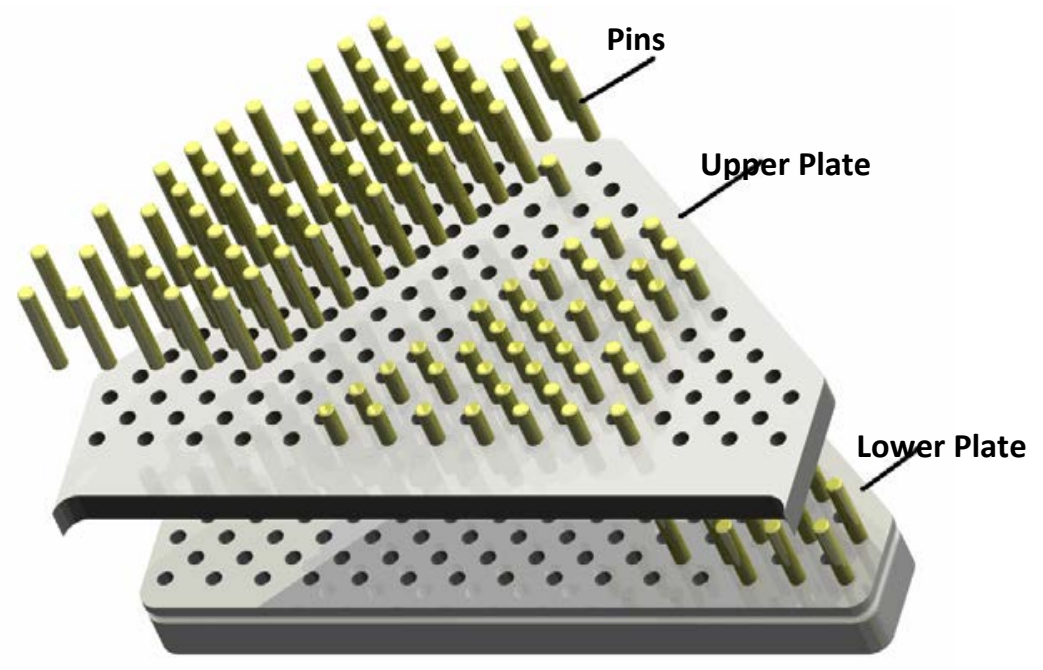

Figure 54: Exploded view of pegboard assembly.

The Pegboard Assembly is a two piece, removable subassembly. It was designed to be removable so that additional peg configurations could be used to allow for testing flexibility. It was designed as two separate plates to allow for easier removal of pins. Figure 54 shows an exploded view of the Pegboard Assembly with a series of pegs installed. When installing into the fixture, the pegs are first inserted in the lower plate. The holes in the lower plate create an interference fit with the pins, ensuring a tight fit and seal. The pins are installed in whatever arrangement the designer wishes to test. Two pin lengths were chosen, both with a diameter of 3/32". One set of pegs are 5/8" tall so that they extend up to inside surface of the cover. The second set is $3 / 8$ " tall. These pins are meant to serve as a hole filler, meaning that the top surface of the pin will be flush with the top surface of the upper plate once it is installed, providing a smooth surface for the fuel to flow over. The holes cannot be left blank because that could disturb the fluid flow and negatively affect experimental data. The Upper Plate has the same hole pattern machined into it, with a hole diameter .002" larger than the Lower Plate. This is to allow still for an sealing interference fit, while also allowing for this piece to be removed while the pins remain anchored in the Lower Plate. This is to allow for easy removal of the pins that are mounted flush with the surface of the Upper Plate when the component is fully assembled. There is an O-ring installed on the Lower Plate, to ensure a watertight seal. The Pegboard Assembly shown in Figure 54 has a staggered, diamond-shaped hole pattern. A second pegboard was designed with the holes in a parallel, square pattern shown in Figure 55 to allow for more pin configuration options. 


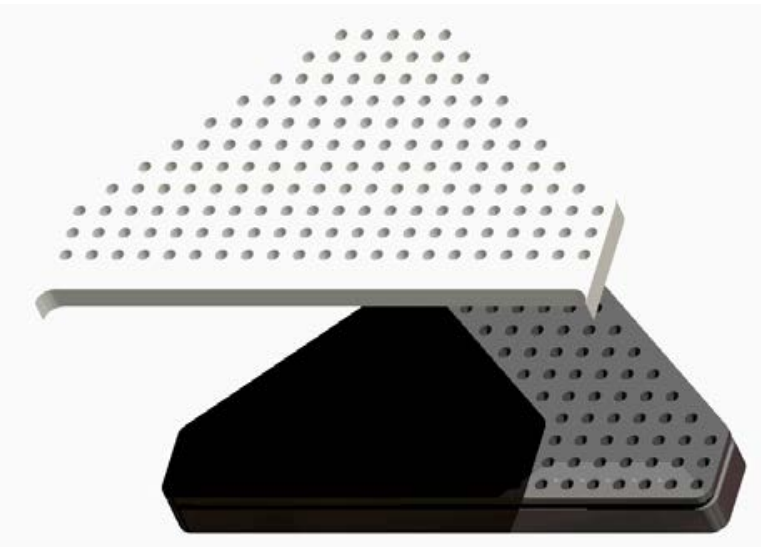

Figure 55: Square pattern pegboard assembly.

Final Design

Following a design review of the initial test fixture design, several problems were discovered.

First of all, a one-piece housing did not allow for the maximum flexibility of the fixture. It only had provisions for a single shape of an inlet pin field and no pin field at the outlet. Also, the one piece design made for a more difficult machining process than necessary. This problem was solved by incorporating a three-piece design, with interchangeable components. Assembled and exploded views of the assembly can be found in Figures 56 and 57, respectively. 


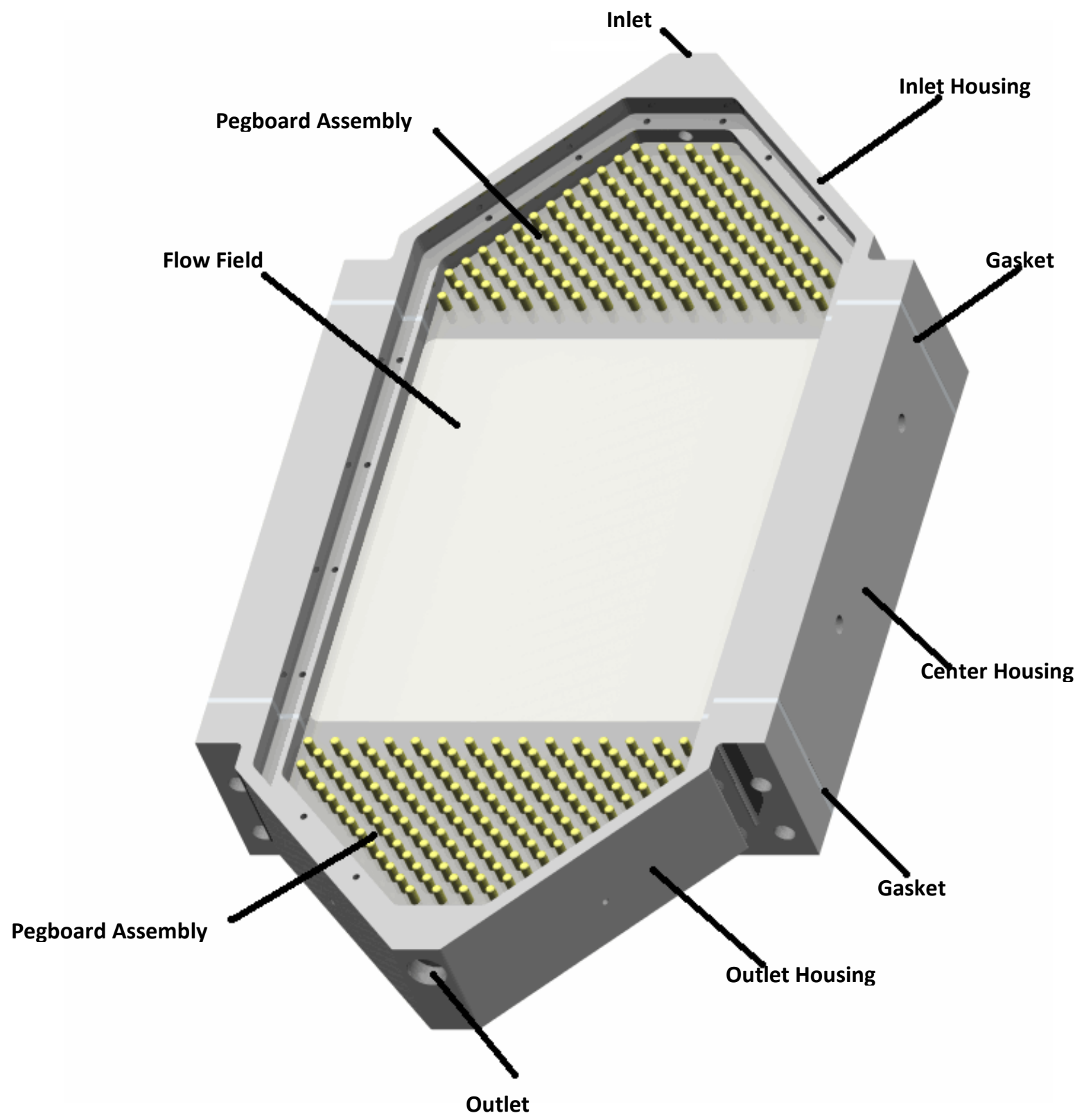

Figure 56: Collapsed view of final design for Modular Flow Field Test Fixture assembly 


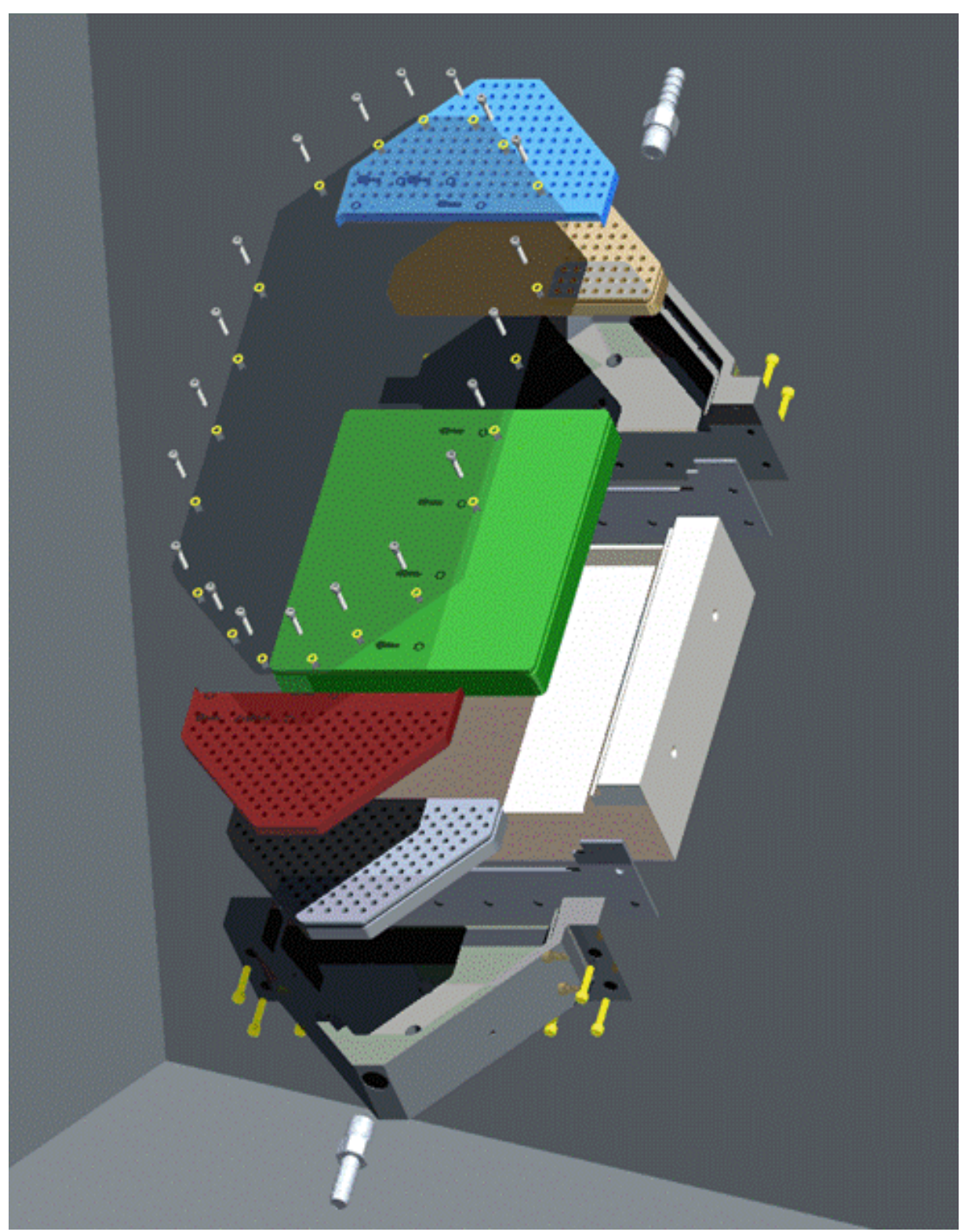

Figure 57: Exploded Modular Flow Field Test Fixture assembly.

The three pieces of the housing are connected by cap screws with a neoprene gasket separating each piece to prevent leaks. The three-piece assembly design allows for any shape of inlet and outlet housing to be fabricated and used in the fixture, therefore allowing for unlimited testing possibilities. It was recognized that having Pegboard Assemblies to manipulate flow at either the inlet, outlet or both was necessary to have the most capable test fixture. Also, the removable pegboard housing pieces allow for different pegboard shapes, such as rhomboidal or hemispherical, to be designed and implemented should we decide to test different flow field shapes. 
Currently, there exist two different shapes of Inlet and Outlet Housings. These are the triangular shape shown above, and a Square End Attachment, shown in Figure 58. The Square end attachment allows for the removal of flow manipulation at either end of the fixture, and allows for test configurations shown in Figures 59 and 60. Also, for testing and analysis purposes, the group designed a parallel flow field to observe the effects of a flow field instead of a blank plate. A 3D representation of the flow field can be found in Figure 61.

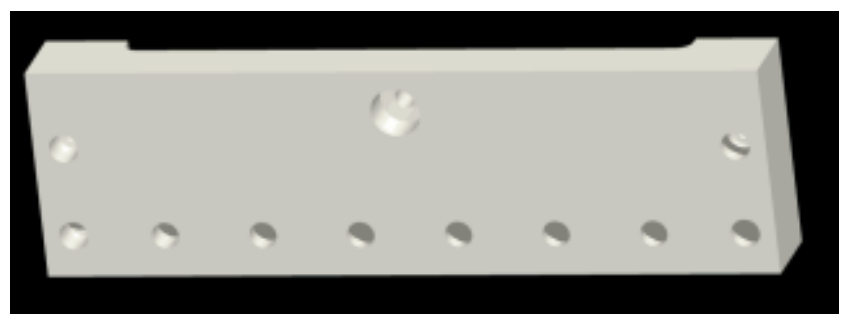

Figure 58: Test fixture square end attachment

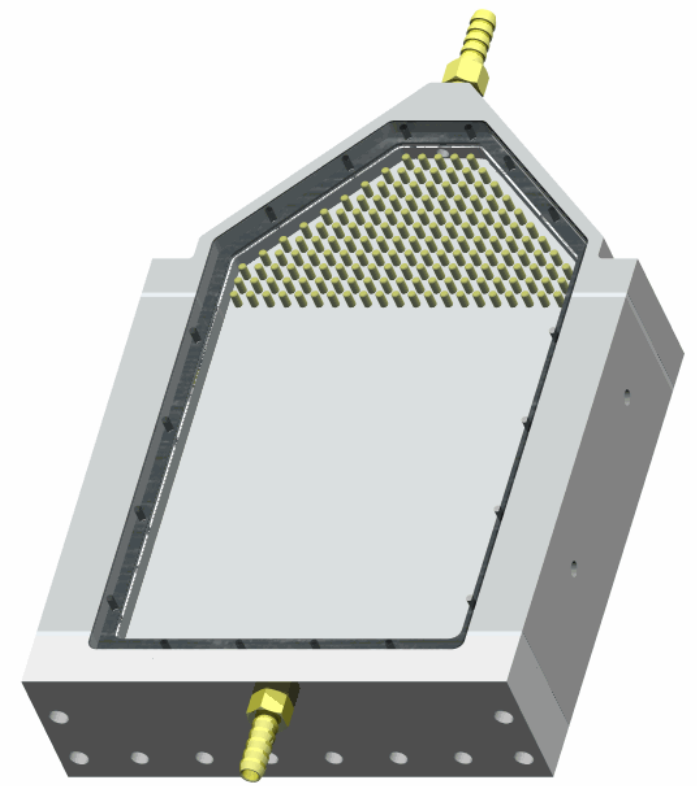

Figure 59: Modular flow field test fixture with square end attachment at outlet. 


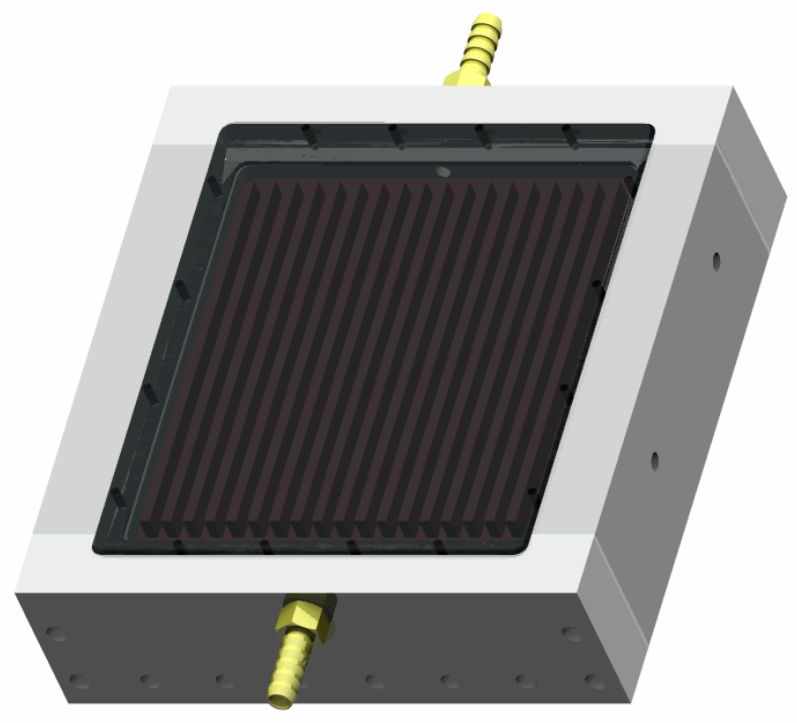

Figure 60: Modular flow field test fixture with square end attachments at inlet and outlet. Shown with parallel flow field inserted.

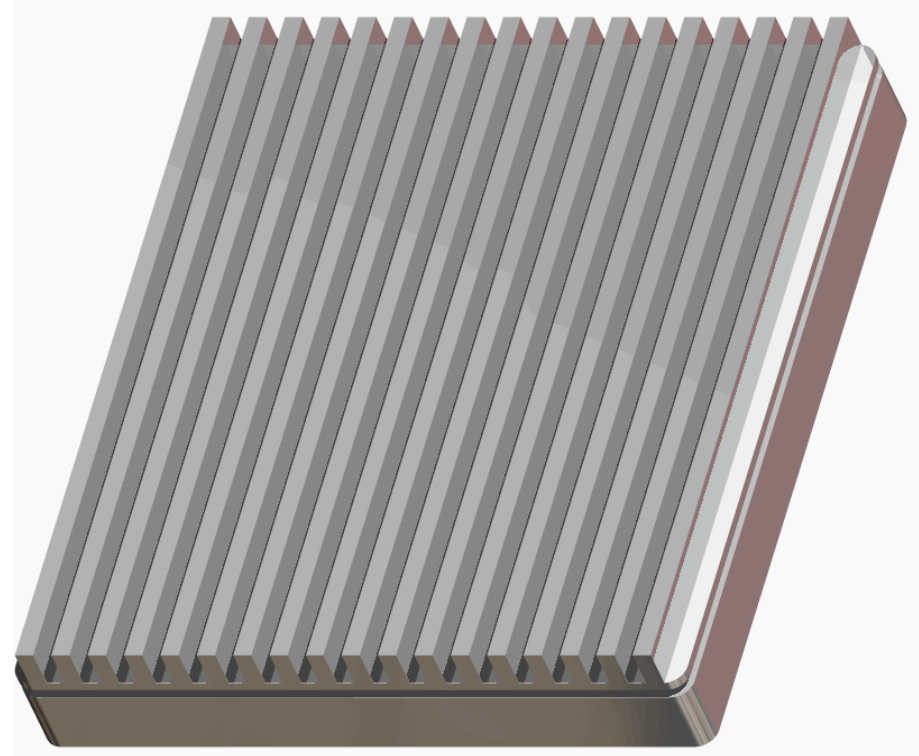

Figure 61: Parallel flow field.

\section{Test Procedure}

A test procedure has been developed incorporating the modular flow field test fixture. Water will be substituted for a methanol fuel mixture, as the methanol fuel used in the DMFC is highly diluted in water, making water an acceptable replacement. Water will be pumped into the sealed 
fixture using a syringe pump set to the prescribed flow rate. Once equilibrium flow conditions have been achieved, a syringe of water mixed with red dye will be placed in the syringe pump as a replacement for the original water-filled syringe. Meanwhile, a camera will be suspended directly above the fixture to record images of the flow patterns. A picture of the experimental setup can be found below in Figure 62 .

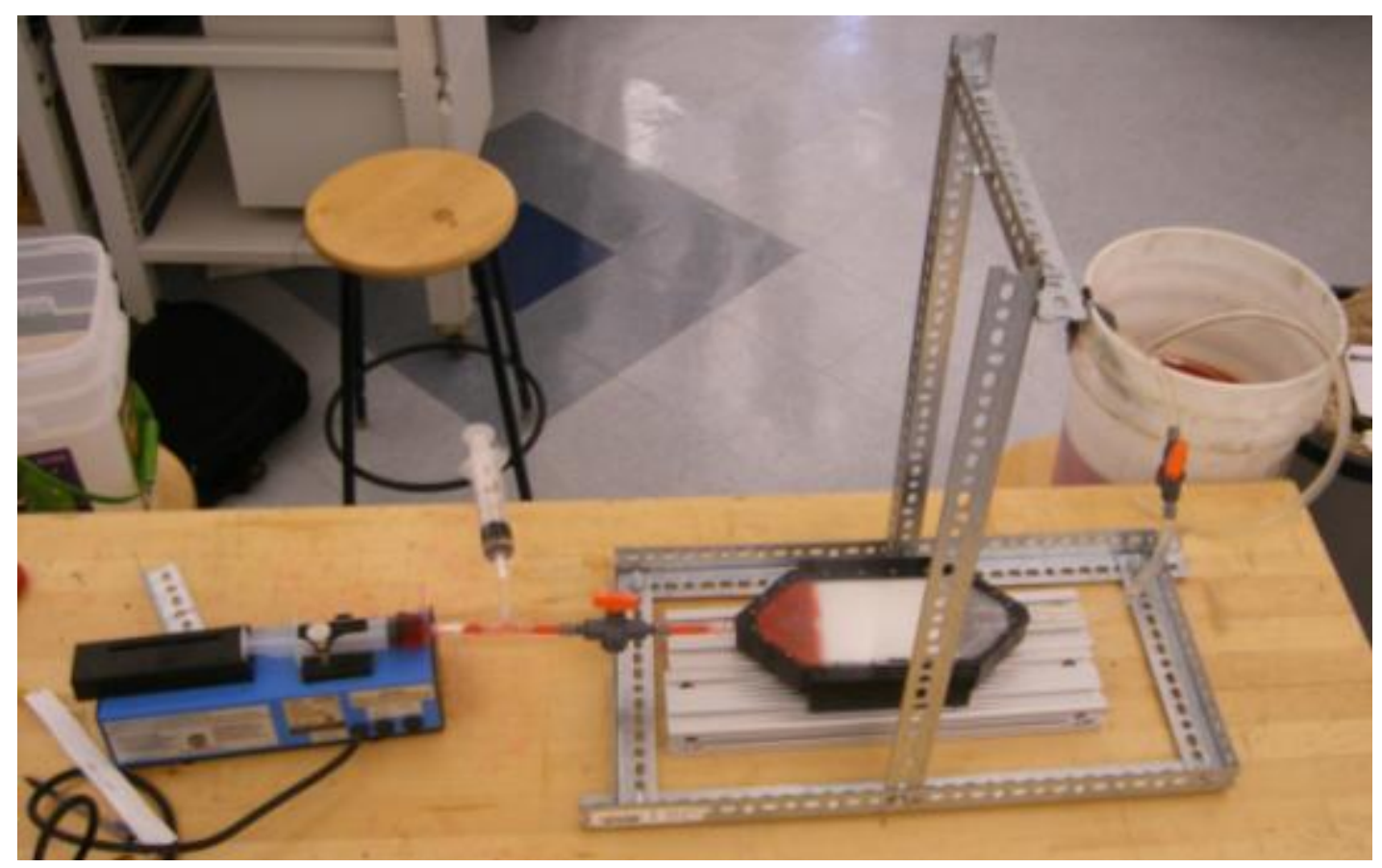

Figure 62: Experimental setup.

Once the dyed fluid begins to enter into the pegboard, the flow pattern of the stream will become readily visible due to the contrast between the dyed water and the white delrin background of the test fixture. The dye will clearly show the path of the flow and its velocity, and most importantly, the pattern by which it enters the flow field. Figure 63 shows photos of a test in chronological order which were run using the fixture. It is clear how visible the red dye is on the white background of the fixture, making for easy observational analysis and photo capture. 

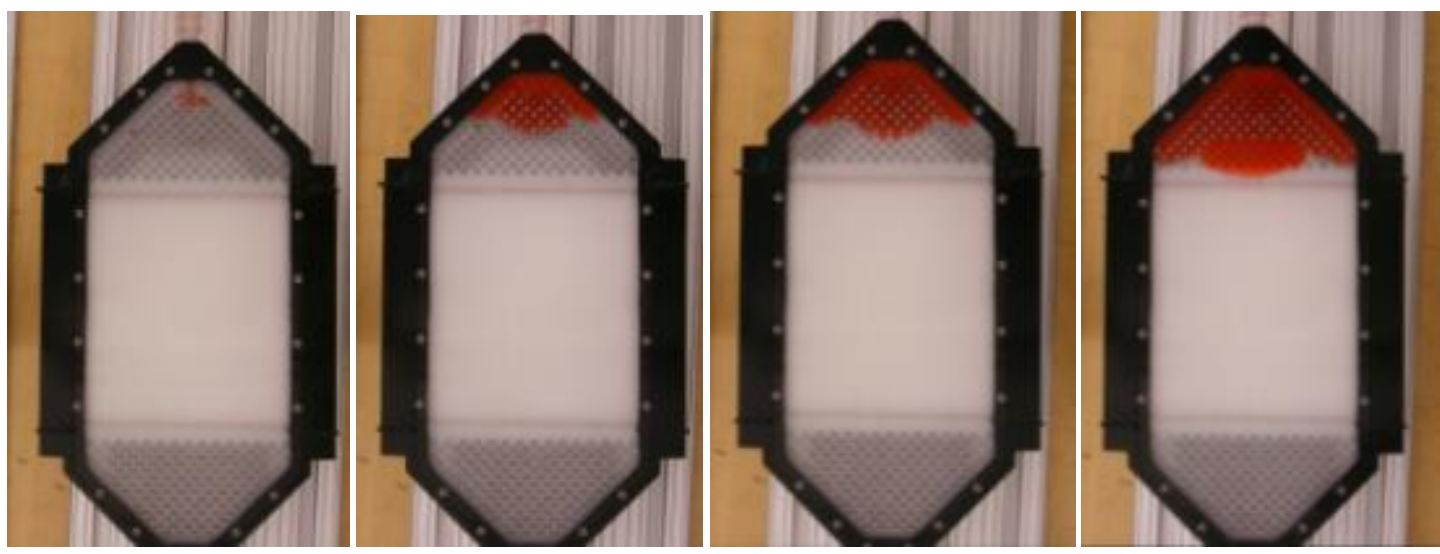

Figure 63: Modular Flow Field Test Fixture test sample.

In order to ensure the team only uses peg configurations that will be close to achieving uniform flow, analysis in Fluent, a program that analyzes fluid dynamics, will be done before each physical experiment. The results of Fluent will provide the team with a preview of how the flow will react to each peg configuration before the time is taken to set up an entire test run. If the team determines the flow pattern of a certain peg configuration does come close to meeting the uniformity requirement, then the team will verify the Fluent results by using the peg configuration in the test fixture.

\section{Peg Designs}

The design team felt the simple cylindrical dowel pins would not be sufficient in controlling the fluid flow pattern, so some peg attachments were designed. The five peg designs can be seen in Figure 64 below:

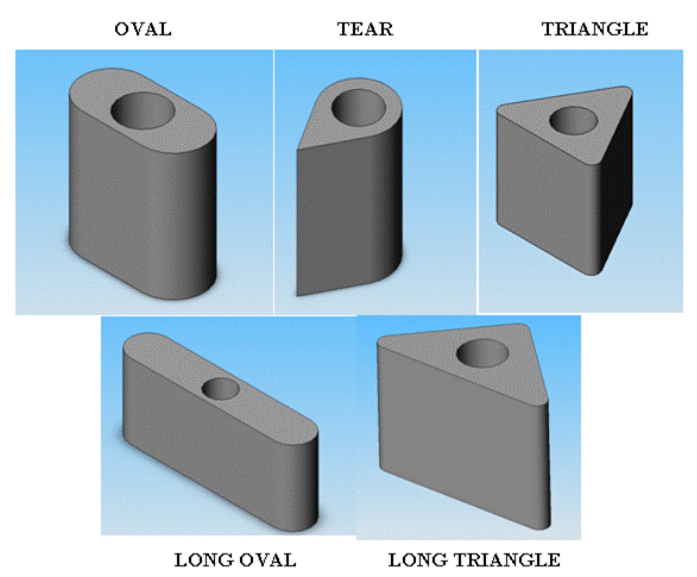

Figure 64: Peg attachments to be inserted into the

peg board of the modular flow field test fixture. 
Each peg is to be made out of aluminum rod stock, and have a hole through the center. The through hole is there so the addition can be attached and removed from the peg with ease, and also to make the fabrication easier for the machinist. Several of each attachment design will be machined to ensure the team does not run out of the different attachments when designing peg configurations. Figure 65 below shows an arrangement of these peg attachments inserted into the pegboard:

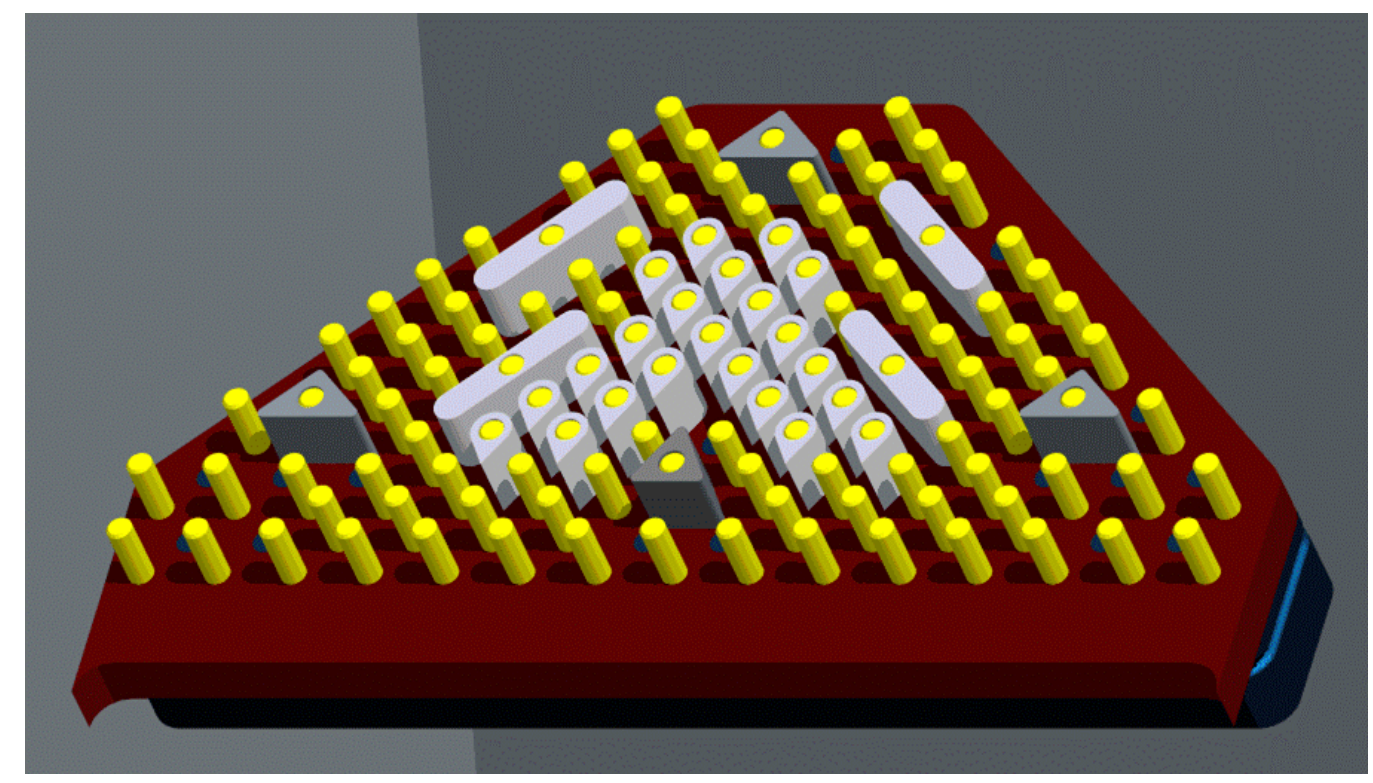

Figure 65: Screen shot of peg attachments inserted into pegboard of test fixture.

Unfortunately, due to time constraints, the team was unable to test any designs incorporating the shaped peg inserts. These pieces have been fabricated, but were received too late to allow for testing. They will be tested in future projects.

\section{Computational Analysis}

The design group used the computational fluid dynamics (CFD) modeling software Fluent to aid in the exploration of inlet and outlet configurations. Fluent is a powerful software program which applies the appropriate fluid dynamic equations to imported models. These models first go into a preprocessor program called Gambit, which creates the boundaries, surfaces, and meshes for Fluent applications. The models were created in Pro-Engineer Wildfire. IGES files were created of the 3D models to be imported into Gambit for preprocessing. 
After conducting a 3D and 2D comparative Fluent analysis it was concluded that 2D models of the test fixture were sufficient for data acquisition. The $2 \mathrm{D}$ representations were able to show us sufficient velocity vector and contour maps of the velocity magnitude. The depth of the preprocessing phase for 3D models was not justified based on the visibility of a $3 D$ model, shown below in Figure 66, compared to a 2D model, shown in Figure 67.

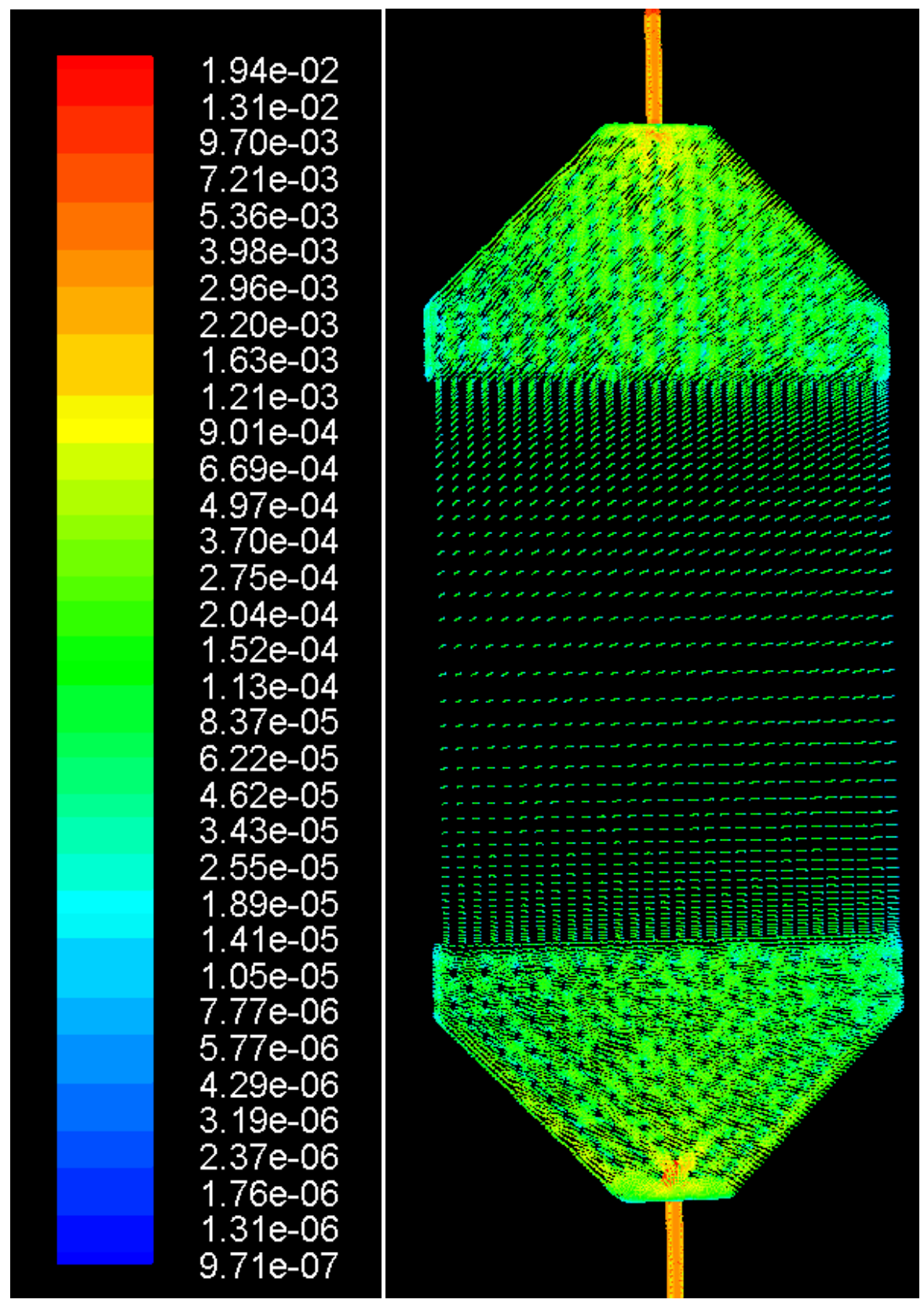

Figure 66: 3D Fluent display of velocity vectors (units in $\mathrm{m} / \mathrm{s}$ ) 

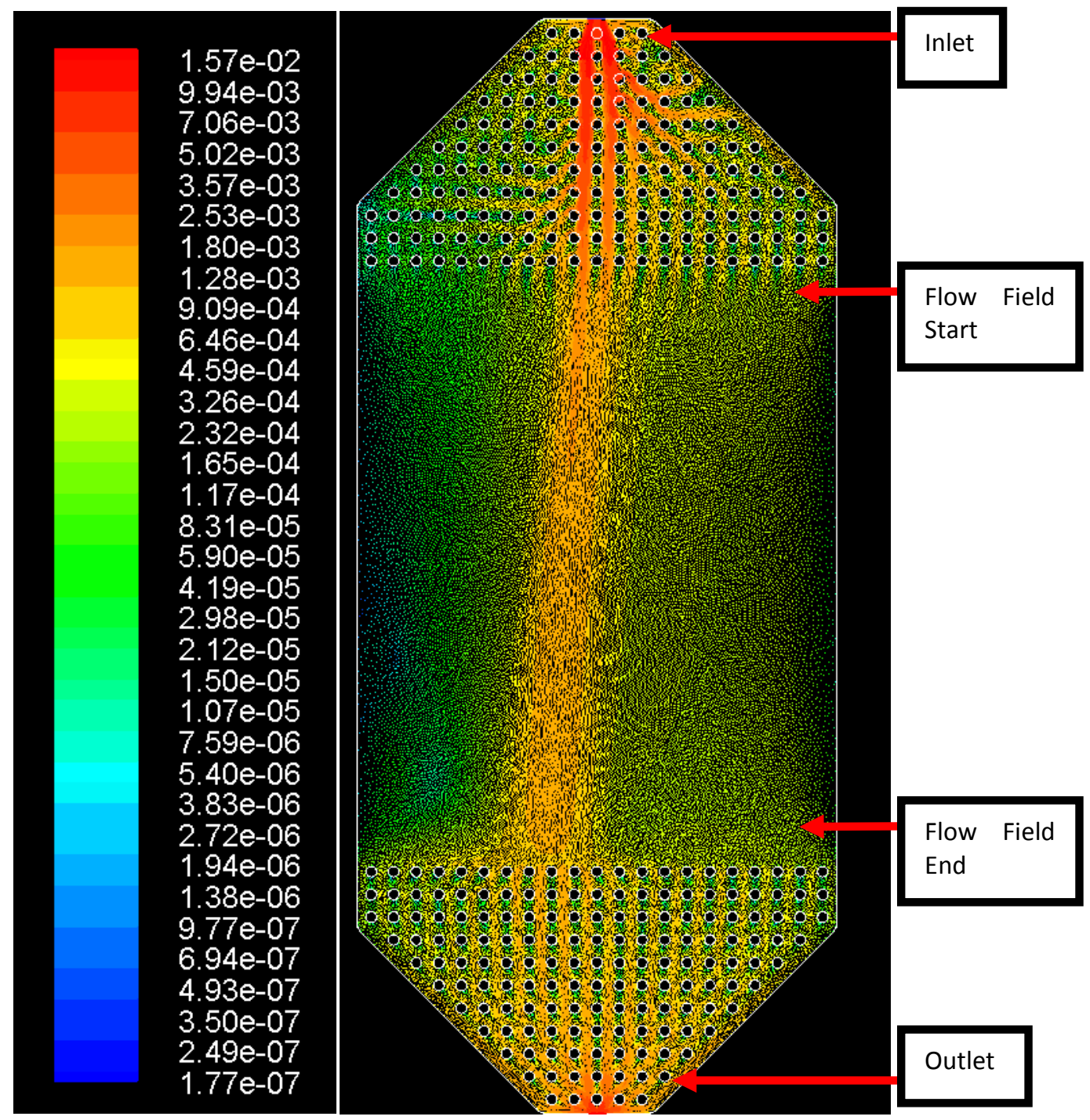

Figure 67: 2D Fluent display of velocity vectors (units in $\mathrm{m} / \mathrm{s}$ )

As seen in Figure 66, the 3D model does not add much clarity to the flow pattern display. It is easier to characterize the effects of individual peg configurations using the 2D display. Alterations to the peg configurations based on the 2D display lead to improvements in fluid uniformity entering the flow field area.

The 2D display criterion were based on velocity vector maps showing magnitude and direction and contour maps representing the velocity magnitude gradient. A promising pattern should show consistent magnitude velocity vectors leaving the peg field and entering the flow field section. The velocity vector map displays this by having a small color variation from the end of the peg field leading to the flow field section. This area is labeled in Figure 68 below. A consistent color is also desired horizontally between the side walls of the model as fluid moves 
through the flow field area. Figure 68 below shows a successful peg pattern whereas Figure 69 below shows an unsuccessful pattern.

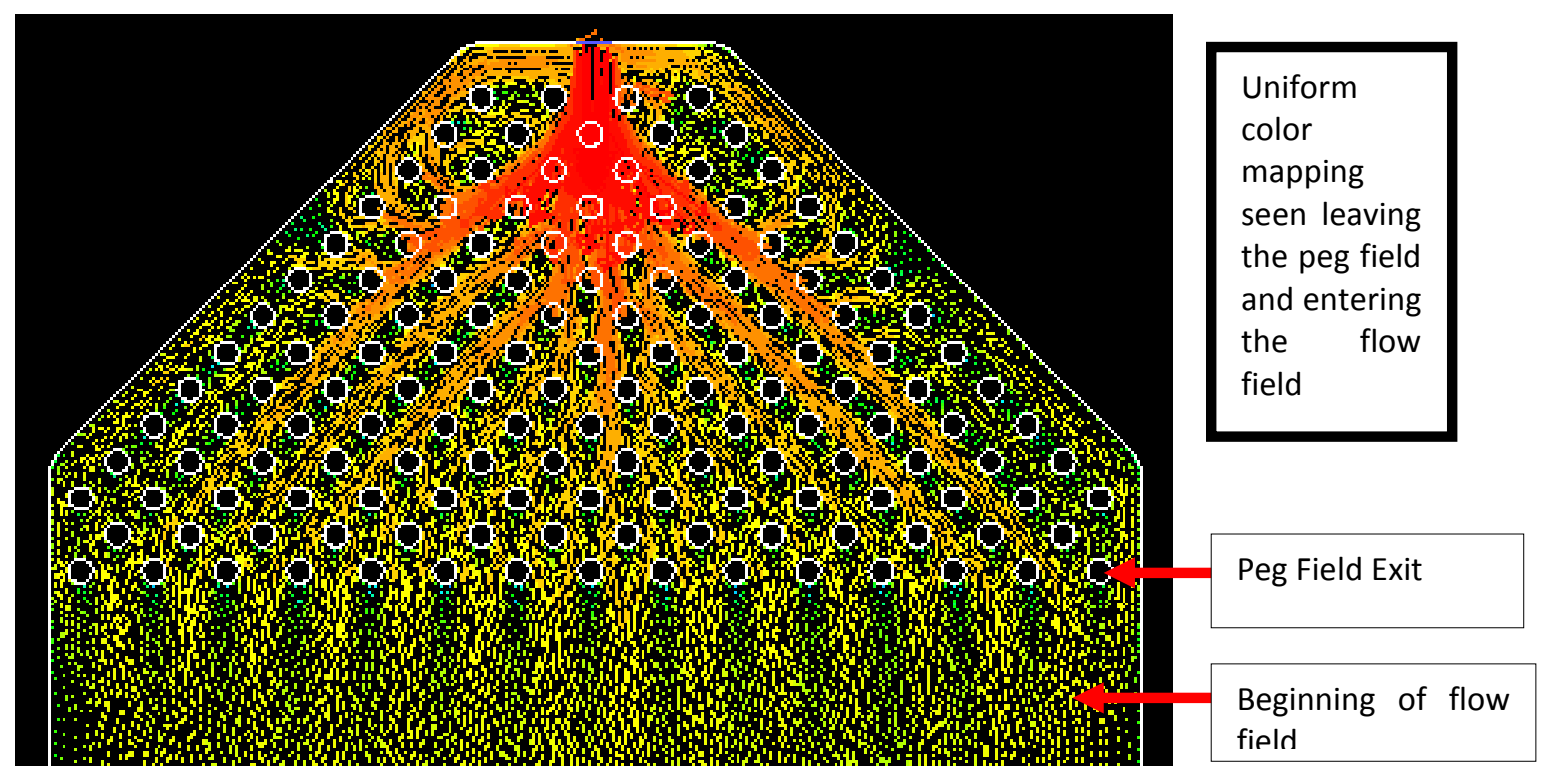

Figure 68: Vector map of velocity magnitude using Fluent representing a desirable uniform fluid flow.

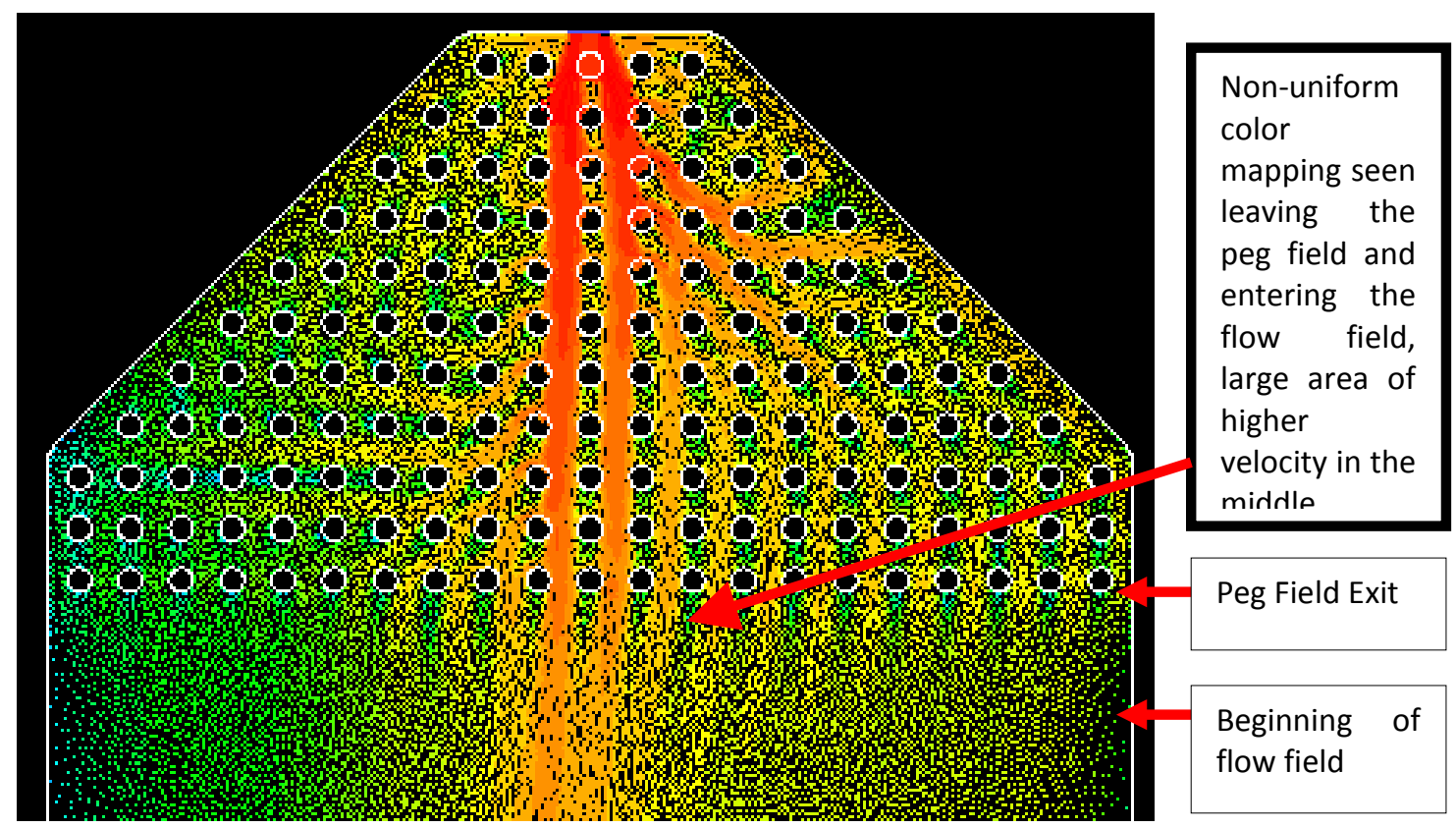

Figure 69: Vector map of velocity magnitude using Fluent representing an undesirable uniform fluid flow. 
A promising contour map shows consistent velocity in the field moving from a vertical centerline to the sidewalls. This is represented by small changes in color along the contour lines, which connect the inlet and outlet boundary. Figure 70 below shows a promising peg pattern with near linear contour lines within the flow field section, indicating a horizontal velocity profile which has little change as the flow travels further along the flow field. Figure 71 shows a poor peg pattern where erratic changes in velocity magnitude, at the labeled locations, demonstrate a nonuniform flow velocity as the fluid travels further down the flow field area.

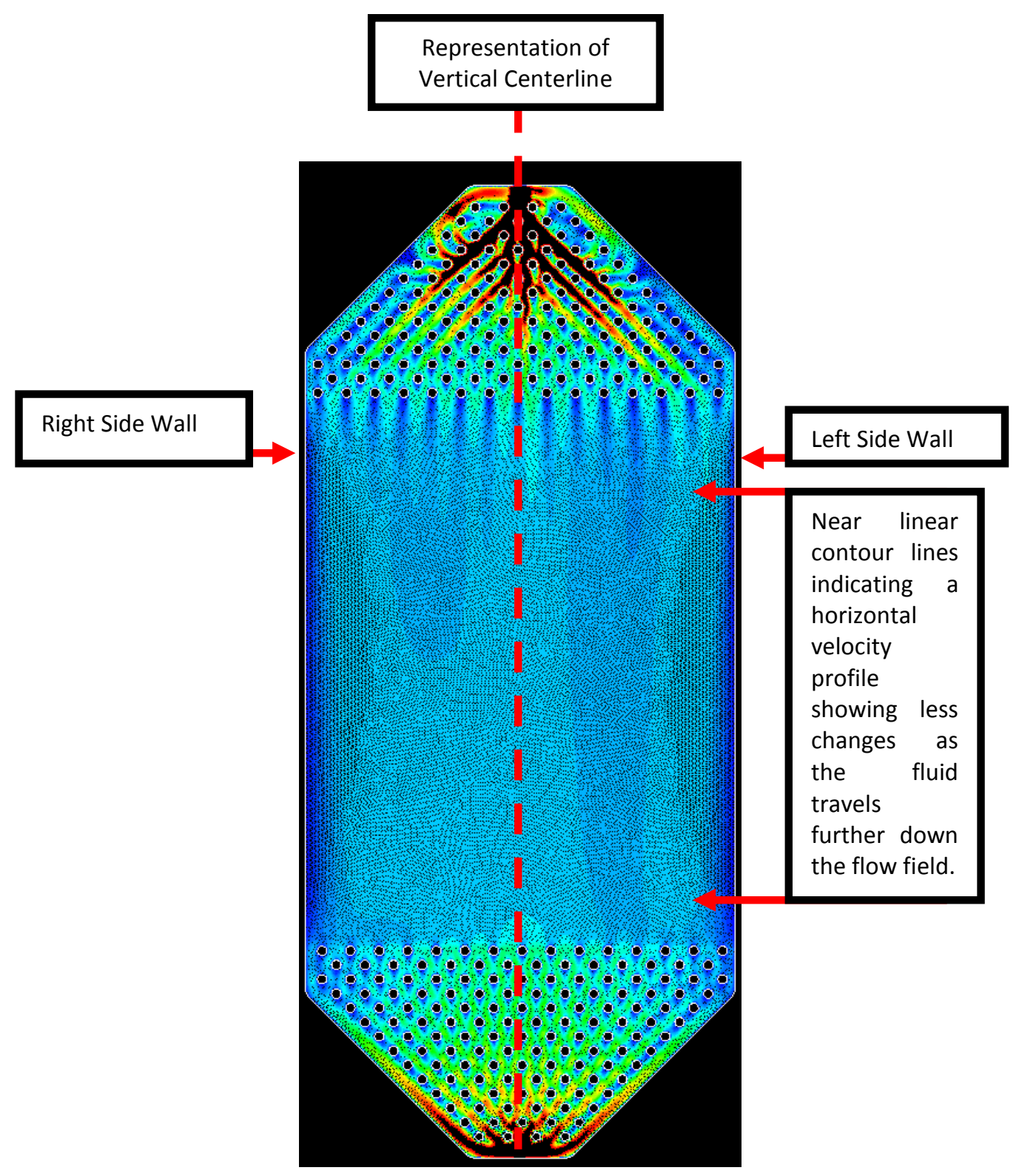

Figure 70: Contour map of velocity magnitude using Fluent representing a desirable uniform fluid flow. 


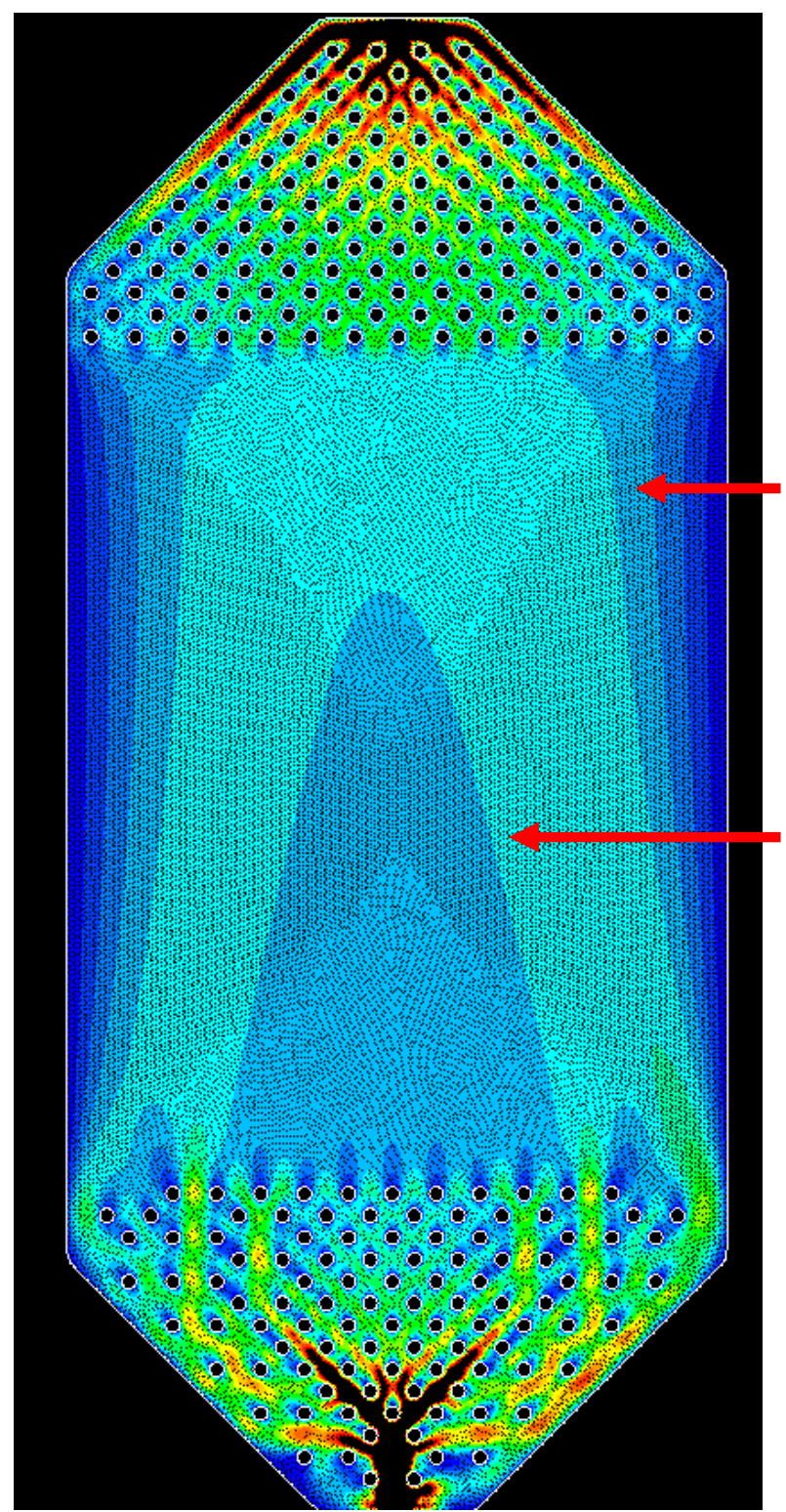

Converging contour lines showing the horizontal velocity profile changes as the fluid travels further down the flnw field

Figure 71: Contour map of velocity magnitude using Fluent representing an undesirable nonuniform fluid flow. 


\section{Experimental Analysis}

In order to verify the analysis produced using Fluent, the team used the modular flow field test

fixture to re-create the more successful peg configurations. The goal of the experiment was to mimic to results from Fluent, thus proving that the fixture gives an accurate portrayal of the Fluent analysis, as well as simulates the fluid flow within a fuel cell stack. This section will show how the team decided which peg configurations to test, and how the testing results contributed to the design of other peg configurations. The peg configurations were given labels based on their geometry. If a peg configuration had a diamond peg board on the inlet side and a square peg board on the outlet side, it was given the label Square Diamond (SD). After the geometry label, each configuration was given a number. For example, the $3^{\text {rd }}$ peg configuration that was designed with a diamond peg board at both the inlet and outlet was given the label DD3.

\section{Fluent Verification}

The first test was done with the purpose of proving the test fixture verifies the Fluent analysis. The team chose to run a test that showed non-uniformity across the flow field. This particular peg configuration, which the team called SS1, displayed a velocity contour map where the velocity magnitude was greater in the center of the peg field than on the sides. This contour map presented the conclusion that the fluid would flow in a parabolic shape down the flow field. Figure 72 shown below gives a side by side comparison view of the velocity contour map and an image taken during the testing of the SS1 configuration: 

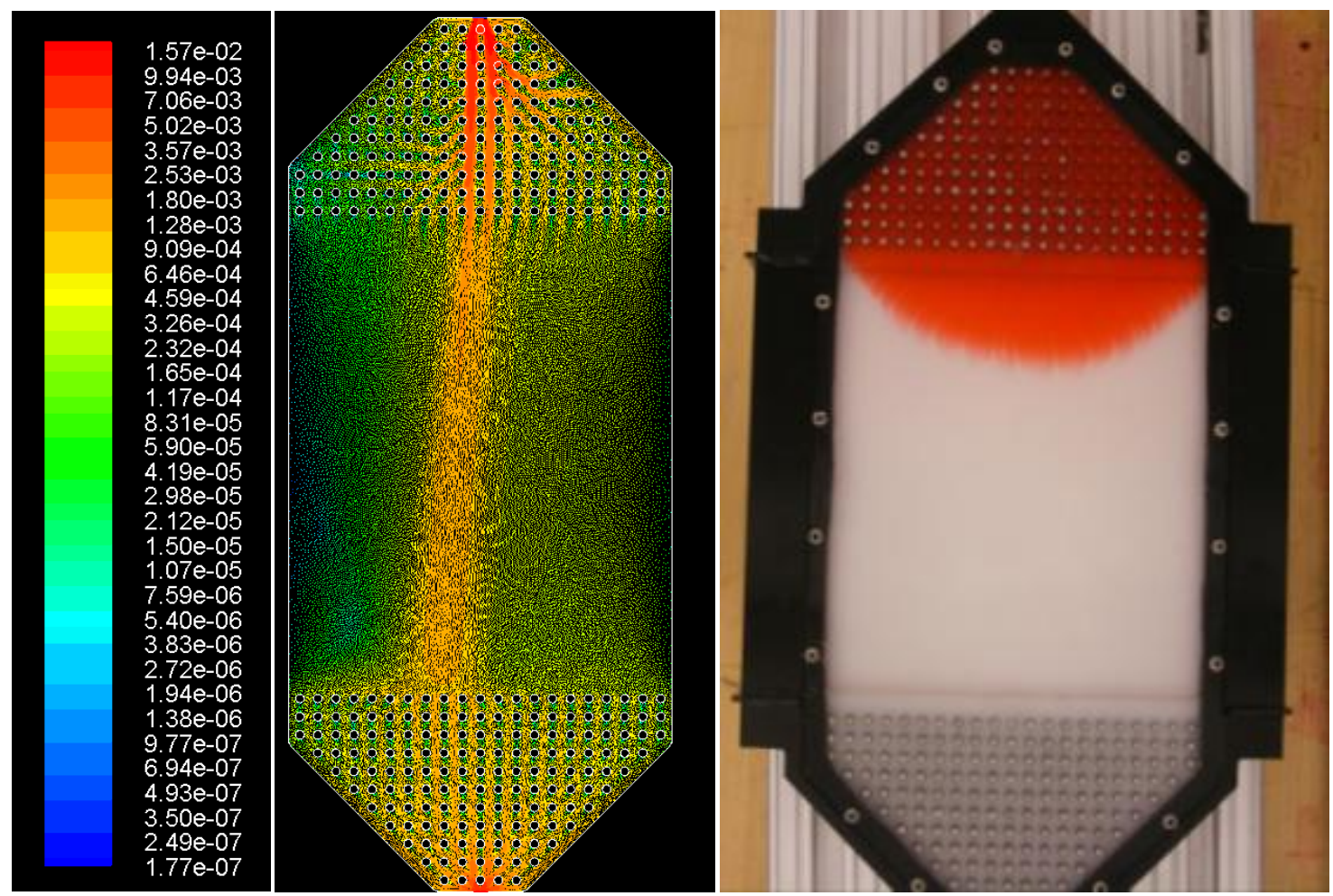

Figure 72: Side by side comparison of Fluent analysis and test run of SS1 (units in m/s).

The orange contours of the Fluent screen shot show that the fluid is moving at a speed of about $3.57 \mathrm{e}-3 \mathrm{~m} / \mathrm{s}$. This is faster than the speed of the fluid to at the sides of the fluid indicated by the green contours. The fluid to the sides of the test fixture is moving at about $1.17 \mathrm{e}-4 \mathrm{~m} / \mathrm{s}$. This data should indicate that the fluid in the center of the fixture should reach to flow field area first during experimental testing. The picture of the test procedure clearly shows how the experimental analysis of SS1 shows the same results as the computational analysis. The team was able to determine that the Fluent analysis and the experimental analysis were nearly identical.

There were some possible sources of error which were noticed during the experimental analysis. For one, any shaking of the fixture can result in inconsistent data. Also, a less than perfectly level surface upon which to mount the test fixture can cause visible changes in flow patterns. Also, failure to properly flush the system of air at the start of a test can cause an invalid test. These problems were bypassed successfully during testing.

\section{Peg Configuration Adjustment.}

The results from each experiment were used to assist the team in designing new peg configurations. The team used these results by altering peg configurations based on how the 
fluid entered the flow field area. If the peg configuration was providing too much resistance in a certain area of the peg field, then the team altered the configuration to try to achieve uniform flow at the start of the flow field area. Figure 73 below shows the picture from the test of peg configuration DD9 along with a screen shot of the peg configuration as seen in Gambit.
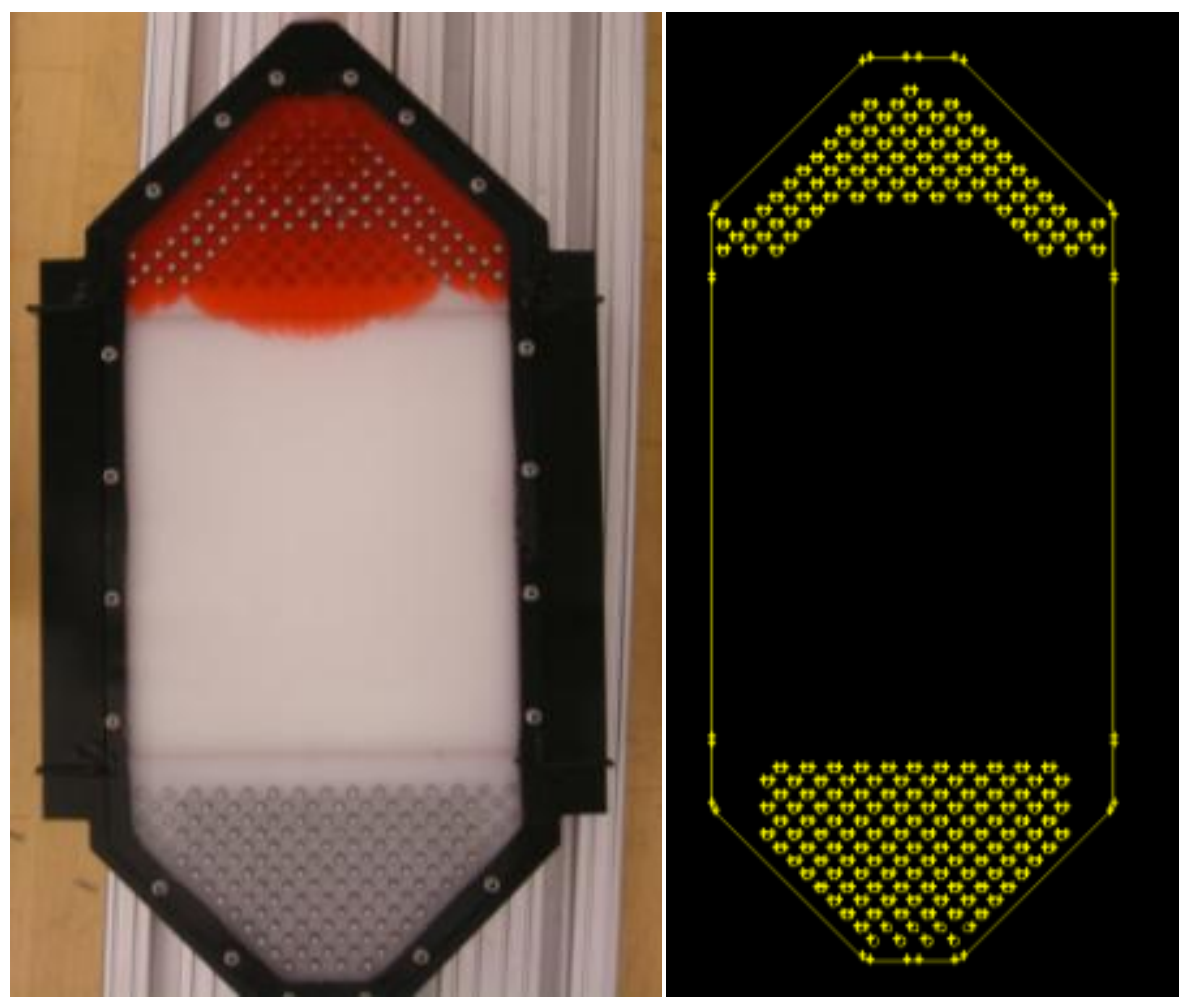

Figure 73: Picture of DD9 during testing along with a screen shot from Gambit showing the peg configuration.

As the picture shows, the fluid was encountering too much resistance on the sides of the peg field, which caused the fluid in the middle of the peg field to reach to flow field area first. While this design yielded non-uniform flow, this test served as a building block for other peg configuration designs. 


\section{Results}

The team determined that two of the designed peg configurations met the goal of achieving uniform flow at the edge of the peg field. One design uses a square peg configuration at the inlet, while the other uses a diamond peg configuration. Those designs are DD11 and SS8. The designs were achieved after studying several iterations of each peg configuration type (diamond or square) and the team feels the chosen designs will provide a reliable flow field for the fuel cell stack

\section{Peg Configuration DD11}

Design DD11 was created based on testing and Fluent analysis of DD9, which was explained in the previous section. During the test run of DD9, the team determined that pegs needed to be removed from the side areas of the peg field to ensure that the fluid on the sides reached the flow field area sooner. Figures 74 below shows a side by side comparison of the Gambit screen shot of the inlets for peg configurations DD9 and DD11.
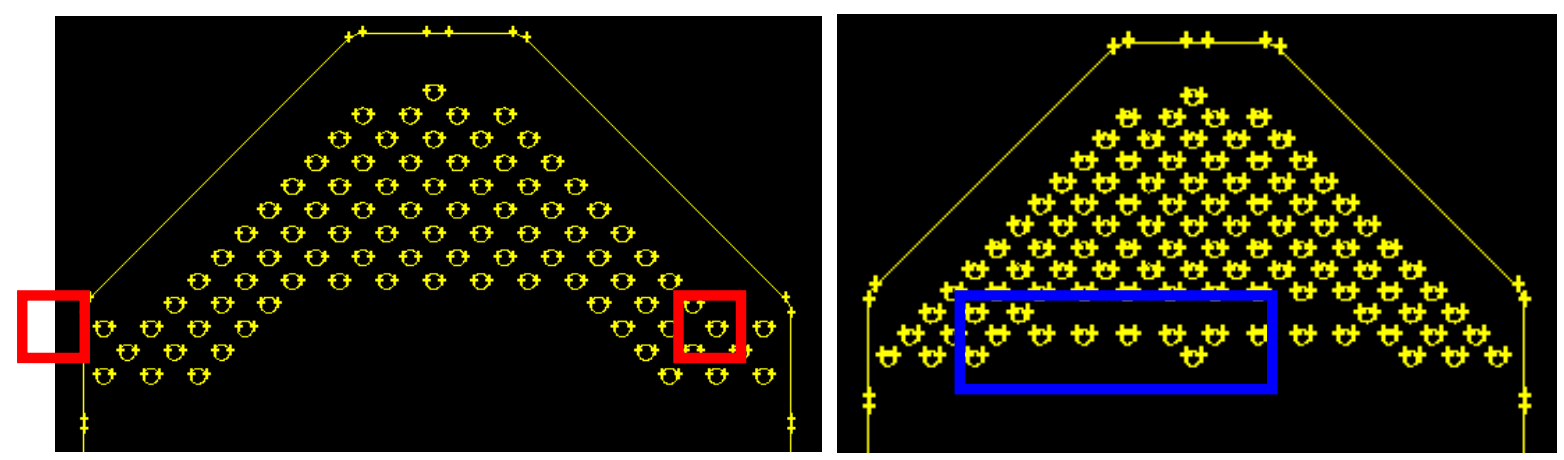

Figure 74: Side by side comparison for the peg configurations DD9 and DD11. The RED boxes indicate pegs that were removed from peg configuration DD9. The BLUE box indicates pegs that were added to the configuration, creating DD11.

While the team determined that pegs needed to be removed from the side of the peg board to speed up the fluid flow along the sides of the fixture, the team also added a row of pegs in the center of the peg field in order to provide resistance and hopefully even out the flow velocity. The comparison photos show that the alteration to the peg configuration had a definite impact on the fluid flow. Adding resistance to the middle of the peg field, while eliminating resistance at the sides of the peg field achieved a more uniform flow at the start of the flow field area. Before the altered peg configurations are tested, these configurations are analyzed using Fluent to give the tester an idea what to expect during the test. Figure 75 is the velocity magnitude contour 
map of DD11. The velocity contours show that the fuel is flowing faster along the sides of the peg field, allowing it to leave the peg field at the same time as the fluid towards the center.

Near linear

contour

lines

indicate a

horizontal

velocity

profile

respectively

connecting

both $A$ and $B$

pointers.
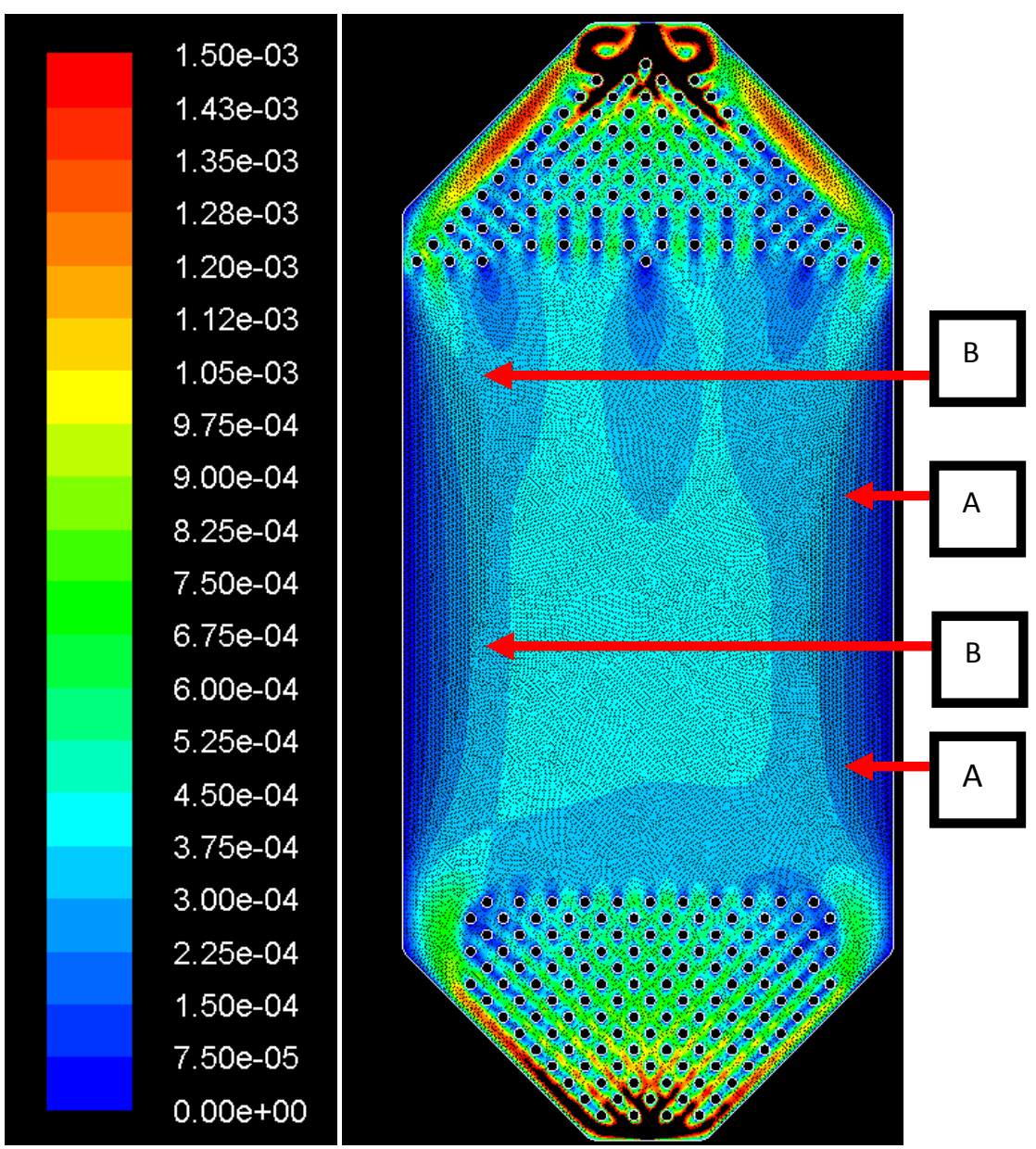

Figure 75: Velocity Magnitude Contour Map of DD11 (units in m/s)

According to this map, the fluid should be more uniform once if leave the peg field, as opposed to the fluid flow from configuration DD9. Figure 76 below shows a side by side comparison of the inlet for both DD9 and DD11 during testing: 

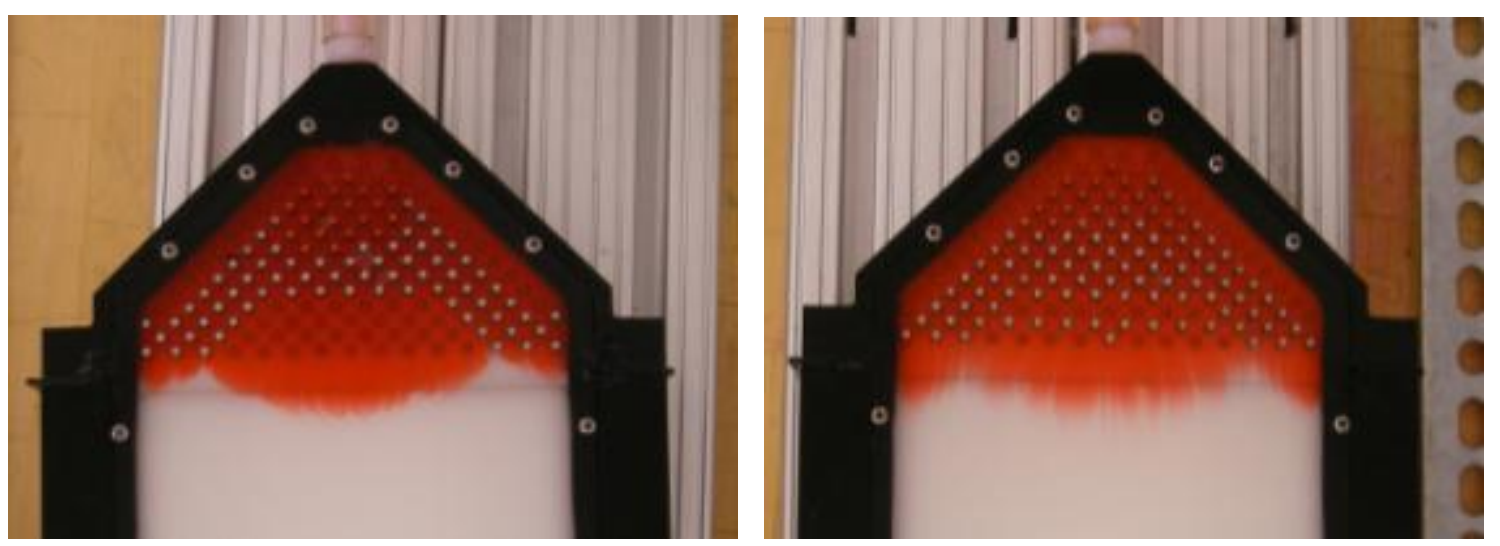

Figure 76: Side by side comparison of DD9 (left) and DD11 (right) during testing. Picture is taken just as the fluid reaches the start of the flow field area.

As shown in the figure, the adjustment of the peg configuration produced a more uniform fluid flow. The slow moving fluid on the sides of the peg field was allowed to flow faster by removing only 1 peg from each side. The addition of a row of pegs in the middle forced the fluid flow in the middle to slow down, thus yielding a near uniform fluid flow at the start of the flow field region.

\section{Peg Configuration SS8}

Peg configuration SS8 proved to be the most successful at achieving uniform flow at the start of the flow field region. SS8 uses the square peg board assembly at both the inlet and outlet. Figure 77 below is the velocity magnitude contour map of SS8. 


Near linear
contour lines
indicate a
horizontal
velocity
profile
respectively
connecting
both A and B
pointers.

Near linear contour lines indicate a horizontal velocity profile respectively connecting pointers.

Figure 77: Velocity Magnitude Contour Map of SS8 (units in $\mathrm{m} / \mathrm{s}$ )

The figure shows that the velocity of the fluid along the sides of the fixture is slightly faster than the fluid in the middle. This is due to less resistance from pegs on the sides. Faster flow on the sides allows the fluid to travel along the peg field and converge on the flow field area at the same time as the fluid in the middle. The flow on the sides needs to be slightly faster due to the position of the inelt. With the inlet aligned in the center of the fixture, the fluid flowing through the middle of the peg field has a shorter distance to travel than the fluid that has been dispersed to the sides. Less resistance on the sides helps the fluid travel that longer distance faster. Below is a picture from the test run of SS8: 


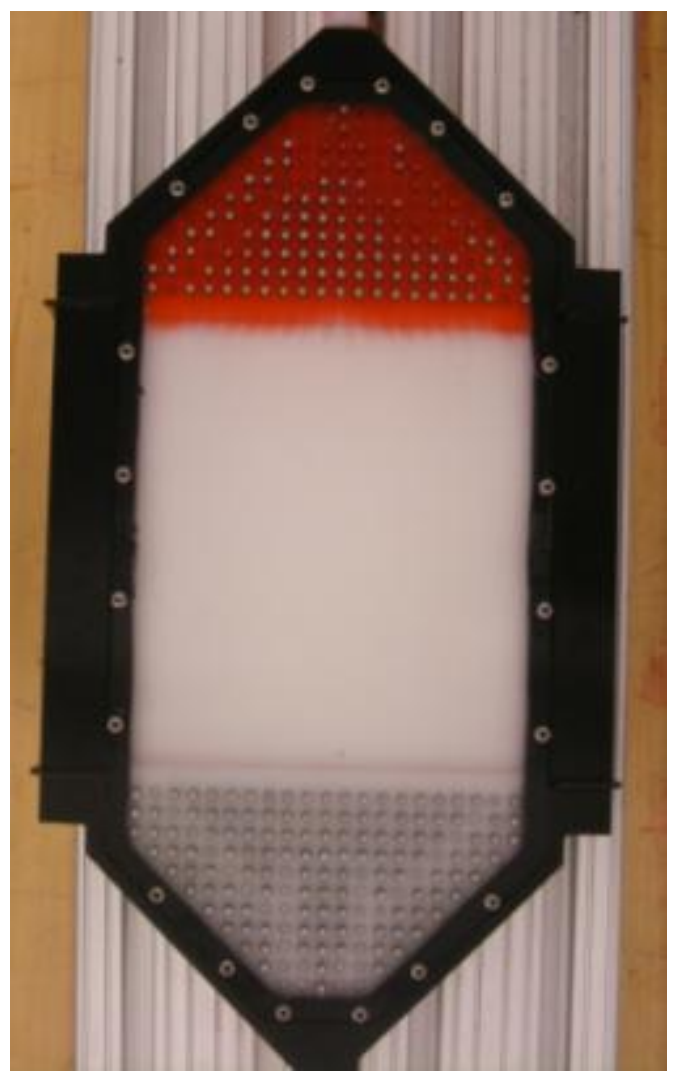

Figure 78: Picture during test of SS8. Shows the uniformity of the fluid flow just as it approached the flow field area.

As seen in the figure, the flow has achieved uniformity as it approaches the beginning of the flow field area. The absence of pegs allows the fluid on the side to flow faster, and arrives at the edge of the peg field at the same time as the fluid in the middle of the peg field, similar to design DD11. These two peg configurations will be presented to NuVant as design possibilities for their future flow field prototypes. Flow uniformity like this could potentially lead to a reliable energy output from the fuel cell stack, since the fluid flow allows for a complete chemical reaction along the fuel path. 


\section{Conclusion}

Currently, the new modular flow field test fixture has passed the latest design review held by the design team and is ready for fabrication. Once the test fixture is completed and all of the necessary hardware has been collected, the team will build the test apparatus to start experimenting with different flow field and peg configurations. The team believes they have designed a fixture and test procedure that will yield sufficient data about the flow pattern of the reactants across the DMFC flow fields. Using this data, the team will then begin the task of designing a new flow field to be incorporated into a future DMFC stack. A good peg configuration is an important component of any flow field, and coming up with successful designs of configurations would be deemed a success for the project. Future work needs to be done with water management and heat regulation within the flow field, as well as the entire stack. 


\section{Milestone\#8: Step Impedance Spectroscopy. Design and operation.}

Impedance spectra are routinely used to study the electrode reactions in fuel cells by application of sinusoidal perturbations at fixed frequencies, over a range of frequencies from $\mathrm{mHz}$ to $\mathrm{MHz}$. One of the drawbacks in that experimental approach is the time needed for the whole test may exceed the time for which the system goes significant changes. An alternative is to use an excitation signal that consists of a multiple sine-waves with analysis of the response by fast Fourier transformations (FFT). The total measurement time is equal to the time required for the lowest frequency used. This approach requires the selection of frequencies with nonoverlapping second harmonics, which puts additional complexity to the used signal generator. Additionally the measurements are more sensitive to the noise present in the system.

To eliminate the need of a complex signal generator we have chosen to use an alternative approach by applying a current pulse as the excitation signal. The response signal then is analyzed in the time domain for common electrochemical cell equivalent circuit models. Signal transient along with the boundary values associated with the start and end of the excitation signal, provides physically relevant values of the model parameters from the fit of the timedomain data.

Theoretical background for use Step Impedance Spectroscopy (SIS®)

Glossary of Abbreviations for SIS ${ }^{\circledR}$

$\mathrm{t}_{0} \quad$ Beginning of the pretreatment period.

$t_{p, i} \quad$ Pulse initiation time (end of pretreatment period)

$t_{p, f} \quad$ Pulse final time

$\mathrm{t}_{\mathrm{r}, \mathrm{f}} \quad$ End of relaxation time

$t_{p w}=t_{p, f}-t_{p, i} \quad$ Pulse width

$t_{r p}=t_{r, f}-t_{p, f} \quad$ Relaxation period

$E(t)=E_{\text {eq1 }} \quad$ For $\left(t_{p, i}-t_{\text {interv }} \leq t \leq t_{p, i}\right)$

$\mathrm{E}_{\text {eq2 }} \sim \mathrm{E}\left(\mathrm{t}_{\mathrm{r}, \mathrm{f}}\right)$

$\mathrm{T} \quad \mathrm{RC}$ time constant specific to differential equation

$i_{p} \quad$ Pulse amplitude

tinterv Sampling interval time

RE Reference electrode

WE Working electrode 


$\begin{array}{ll}\text { CE } & \text { Counter electrode } \\ \text { Cd } & \text { Double layer capacitance } \\ \text { Cc } & \text { Capacitance of coating } \\ \text { Rs } & \text { Series resistance } \\ \text { Rcp } & \text { Porous coating resistance } \\ \text { Zw } & \text { Warburg impedance } \\ \text { Cw } & \text { Capacitive component of Warburg impedance } \\ \text { Rw } & \text { Resistive component of Warburg impedance } \\ \text { Rct } & \text { Charge transfer resistance } \\ \text { ZD } & \text { Mass-transport element }\end{array}$

Randles cell

This model consists of parallel-connected resistance-capacitance circuit put in series with a resistance Error! Reference source not found.

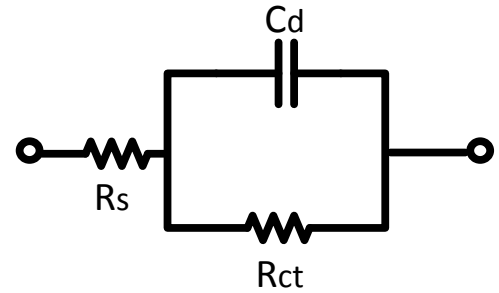

Figure 79: Randles Cell

The current and voltage measured at the ends of the circuit are governed by the following differential equation:

$R_{c t} C \frac{d V}{d t}+V=I\left(R_{s}+R_{c t}\right)+R_{s} R_{c t} C \frac{d I}{d t}$

When a constant current $(\mathrm{dl} / \mathrm{dt}=0)$ is applied to the Randles Cell circuit the above equation can be simplified to:

$\frac{d V}{d t}+\frac{V}{R_{c t} C}=\frac{i\left(R_{s}+R_{c t}\right)}{R_{c t} C}$ 
The solution of this equation is:

$\frac{i\left(R_{s}+R_{c t}\right)}{R_{c t} C} V=R_{c t} C+A \times e^{-\frac{t}{R_{c t} C}}$

When the current is applied as a current pulse from 0 to a non zero value $i_{p}$, Figure 80 . Rectangular current pulse ip=-1mA applied to a Randles cell., the solution in the region with non zero current will be:

$V(t)=i_{p} R_{s}+i_{p} R_{c t}\left(1-e^{-\frac{t}{R_{c t} C}}\right)$

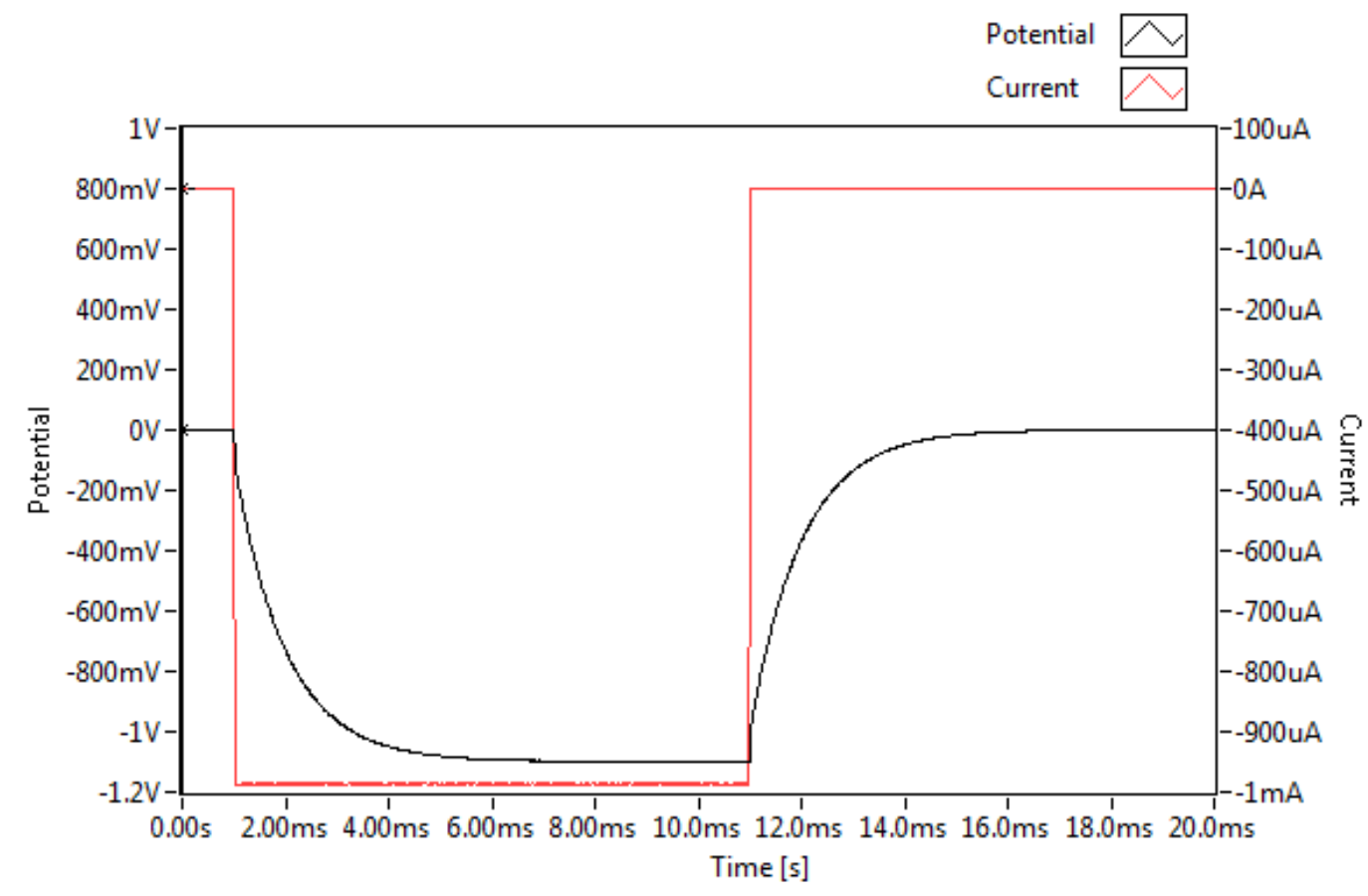

Figure 80. Rectangular current pulse $\mathrm{i}_{\mathrm{p}}=-1 \mathrm{~mA}$ applied to a Randles cell.

When the current returns from $i_{p}$ to 0 the solution is:

$V(t)=\left(V_{0}-i_{p} R_{s}\right) e^{-\frac{t}{R_{c t} C}}$

If the time of the non-zero pulse is long enough, the voltage to reach equilibrium in the above equation can be simplified further to:

$V(t)=i_{p} R_{c t} e^{-\frac{t}{R_{c t} C}}$ 
These equations can be used to fit the experimental $V(t)$ curve and extract the values of $R_{s}, R_{c t}$, and $C$ from the fitting parameters.

\section{Battery analysis}

Case 1.

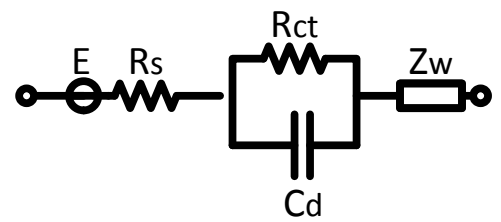

Figure 81. Modified Randles cell for battery electrode. $\mathrm{E}(\mathrm{t})$ : battery voltage, Rs: solution resistance, Cd: double layer capacitance, Rct: charge transfer resistance, Zw: Warburg impedance (i.e., diffusion phenomenon)

The differential equation for this circuit can be written as:

$R_{c t} C_{d} \frac{d V}{d t}+V=I\left(R_{s}+R_{c t}\right)+R_{s} R_{c t} C_{d} \frac{d I}{d t}+\left(E_{e q 1}+\frac{d E_{e q}}{d Q} I t\right)+Z_{w}(t)$

If we approximate $Z_{w}$ with $Z_{w}(t)=\frac{1}{S_{0}}$ It the equation can be rewritten:

$R_{c t} C_{d} \frac{d V}{d t}+V=I\left(R_{s}+R_{c t}\right)+R_{s} R_{c t} C_{d} \frac{d I}{d t}+E_{e q 1}+\left(\frac{d E_{e q}}{d Q}+\frac{1}{S_{0}}\right) I t$

When a rectangular current pulse is applied to the system the differential equation yields the following two solutions:

$V(t)=\left[i_{p} R_{s}+i_{p} R_{c t}\left(1-k C_{d}\right)+E_{e q 1}\right]+k i_{p} t+i_{p} R_{c t}\left(k C_{d}-1\right) e^{-\frac{t}{R_{c t} C_{d}}} \quad$ When $I=i_{p}\left(\mathrm{t}_{\mathrm{p}, \mathrm{i}} \leq \mathrm{t} \leq \mathrm{t}_{\mathrm{p}, \mathrm{f}}\right)$

And

$V(t)=E_{\text {eq2 }}+\left(E_{\text {eop }}-E_{\text {eq } 2}-i_{p} R_{s}-\frac{i_{p} i_{p w}}{S_{0}}\right) e^{-\frac{t}{R_{c t} C_{d}}} \quad$ When $I=0\left(\mathrm{t}_{\mathrm{p}, \mathrm{f}} \leq \mathrm{t} \leq \mathrm{t}_{\mathrm{r}, \mathrm{f}}\right)$ 
Where: $k=\frac{\Delta E_{e q}}{\Delta Q}+\frac{1}{s_{0}}$ and $Z_{w}(t)$ and $E_{e q}(t)$ vary linearly with t during short current pulses.

EZware uses $E_{e q}$ before the pulse $\left(E_{e q 1}\right)$ and after relaxation $\left(E_{e q 2}\right)$ to define $E_{e q}(t)$.

The parameters used in this analysis are illustrated in Figure 82. Parameter definition in SIS for battery and fuel cell systems.

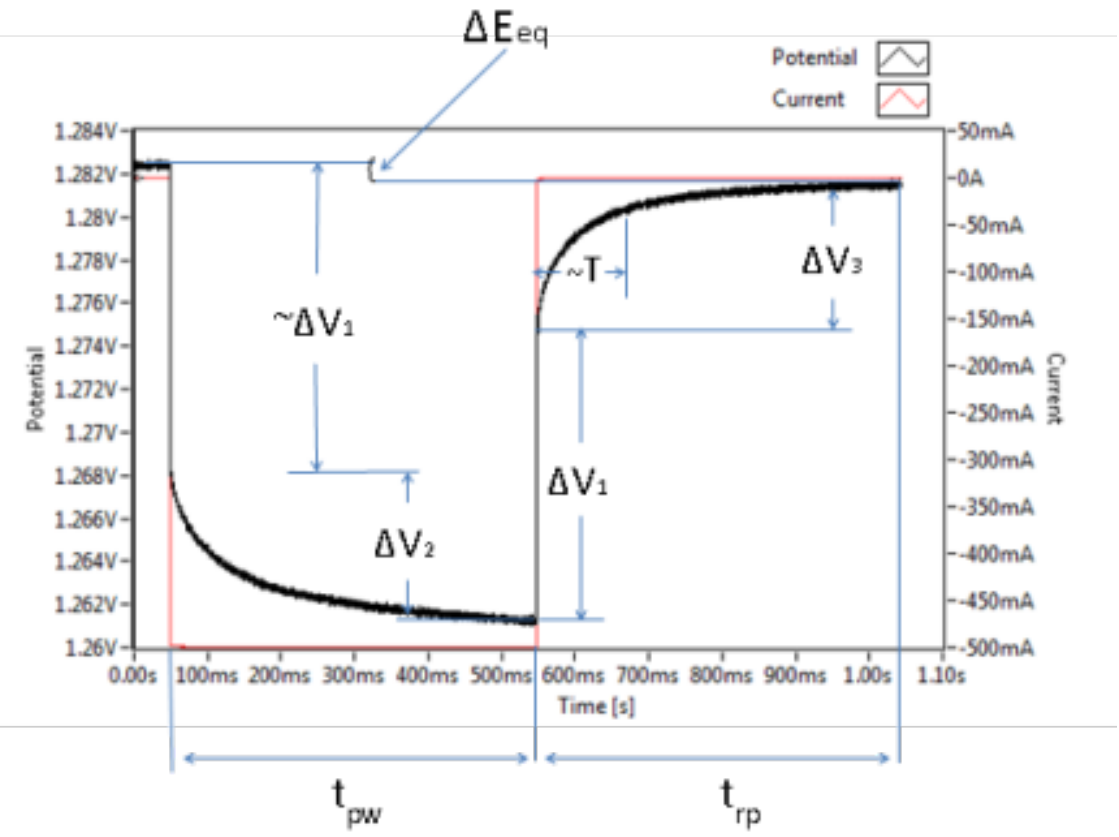

Figure 82. Parameter definition in SIS for battery and fuel cell systems.

In a typical experiment the user have to select the pulse properties based on the following guidelines.

tpw $>5(T)$

$\operatorname{trp}>5(\mathrm{~T})$

Where: $T=R_{c t} C_{d}$ is the time for which the amplitude of the potential changes $e^{-1}$ times.

When the above theoretical approach is applied to the real system the EZware will calculate the cell parameters as demonstrated in Figure 83. 


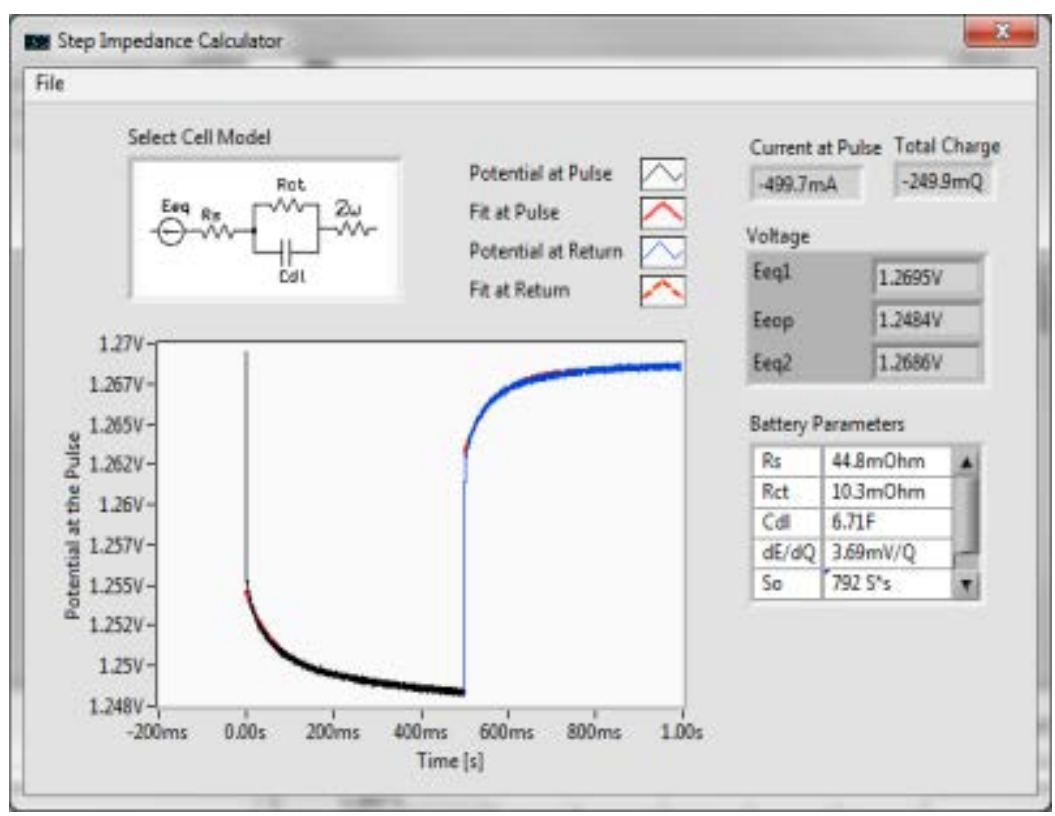

Figure 83. SIS analysis on a NiMeH single cell battery. $I_{p}=-0.5 A$.

\section{Case 2.}

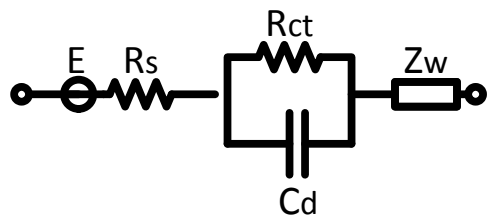

Figure 84. Modified Randles cell for battery electrode. E=const: battery voltage, Rs: solution resistance, Cd: double layer capacitance, Rct: charge transfer resistance, Zw: Warburg impedance (i.e., diffusion phenomenon)

This analysis if for batteries and fuel cells that do not change the $E$ during the course of the test. Assuming the $E$ is constant one can rewrite the differential equation of the system in the following manner:

$R_{c t} C_{d} \frac{d V}{d t}+V=I\left(R_{s}+R_{c t}\right)+R_{s} R_{c t} C_{d} \frac{d I}{d t}+E+Z_{w}(t)$

Where Warburg impedance is again approximated with: $Z_{w}(t)=\frac{1}{s_{0}} I t$

Then the differential equation can be rewritten: 
$R_{c t} C_{d} \frac{d V}{d t}+V=I\left(R_{s}+R_{c t}\right)+R_{s} R_{c t} C_{d} \frac{d I}{d t}+E+\frac{1}{S_{0}} I t$

The solution of the differential equation is:

$$
\begin{cases}V(t)=\left[i_{p} R_{s}+i_{p} R_{c t}\left(1-\frac{C_{d}}{S_{0}}\right)+E_{e q 1}\right]+\frac{i_{p}}{S_{0}} t+i_{p} R_{c t}\left(\frac{C_{d}}{S_{0}}-1\right) e^{-\frac{t}{R_{c t} C_{d}}} & I=i_{p} \\ \text { and } & I=0 \\ V(t)=E+\left(V_{0}-E-i_{p} R_{s}-\frac{i_{p} i_{p w}}{S_{0}}\right) e^{-\frac{t}{R_{c t} C_{d}}} & I=0\end{cases}
$$

In this case the parameters in system above are extracted in similar manner as in Case 1.

Circuits used for modeling other type of electrochemical cells.

Currently NuVant Systems is working to implement the Step Impedance Spectroscopy to systems that can be approximated by the following equivalent circuits:

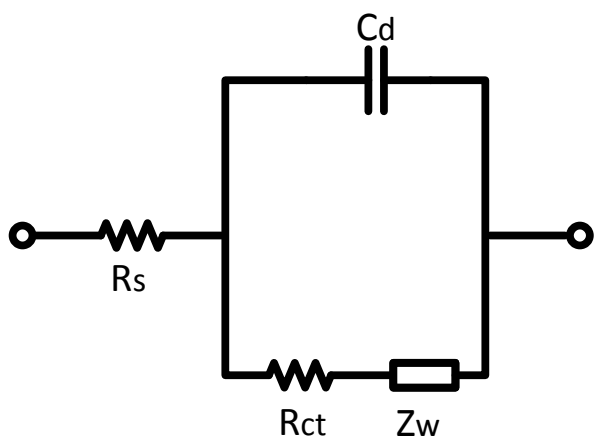

Figure 85. A standard parallel-connected resistance-capacitance circuit with the addition of Zw:

Warburg impedance 


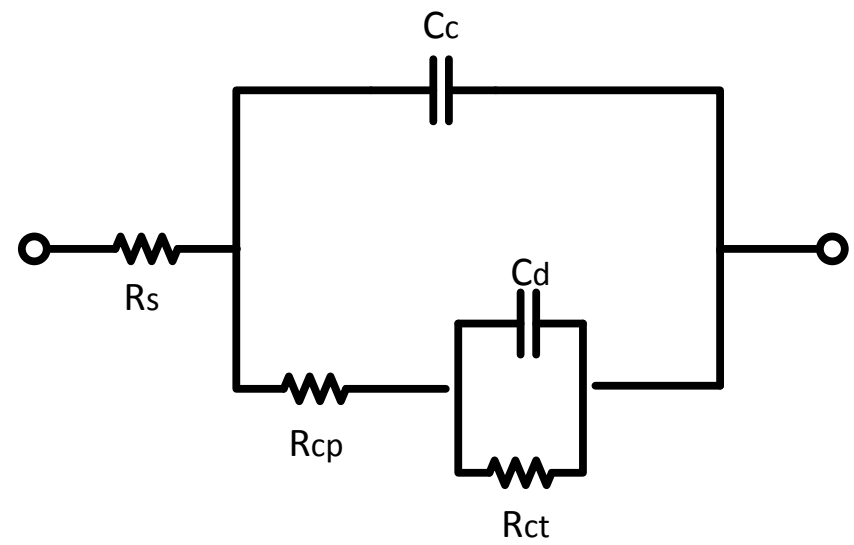

Figure 86. Electrical network analog for electrochemical impedance spectroscopy of a coated metal surface. Rs: solution resistance; Rcp: porous coating resistance; Rct: charge transfer resistance; Cc: coating capacitance; Cd: pseudo-double-layer capacitance.

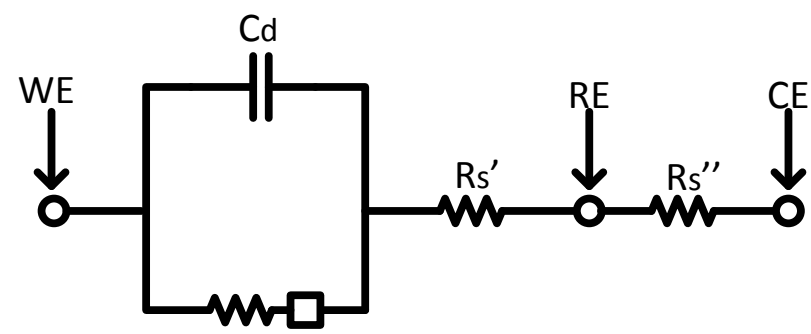

Rct ZD

Figure 87. . Electrical equivalent a single electrode-solution interface connected to a potentiostat. ZD : mass-transport element RE: reference electrode, CE: counter electrode, WE: working electrode

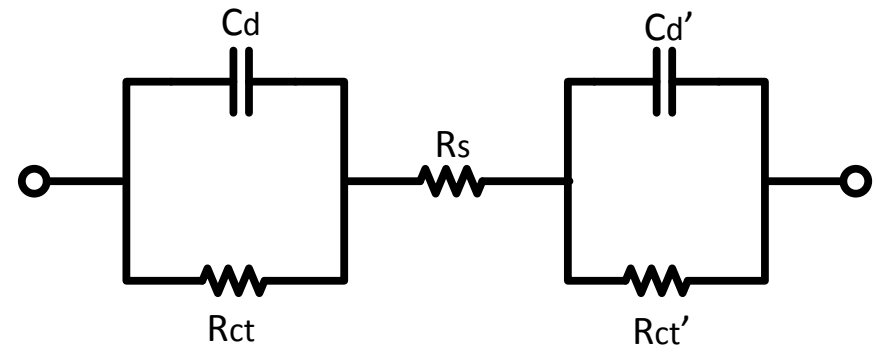

Figure 88. Electrical equivalent for a two-electrode system 


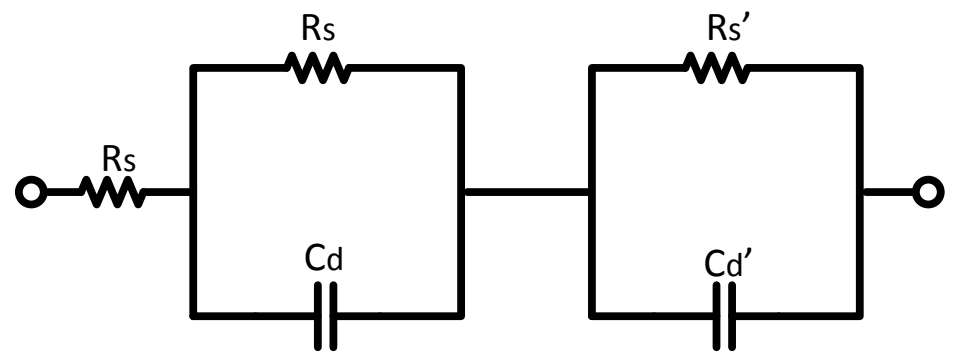

Figure 88. Schematic diagram of the 2RC element circuit, for which impedance was calculated based on synthetic data of the time-domain transient, being a response to current pulse excitation

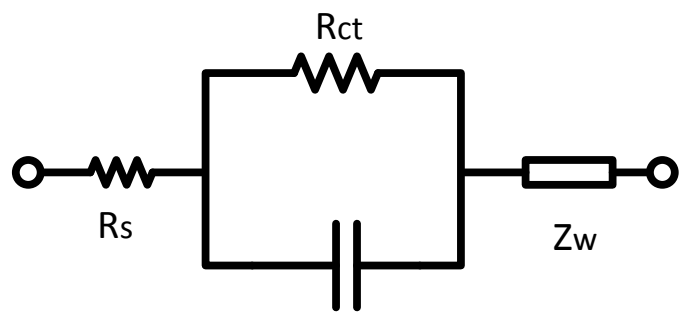

$\mathrm{Cd}$

Figure 89. Equivalent electrical circuit diagram of a $\mathrm{NiMH}$ battery representing the overpotential during charging and discharging without modeling of the equilibrium potential

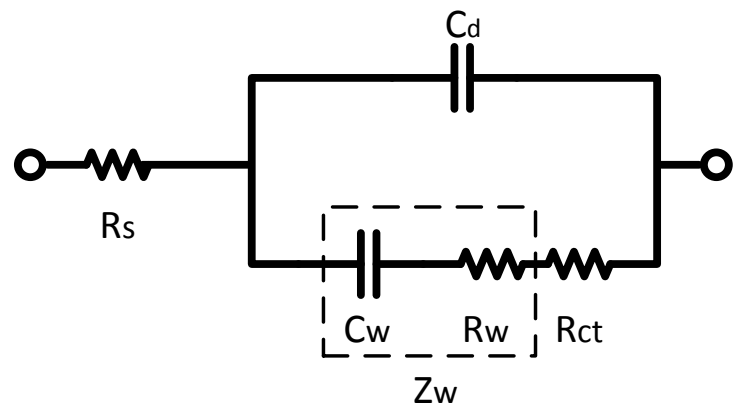

Figure 90. Where the charge transfer resistance Rct manifests the kinetics of heterogeneous charge transfer and the component of the Warburg impedance, Rw and Cw manifests diffusional mass transfer 
While the Step Impedance Spectroscopy toolbox was not fully developed and tested for the IFDL DMFC NuVant is working actively to test it and prove its usefulness for diagnosing potential problems inside of the fuel cell such as flooding, electrode delamination, etc.

\section{Final Conclusions}

Over the course of the project NuVant was able to develop a semi-passive DMFC capable of operating with highly concentrated methanol which delivered high GFED. The increase in GFED over the course of the project is shown in figure 91.

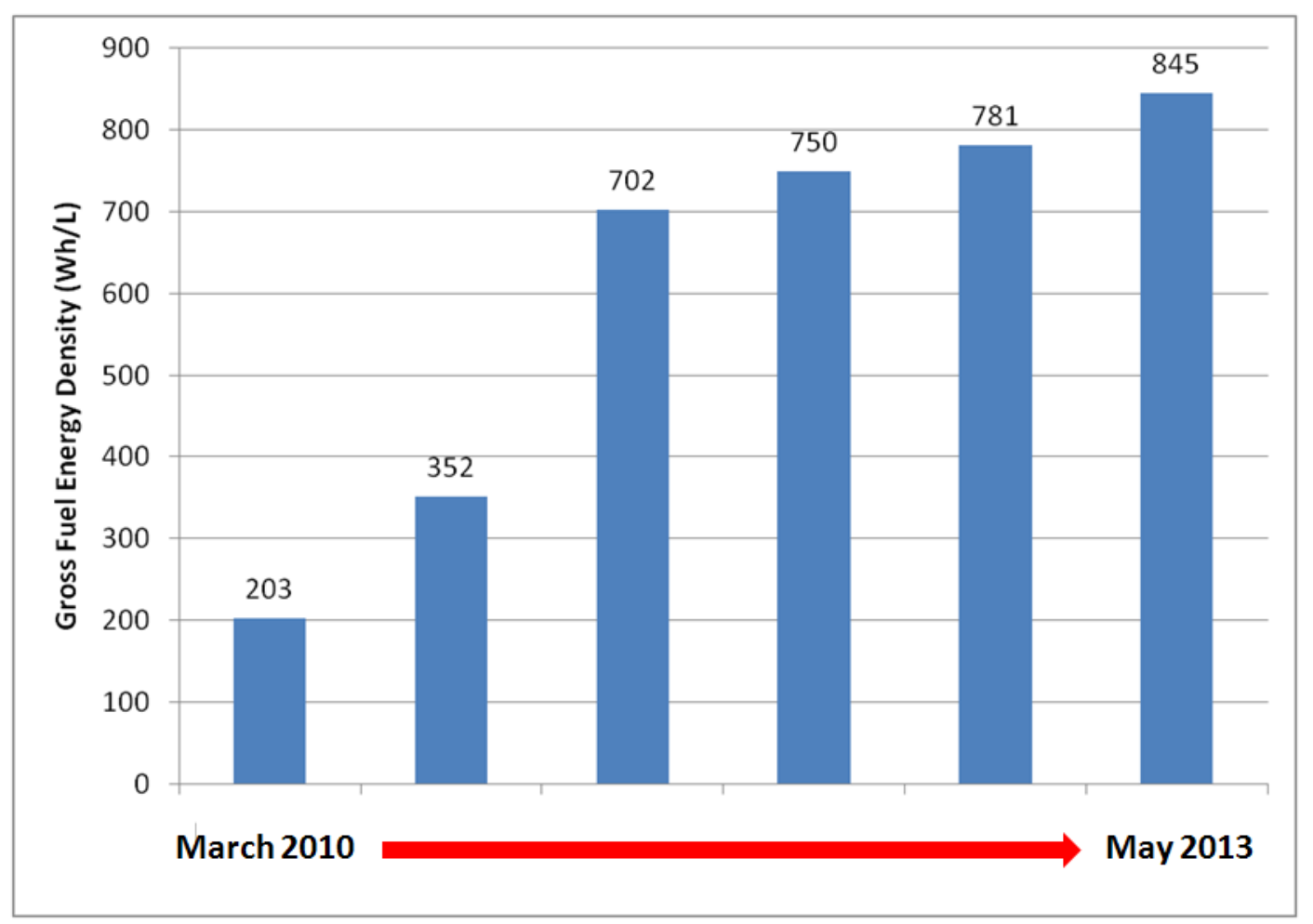

Figure 91. The increase in GFED over the course of the project

This final value of the GFED is at the same level or better than that reported by established DMFC system manufacturers. Values of the GFED disclosed by established manufacturers are sparse but a few of them are shown in figure 92. 


\begin{tabular}{|l|c|}
\hline Fuel Cell Project/System & GFED (W $\left.{ }^{*} \mathbf{h} / \mathbf{L}\right)$ \\
\hline NuVant IFDL DMFC ${ }^{1}$ & 845 \\
\hline SEC JENNY2 & 800 \\
\hline EFOY 1600-M52 $^{2}$ & 577 \\
\hline EFOY 1600-M102 $^{2}$ & 650 \\
\hline EFOY 1600-M28 $^{2}$ & 607 \\
\hline Oorja Pac & \\
\hline UltraCell XX252 & 909 \\
\hline Passive DMFC Single Cell3 & 692 \\
\hline
\end{tabular}

1. Best performance $25 \mathrm{~cm}^{2}$ single cell

2. DMFC power system data; $S$. Narayan and T. Valdez, ECS Interface Winter 2008, p. 40

3. Xioaming Ren, et al. Patent \# US 7541109B2, Jun 2, 2009

Based on the results over the course of this project we can conclude the following:

$>$ GFED in the same range as the established DMFC manufacturers

$>$ Research-grade stack was successfully operated

$>$ Small manufacturing line to produce GDLs at custom hydrophobicity levels was designed and is successfully operating 